\title{
Essays on bounded rationality in epistemic game theory
}

Citation for published version (APA):

Mounir, A. (2016). Essays on bounded rationality in epistemic game theory. [Doctoral Thesis, Maastricht University]. Datawyse / Universitaire Pers Maastricht. https://doi.org/10.26481/dis.20161220am

Document status and date:

Published: 01/01/2016

DOI:

10.26481/dis.20161220am

Document Version:

Publisher's PDF, also known as Version of record

\section{Please check the document version of this publication:}

- A submitted manuscript is the version of the article upon submission and before peer-review. There can be important differences between the submitted version and the official published version of record.

People interested in the research are advised to contact the author for the final version of the publication, or visit the DOI to the publisher's website.

- The final author version and the galley proof are versions of the publication after peer review.

- The final published version features the final layout of the paper including the volume, issue and page numbers.

Link to publication

\footnotetext{
General rights rights.

- You may freely distribute the URL identifying the publication in the public portal. please follow below link for the End User Agreement:

www.umlib.nl/taverne-license

Take down policy

If you believe that this document breaches copyright please contact us at:

repository@maastrichtuniversity.nl

providing details and we will investigate your claim.
}

Copyright and moral rights for the publications made accessible in the public portal are retained by the authors and/or other copyright owners and it is a condition of accessing publications that users recognise and abide by the legal requirements associated with these

- Users may download and print one copy of any publication from the public portal for the purpose of private study or research.

- You may not further distribute the material or use it for any profit-making activity or commercial gain

If the publication is distributed under the terms of Article $25 \mathrm{fa}$ of the Dutch Copyright Act, indicated by the "Taverne" license above, 


\section{EsSAYs ON Bounded RATIONALITY IN Epistemic Game Theory}

Angie Mounir 
Copyright Angie Mounir, 2016 ISBN $978 i 9461596499$

Print Datawyse - Universitaire Pers Maastricht 


\section{EsSAYS ON Bounded RATIONALITY IN EPISTEMic Game Theory}

\section{PROEFSCHRIFT}

ter verkrijging van de graad van doctor

aan de Universiteit Maastricht, op gezag van Rector Magnificus, Prof. dr. Rianne M. Letschert, voglens het besluit van het College van Decanen, in het openbaar te verdedigen op dinsdag 20 december 2016 om 14:00

door

Angie Mounir 


\section{Promotor}

Prof. dr. Hans Peters

\section{Co-Promotores}

Dr. Andrés Perea

Dr. Elias Tsakas

\section{Beoordelingscommissie}

Prof. dr. Jean-Jacques Herings (voorzitter)

Prof. dr. Gerhard Weiss

Dr. Christian Bach (University of Liverpool)

Dr. Conrad Heilmann (Erasmus University Rotterdam)

Dit onderzoek werd financieel mogelijk gemaakt door de Graduate School of Business and Economics (GSBE). 
To my late grandmother Etidal 



\section{Acknowledgment}

"It is the road that teaches us the best way to get there, and the road enriches us as we walk its length."

— Paulo Coelho, The Pilgrimage

Starting in 2011, I never thought I would compare my years as a PhD student to a long hiking trip. Yet the resemblance turned out to be uncanny, from the exciting highs to the disappointing lows and all the way through the daily nitty-gritty. Starts are always exciting intertwined with a desire to make oneself proud. The first couple of times you are out of breath are always the hardest, whether it is from climbing a hill with a heavy bag or from wondering if you will ever manage to get some proof right. The highs are the best, like when you break your record of kilometers walked or when you show that your ideas work. Both journeys require plenty of patience and persistence, if they are to be completed. Most importantly though both journeys are made especially worthy by the people they are shared with. I therefore would like to take the following lines to thank those whom I have had the pleasure of sharing my $\mathrm{PhD}$ journey with.

I was lucky to have two great supervisors. Andrés and Elias, thank you for your guidance and support throughout the whole process with all its ups and downs. I am especially grateful for all the discussions we had and the thorough feedback I got from you on my work. I am also really grateful for the freedom you allowed me in finding and pursuing my interests and the support you gave me while doing so. My promotor Hans, even though we did not work closely on research, you were always ready to answer whatever questions I had. Current and former secretaries of the KE department: Karin, Yolanda and Haydeé thank you for your dedication and professionalism and for often going out of your way to fix a problem or answer a question. I would also like to thank the members of the assessment committee for taking the time to read my work and for the thorough and enriching feedback.

I cannot think of my $\mathrm{PhD}$ time and not remember ladies' night with Nadine and Elnaz: thank you girls for the fun evenings and the delicious meals. To game theory dinners with Anna and Chris I owe many interesting discussions about research and everything else. My biweekly lunches with Salomé to exchange Dutch-Arabic writing skills were a lot of fun, although we never actually got to the writing part. My dear friend and semi-flatmate Maria and my childhood 
friend Amira, thank you both for being there in so many ways. My time at Maastricht University was further enriched by the presence of many friends and colleagues: Sylvia, Gergely, Martijn, Aidas, Christine, Burak, Ingrid, Birol, Vincent, Oksana, Jan, my officemates Marc and Carlos, Ahmad, Kouma, Cigdem, Abhinaba, Murat and many others.

Thanks to Christian Bach for his often contagious enthusiasm about research in general and epistemic game theory in particular; and to János Flesch for many interesting talks over coffee and during our occasional walks home. Teaching can be fun and can be frustrating at the same time, but teaching with Alex Grigoriev ensures the fun is maximized and the frustrations are minimized, subject to the constraints of course. Because the longest mile is the last mile home, thanks are also due to my KIO colleagues at CBS Heerlen for cheering me to the finish line.

Last but not least, thanks to my family: my mother Annette, brother Ibrahim and sister Sarah for constant long-distance encouragement and support on all fronts inside and outside my $\mathrm{PhD}$. I am also very grateful to my family away from home: Lillianne, Bert, Dirk, Elly, Pim and Eva. Finally, thanks to Michel for taking up an interest in game theory just for me, for voluntarily reading through my cryptic sketches of ideas scribbled on the kitchen wall, for making me smile when most needed and many times for simply being there. 


\section{Contents}

Acknowledgements $\quad$ v

1 Introduction 1

2 Common Belief in Approximate Rationality 7

2.1 Introduction . . . . . . . . . . . . . . . . . . 7

2.2 Common Belief in $F$-Rationality . . . . . . . . . . . . . . . . . . . . 10

2.3 Iterated Elimination of $F$-Dominated Beliefs ～. . . . . . . . . . . . . . . . 13

2.3.1 The Recursive Procedure . . . . . . . . . . . . . . . . . . . . . . 13

2.3.2 Practical Implementation Matters . . . . . . . . . . . . . . . . 16

2.4 Special Cases . . . . . . . . . . . . . . . . . . . . . . 17

2.4.1 Common Belief in $\varepsilon$-Rationality . . . . . . . . . . . . . . . 17

2.4.2 Common Belief in $p$-Belief in Rationality . . . . . . . . . . . . . . . 18

2.5 An Example: The Traveler's Dilemma . . . . . . . . . . . . . . . . 20

2.5.1 Illustrating Examples of the Recursive Procedure . . . . . . . . . . . 20

2.5.2 CBFR in the Traveler's Dilemma . . . . . . . . . . . . . . 23

2.6 Discussion and Conclusion . . . . . . . . . . . . . . . . . 25

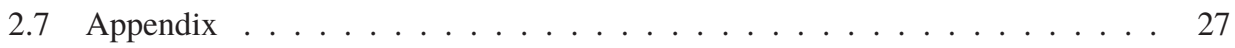

3 Common Strong Belief in Approximate Rationality 35

3.1 Introduction . . . . . . . . . . . . . . 35

3.2 Common Strong Belief in F-Rationality . . . . . . . . . . . . . . . . . 37

3.2.1 Notation and Basic Model . . . . . . . . . . . . . . . . . . . 37

3.2.2 Strong Belief in $F$-Rationality . . . . . . . . . . . . . . . . . 39

3.2.3 Common Strong Belief in $F$-Rationality . . . . . . . . . . . . . . . . . 40

3.2 .4 An Example . . . . . . . . . . . . . . . . . . . . . . 42

3.3 Iterated Elimination of $F$-Dominated Conditional Beliefs $\ldots \ldots \ldots$

3.3 .1 The Procedure . . . . . . . . . . . . . . . . . . . 44

3.3.2 Practical Matters of Implementation . . . . . . . . . . . . . . . . . . . . 46

3.3.3 An Example: The Centipede Game . . . . . . . . . . . . . . . . . . . 48

3.4 Discussion and Conclusion . . . . . . . . . . . . . . . 51

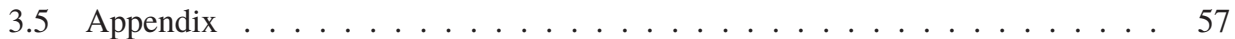

4 Reasoning Towards Common Belief in Reference Dependent Rationality 69

4.1 Introduction . . . . . . . . . . . . . . . . . . . . . . . 69

4.2 Common Belief in Reference Dependent-Rationality . . . . . . . . . . . . 73

4.2.1 Reference-Dependent Utility . . . . . . . . . . . . . . . . . . 73

4.2.2 The Epistemic Model . . . . . . . . . . . . . . . . . . . . 74

4.2.3 The Epistemic Model: An Example . . . . . . . . . . . . . . . . 76 
4.3 Characterization of Reference Dependent Rational Choices . . . . . . . . . . . 78

4.3.1 Characterization Result . . . . . . . . . . . . . . . . . . 78

4.3.2 Illustrating Examples of Lemma $4.1 \ldots \ldots$. . . . . . . . . . . . . . 79

4.4 Iterated Elimination of RD-dominated Choices (IERDC) . . . . . . . . . . 81

4.4.1 The Algorithm: An Example . . . . . . . . . . . . . . . . . . 84

4.5 The Algorithm as a Process of Deliberation . . . . . . . . . . . . . . 85

4.5.1 IERDC as a Categorization Procedure . . . . . . . . . . . 86

4.5.2 Reasoning Towards CBRDR . . . . . . . . . . . . . . . . 87

4.5.3 How Do Players Form Expectations of their own Choices? . . . . . . . 88

4.6 Discussion and Conclusion . . . . . . . . . . . . . . . . . 89

4.6.1 The Case of Gains Receiving Positive Weight . . . . . . . . . . . . . 89

4.6.2 Links to the Kôszegi and Rabin (2006) Utility Function . . . . . . . . . 90

4.6.3 Multidimensional Utility . . . . . . . . . . . . . . . . . . . . . . 91

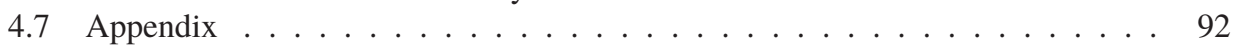

$\begin{array}{ll}\text { Bibliography } & 101\end{array}$

$\begin{array}{ll}\text { Valorisation } & 105\end{array}$

$\begin{array}{lr}\text { Nederlandse Samenvatting } & 109\end{array}$

$\begin{array}{ll}\text { Curriculum Vitae } & 111\end{array}$ 


\section{Chapter 1}

\section{Introduction}

Game theory is concerned with modeling the decision making of players in situations of strategic interaction, i.e. where a player's payoff is dependent not only on his own choice but also on what his opponents would do. Classical non-cooperative game theory focuses in that context on developing solution concepts that characterize the equilibrium outcome of the strategic interaction or game played by rational players. Epistemic game theory, on the other hand, takes a different approach by placing the focus on the reasoning process and the resulting choice behavior of individual players rather than the equilibrium outcome of the game. Thus reasoning of players about the strategic uncertainties they face in an attempt to make the "best possible" choices is at the heart of epistemic game theory.

Uncertainties in games could pertain to every aspect of the environment in which the players make their choices. The basic space of uncertainty facing any player contains the choices his opponents could make as well as any parameter of the model unknown to that player. Consider a game of incomplete information where each player's utility function is his private information. Then the basic space of uncertainty of any player would consist of all possible combinations of opponents' choice-combinations and utility functions. One state of nature therefore represents one potential combination of opponents' choices and utility functions. The basic space of uncertainty is often referred to as states of nature (Pacuit and Roy, 2016). In addition to his basic space of uncertainty, the player also faces uncertainties regarding the beliefs his opponents hold about their basic uncertainties, the beliefs these opponents hold about their opponents' beliefs, and so on. Therefore, the reasoning process of a player involves the formation of that player's beliefs about the basic uncertainty he faces, his beliefs about the beliefs held by his opponents about their basic uncertainty, his beliefs about his opponents' beliefs about their opponents' beliefs, and so on.

The process of reasoning about the game players face and about their opponents' beliefs results in the formation of belief hierarchies. Characterized by an infinite number of layers, belief hierarchies are not only crucial objects in epistemic game theory but also very complex ones. 
One way to encode these hierarchies of beliefs is using the type space model due to Harsanyi (1967-68). First designed to encode players' beliefs about parameters of the game that are private information of their opponents in games of incomplete information, the use of the type space model was later extended to model players' beliefs about any interactive uncertainty in the game as well as their beliefs about their opponents' respective beliefs. In a type space model, each player is assigned a set of types each of which induces a belief hierarchy this player might hold. Every epistemic type of some player is associated with a belief about combinations of the basic space of uncertainty facing that player and his opponents' types.

In epistemic game theory concepts restrictions are placed on players' possible belief hierarchies in order to distinguish those that are consistent with the concept of interest from the ones that are not. Restrictions imposed on belief hierarchies in epistemic concepts most commonly assume players commonly believe in a certain way of reasoning or method of choice (see for example, Lewis (1969) and Aumann (1976)). The most prominent example of such concepts is Common Belief in Rationality, where each player believes his opponents choose rationally, believes his opponents believe their opponents choose rationally, believes his opponents believe their opponents believe they choose rationally, and so on. The definition of rationality at the heart of common belief in rationality assumes players maximize their subjective expected utility given their beliefs about the choices and beliefs of their opponents. Common belief in rationality is considered to be the benchmark to which other epistemic concepts in the literature are compared. Since the focus of this thesis is bounded rationality, it is without a doubt that Common Belief in Rationality will be playing a similar role throughout the following chapters.

The concept of bounded rationality finds its origin in the historical development of behavioral economics. Perfect rationality axioms introduced by von Neumann and Morgenstern (1944) and Savage (1954) were interpreted as capturing the general aspects of rationality. The axioms characterized how players would want to behave even if they occasionally failed to live up to their desired behavior in practice. Nevertheless, the theories were not separate from reality as they were presumed to be generalizations of empirical observations supporting them. Notably, proponents often stressed that the perfect rationality axioms do not by any means lend themselves to empirical testing despite them being generalization of empirical observations. Growing dissatisfaction with the dual nature of the interpretation of the axioms along with subsequent developments in the field of psychology led to a broad distinction between two definitions of rationality; normative versus descriptive. The former interpreted the axioms as characterizing rationality in a philosophical and abstract manner, while the latter pertained to patterns of actual behavior observed in experiments. The normative definition of rationality remained to be perceived as the optimal way of behavior players aspired to follow (Heukelom, 2014). 
The work of psychologists Kahneman and Tversky is considered a major development in the evolution of behavioral economics. Their work was part of a wider range of attempts in the 1970s to account for both the desire to maintain the axioms and the need to account for the growing evidence against them. Kahneman and Tversky maintained the desirability of the axioms as the norm of behavior and presumed people would want to change their behavior once their deviation from the norm is pointed out to them. However, they also acknowledged that deviations from that rational norm occur more systematically than previously thought. Players try to make the best decisions possible and systematically fall short thereof because they rely on heuristics and make cognitive errors. Thus rationality axioms were preserved as the rational norm while the theoretical formulation of deviations became the measurement framework fitting observed results (Kahneman, 2011 and Heukelom, 2014).

Thus deviations from subjective expected utility maximization could be perceived as errors reflecting limitations on the cognitive abilities of players or interference of emotions in the reasoning process about a given strategic situation. However, an alternative approach considers non-expected utility preferences as alternative models of rational behavior (take for example the interpretation of Loomes and Sugden (1982) of their model of regret-rejoice utility). According to this second approach, violations of perfect rationality occur in practice because the latter does not necessarily reflect how people reason and choose. Thus even if presented with elaborate arguments explaining their deviation from the expected utility maximizing choice(s), players might still not want to change their choice. Furthermore, proponents of this approach would argue that realizing that one experiences certain feelings such as regret, disappointment, or attachment to the status quo and taking that into account in the process of belief formation and choice cannot be described as anything but rational.

Epistemic models of bounded rationality in this thesis can be broadly classified in two parts. Part I, including Chapters 2 and 3, assumes players to be expected utility maximizers but allows for the possibility that players make mistakes in optimizing their choices and/or believe their opponents might err. Players are thus approximately rational in the sense that they (try to) come very close to rational choice represented by expected utility maximization. Chapter 2 defines Common Belief in Approximate Rationality in static games, which is then extended to dynamic games in Chapter 3. Hence, part I can be said to maintain the normative desirability of perfect rationality as a model of behavior players aspire to follow as close as possible, with players' bounded rationality manifesting itself in the possibility of making mistakes.

Part II, or Chapter 4, examines an expectation-based reference-dependent utility model. In this case, the category to which this model can be assigned in terms of how the perfect rationality 
approach is viewed is less obvious. On the one hand, behavior represented by referencedependent utility can be thought of as an irrational violation of rational choice, one that players would want to take back were they to be made aware of it. On the other hand, viewing players as being aware of the status quo effect created by their initial expectations and consciously taking that into account in forming their beliefs and making their choice could be interpreted as an alternative form of rational choice. I personally choose for the second interpretation. One manifestation of such choice is highlighted further below in this introduction.

In each of the three main chapters of this thesis the specific concept of rationality is embedded within an epistemic model where the appropriate restrictions are defined to identify those epistemic types that express common belief in that specific concept of rationality. Note that type spaces in the literature are often taken to be complete (Brandenburger 2003), i.e. to include all possible belief hierarchies to ensure generality of the results. However, throughout this thesis only finite or countably infinite type spaces are adopted. This choice, following an existing approach in the literature (see for example Perea (2012)), is motivated by a desire to keep the models simple and intuitive and by a belief that most concepts can be adequately represented without any loss of generality by finite or countably infinite models. Throughout the thesis, the game under consideration is viewed and analyzed from the perspective of one player, a representative player. Each chapter of this thesis can be read independently and has its own introduction and discussion section, with notation defined within each chapter.

Chapter 2 substitutes the standard rationality assumption with $\varepsilon$-rationality in normal form games. Players are assumed to believe their opponents might be $\varepsilon$-rational, i.e. willing to settle for a suboptimal choice, and so give up an amount $\varepsilon$ of expected utility, in response to the belief they hold. However, each player's $\varepsilon$ is private information. For every player $i$ and every opponents' degree of rationality $\varepsilon$, player $i$ is required to attach at least probability $F_{i}(\varepsilon)$ to his opponent being $\varepsilon$-rational, where the functions $F_{i}$ are assumed to be common knowledge. This event is referred to as belief in $F$-rationality. The notion of Common Belief in $F$-Rationality is then introduced as an approximate rationality counterpart of the established Common Belief in Rationality.

By applying the model of Chapter 2 to an $n$-price Traveler's Dilemma game, it is shown that by assigning only a relatively small probability to the event of his opponent making an error, it might be optimal for a player to choose anything up to the second highest price. This is at odds with the predictions of Common Belief in Rationality in the Traveler's Dilemma where rational players can only be expected to choose the lowest possible price, can only believe their opponents would choose the lowest possible price and so on. A minimum threshold for the probability that a player needs to assign to the event of his opponent making a mistake is derived 
and is shown to be a decreasing function of $n$. The model introduced in Chapter 2 is extended in Chapter 3 to dynamic games where the approximate rationality counterpart of Common Strong Belief in Rationality (Battigalli and Siniscalchi 2002) is defined.

While already extensively studied in the literature, reference-dependent utility has never been considered within an epistemic framework. Instead it has always been studied within an equilibrium framework requiring players to have correct beliefs about their opponents choices and beliefs. Furthermore, the extent of players' sensitivity to losses, represented by the loss-aversion coefficient, has always been assumed to be common knowledge. The model examined in Chapter 4 formalizes the concept of Common Belief in Reference-Dependent Rationality where the players' extent of sensitivity to losses are assumed to be their private information. This concept is also shown to be equivalent to Common Belief in Ordinal Preferences over Pure Outcomes and rationality given some von Neumann-Morgenstern utility function consistent with player's ordinal preferences (Börgers 1993; and Duggan and Le Breton, 2014).

For each of the models introduced, a computational procedure characterizing that respective model is defined and shown to work. In the case of Chapter 4, the procedure is also reinterpreted as a process of deliberation of finite steps that players follow to reach the end goal of forming their beliefs. It is worth noting that such reinterpretation is not standard in the literature, as most epistemic concepts only specify the outcome in terms of beliefs of a reasoning process with certain characteristics and do not indicate how these beliefs are formed. Two important contributions in which iterative procedures are interpreted as processes of reasoning are those of Cubitt and Sugden (2011) and Perea (2015). Such reinterpretation of the iterative procedure serves as a confirmation of how I believe the model of Chapter 4 should be viewed relative to the perfect rationality approach. 



\section{Chapter 2}

\section{Common Belief in Approximate Rationality}

\subsection{Introduction 1}

Rationality of players in situations of strategic interaction has been a crucial axiom upon which the vast majority of game theoretic concepts is based. A player is rational if he only plays optimal choices, which are those that maximize his expected utility given his beliefs about opponents' choices. Specifying the set of choices any rational player can make in a normal form game has therefore been the central question many existing solution concepts attempted to answer. Rationalizability, epistemically characterized by rationality and Common Belief in Rationality (CBR) (Pearce, 1984; Bernheim, 1984; Brandenburger and Dekel, 1987; Tan and Werlang, 1988), is a crucial one of such concepts. Under rationality and CBR, all players are rational, believe in their opponents' rationality, and so on. Choices that can be made under CBR are those that survive Iterated Elimination of Strictly Dominated Choices (IESDC).

Despite its solid epistemic foundations, experimental findings in certain well-known games came at odds with rationality and CBR. Examples of such games include, the Traveler's Dilemma and Guess 2/3 of the Average (Nagel, 1995; Becker, Carter, and Naeve, 2005). This discrepancy between experimental outcomes and theoretical ones triggered the need to develop new theoretical models of reasoning that provide better theoretical foundations for observed experimental behaviour in these games. One such aspect highlighted in experiments is that players may err and/ or believe that their opponents might make mistakes.

One way to introduce such mistakes is by replacing the standard notion of rationality with $\varepsilon$-rationality, originally introduced by Radner (1980). The basic underlying idea is that players may settle for suboptimal choices, as long as the utility induced by these choices is sufficiently

\footnotetext{
${ }^{1}$ This chapter is a joint work with Andrés Perea and Elias Tsakas
} 
close to the utility induced by the optimal ones. Formally, a choice is $\varepsilon$-rational given a belief about the opponents' choices, whenever the expected utility of this choice (given the belief) is at most $\varepsilon$ away from the optimal expected utility (given the same belief). This idea initially attracted a lot of attention, as it allowed us to explain cooperation in finitely repeated prisoner's dilemma or in finitely repeated principal-agent games (e.g., see Radner, 1981), which is not possible using more standard solution concepts such as Nash equilibrium or Common Belief in Rationality. Still the behavioral foundations of $\varepsilon$-rationality remained in the background.

The recent surge of behavioral economics, as well as work in other disciplines, have provided basis for assuming that players may sometimes be $\varepsilon$-rational. For instance, in their seminal work, March and Simon (1958) introduced the notion of "satisficing" which replaced the usual concept of "optimizing". In our context, this would mean that players may settle for suboptimal choices as long as these are sufficiently close to the optimal one, thus making them satisfactory. Another example is the well-known Weber-Fechner-Stevens set of laws from psychophysics, according to which people may fail to perceive (small) differences when they compare two choices, thus implying that the size of $\varepsilon$ represents the player's cognitive constraints. In either case, Baye and Morgan (2004) provide empirical evidence supporting $\varepsilon$-rationality when compared to standard rationality.

The notion of $\varepsilon$-rationality originally appeared in the definition of Radner's $\varepsilon$-equilibrium concept, while more recently, Dekel, Fudenberg and Morris (2006) introduced the concept of $\varepsilon$-interim correlated rationalizability. In both $\varepsilon$-equilibrium and $\varepsilon$-interim correlated rationalizability, the value of each player's $\varepsilon$ was assumed to be transparent with players having common belief therein. This is relaxed in our model, as players are assumed to be $\varepsilon$-rational with the value of each player's $\varepsilon$ being his private information. Thus players in our model not only account for the possibility of potential mistakes on their opponents' part, but also perceive the exact margin of error their opponents might have as uncertain. Therefore, players form beliefs about the extent of their opponents' potential errors. In an attempt to restrict such uncertainty, players adopt a certain lower bound to the beliefs they could form about their opponents' error margins. Such lower bound is captured by the commonly believed weakly increasing functions $F_{i}:[0, \infty) \rightarrow[0,1]$. For every player $i$, the function $F_{i}$ assigns a minimum bound to the probability that player $i$ would assign to each potential level of the opponents' $\varepsilon$.

Thus the function $F_{i}$ does not specify the exact belief player $i$ assigns to each of his opponents' possible extents of rationality, but serves only as a lower bound for such beliefs. The lower the extent of irrationality $\varepsilon$, the lower the minimum bound on probability $F_{i}(\varepsilon)$ each player must assign to the event of his opponents having a margin of error of at most $\varepsilon$. For every possible extent of irrationality $\varepsilon$ of player $i$, there is a set of choices that could be $\varepsilon$-rationally 
made by player $i$ given some belief about his opponents' choices. The uncertainty about the opponents' extent of irrationality can be translated into restrictions on the probabilities that could be assigned to different sets of opponents' choice combinations. This translation of uncertainty into restrictions on probabilities assigned to sets of opponents' choice combinations, plays a crucial role throughout the paper.

For every given level $\varepsilon$ of the opponents' error margin, a lower bound of $F_{i}$ means player $i$ assigns at least probability $F_{i}(\varepsilon)$ to the event of his opponents' willingness to give up an amount of $\varepsilon$ or less in terms of expected utility. We refer to this event as belief in $F$-rationality. Establishing common belief among all players in that event, this paper introduces Common Belief in $F$-Rationality. Notably, this framework captures the intuitive uncertainty players might have about the extent to which their opponents might deviate from their optimal choices. Moreover, adopting $F$-rationality offers a generalization to some of the existing concepts in the literature such as $\varepsilon$ rationality and, of course, common belief in rationality. It also helps linking some existing concepts in the literature such as Common Belief in $\varepsilon$ Rationality and Common $p$-Belief in Rationality.

Models and concepts in the literature that are in the same spirit as ours, include Rosenthal's $t$-Solution (Rosenthal, 1989), Quantal Response Equilibria (McKelvey and Palfrey, 1995) and Utility Proportional Beliefs (Bach and Perea, 2014). All of these models recognize that players might assign positive probabilities to opponents' suboptimal choices, none however explicitly defines the underlying reason to be the presence of a potential error margin. $\mathrm{CB} F \mathrm{R}$, just like all of the three aforementioned models, allows players to assign positive probabilities to suboptimal choices of their opponents'. However, CBFR differs in that positive probabilities need not be assigned to all opponents' choices. Moreover, in all three of the models mentioned, better choices receive higher probabilities, and in case of utility proportional beliefs and Rosenthal's $t$-Solution these probabilities are proportional to the utility generated by these choices ${ }^{2}$. In our case, restrictions are imposed on the probabilities assigned to groups of choices rather than on the probabilities assigned to each individual choice. Furthermore, unlike CBFR, both Rosenthal's $t$-Solution and Quantal Response Equilibria are equilibrium concepts.

After the concept is introduced, the recursive procedure of Iterated Elimination of $F$-Dominated Beliefs (IEFB) is developed, which characterizes exactly those first-order beliefs that can be held under common belief in $F$-rationality. Furthermore, a fixed point characterization of the concept of $\mathrm{CB} F \mathrm{R}$ is provided. The paper also considers two special cases. One case is that in which a specific value of $\varepsilon$ for each player is given full probability, or Common Belief in $\varepsilon$-rationality.

${ }^{2}$ More precisely, in both utility proportional beliefs and Rosenthal's $t$-Solution, the differences in probabilities assigned to opponents' choices are proportional to the differences in utilities generated by these choices. 
The second special case is where there is common belief that each player assigns at least $p$ to the event of his opponent being 0-rational, or Common Belief in $p$-Belief in Rationality. Finally, the model is applied to the $n$-price Traveler's Dilemma game to demonstrate the potential behavioral implications of our epistemic solution concept. The paper is divided into six sections. Section 2.3 defines Common Belief in $F$-Rationality. Section 2.3 presents the recursive procedure of Iterated Elimination of $F$-Dominated Beliefs. Section 2.4 covers the two special cases. Section 2.5 uses a 3-price Traveler's Dilemma game to illustrate how the recursive procedure works and then summarizes some general results for the $n$-price game. Section 2.6 is a discussion and conclusion of the paper.

\subsection{Common Belief in $\boldsymbol{F}$-Rationality}

Consider an $n$-player finite normal form game $\Gamma=(\mathcal{I}, C, U)$, where $\mathcal{I}=\{1, \ldots, n\}$ is the finite set of players, $C=\left\{C_{1}, \ldots, C_{n}\right\}$ is an $n$-tuple of finite sets of choices and $U=\left\{U_{1}, \ldots, U_{n}\right\}$ where $u_{i}: C_{i} \times C_{-i} \rightarrow \mathfrak{R}$ is the utility function of player $i$. Let $b_{i} \in \Delta\left(C_{-i}\right)$ be a belief player $i$ holds about his opponents' choice combinations, where $\Delta\left(C_{-i}\right)$ is the set of probability distributions on $\left(C_{1} \times \ldots C_{i-1} \times C_{i+1} \times \ldots C_{n}\right)$. Expected utility ${ }^{3} u_{i}\left(c_{i}, b_{i}\right)$ of the choice $c_{i} \in C_{i}$ is then the utility of that choice given the belief $b_{i}$ player $i$ holds about his opponents' choices, i.e., $u_{i}\left(c_{i}, b_{i}\right):=$ $\sum_{c_{-i} \in C_{-i}} b_{i}\left(c_{-i}\right) \times u_{i}\left(c_{i}, c_{-i}\right)$. Also let $F_{i}$ be a weakly increasing function $F_{i}:[0, \infty) \rightarrow[0,1]$ held by player $i$ and $F=\left(F_{1}, \ldots, F_{n}\right)$. For every player $i, F_{i}$ characterizes a lower bound for the belief that player can hold about each of his opponents' potential values of $\varepsilon$.

Common Belief in $F$-Rationality implies every player $i$ believes in his opponents' $F$-rationality, believes his opponents believe in $i$ 's $F$-rationality, and so on. A formal characterization of CBFR, therefore, requires the use of infinite belief hierarchies, which can be intelligently defined within an epistemic model. Let $M$ be a finite epistemic model assigning to every player $i$ a finite set of types $T_{i}$. Every type $t_{i} \in T_{i}$ holds a belief $b_{i}\left(t_{i}\right) \in \Delta\left(C_{-i} \times T_{-i}\right)$ which is a probability distribution over the opponents' choice-type combinations.

As usual, each $t_{i} \in T_{i}$ induces an infinite belief hierarchy, with $b_{i}^{1}\left(t_{i}\right) \in \Delta\left(C_{-i}\right)$ being the first-order belief held by $t_{i}$ (See for instance Heifetz and Samet (1998) for more details on how an epistemic type induces a belief hierarchy). Moreover, let $u_{i}\left(c_{i}, t_{i}\right)$ be the expected utility of choice $c_{i}$ given the first-order belief held by type $t_{i}$, i.e., $u_{i}\left(c_{i}, t_{i}\right):=u_{i}\left(c_{i}, b_{i}^{1}\left(t_{i}\right)\right)$. A choice-type pair $\left(c_{i}, t_{i}\right)$ is then $\varepsilon$-rational if $c_{i}$ is $\varepsilon$-optimal given $b_{i}^{1}\left(t_{i}\right)$.

${ }^{3}$ Note that we are using the same notation $u_{i}$ to refer to both the utility of player $i$ and the expected utility of choice $c_{i}$ of player $i$ given belief $b_{i}$. The former has the form $u_{i}: C_{i} \times C_{-i} \rightarrow \mathfrak{R}$, while the latter is written as $u_{i}\left(c_{i}, b_{i}\right)$. 
Definition 2.1 A choice-type pair $\left(c_{i}, t_{i}\right)$ of player $i$ is $\varepsilon$-rational for some $\varepsilon \geq 0$, if for all $c_{i}^{\prime} \in C_{i}$,

$$
u_{i}\left(c_{i}, b_{i}^{1}\left(t_{i}\right)\right) \geq u_{i}\left(c_{i}^{\prime}, b_{i}^{1}\left(t_{i}\right)\right)-\varepsilon .
$$

A type $t_{i}$ believes in the opponents' $F$-rationality if for every $\varepsilon \geq 0$ it assigns at least probability $F_{i}(\varepsilon)$ to opponents' $\varepsilon$-rational choice-type combinations $\left(c_{-i}, t_{-i}\right)$. Note that player $i$ 's opponents' choice-type combination $\left(c_{-i}, t_{-i}\right)=\left(\left(c_{1}, t_{1}\right), \ldots\left(c_{i-1}, t_{i-1}\right),\left(c_{i+1}, t_{i+1}\right), \ldots\left(c_{n}, t_{n}\right)\right)$ is said to be $\varepsilon$-rational, if every individual opponent's choice combination $\left(c_{j}, t_{j}\right)$ is $\varepsilon$-rational where $j \neq i$. To formally define belief in opponents' $F$-rationality, let $R_{-i}^{\varepsilon}:=\left\{\left(c_{-i}, t_{-i}\right) \in\left(C_{-i} \times T_{-i}\right) \mid c_{-i}\right.$ is $\varepsilon$-optimal for $\left.b_{-i}^{1}\left(t_{-i}\right)\right\}$.

Definition 2.2 A type $t_{i}$ believes in the opponents' $F$-rationality if for every $\varepsilon \geq 0$,

$$
b\left(t_{i}\right)\left(R_{-i}^{\varepsilon}\right) \geq F_{i}(\varepsilon) .
$$

The above definition illustrates how the distribution $F_{i}$ serves as a lower bound of the actual belief player $i$ can hold about his opponents' extent of rationality. The distribution over players $-i$ 's extent of rationality induced by the belief held by $t_{i}$ must assign to each of the opponents' possible $\varepsilon$ a probability of at least $F_{i}(\varepsilon)$. For the purpose of defining types expressing $k$-fold, as well as common, belief in $F$-rationality, let $F R_{i}^{k}$ be the set of player $i$ 's types expressing $k$-fold belief in $F$-rationality, where $k \geq 1$.

A type $t_{i}$ believing in the opponents' $F$-rationality is said to express 1 -fold belief in $F$-rationality, i.e., $t_{i} \in F R_{i}^{1}$. Moreover, for any $k \geq 2$ type $t_{i}$ is said to express $k$-fold belief in $F$-rationality, written as $t_{i} \in F R_{i}^{k}$, if it only assigns positive probability to opponents' type combinations $t_{-i} \in F R_{-i}^{k-1}$ expressing $(k-1)$-fold belief in $F$-rationality. Finally, type $t_{i}$ expresses common belief in $F$-rationality, denoted by $t_{i} \in C B F R_{i}$, if it expresses $k$-fold belief in $F$-rationality for all $k \geq 1$.

Definition 2.3 Formally, $k$-fold belief in $F$-rationality and common belief in $F$-rationality can be defined recursively by the following sequence:

$$
\begin{gathered}
F R_{i}^{1}:=\left\{t_{i} \in T_{i} \mid b_{i}\left(t_{i}\right)\left(R_{-i}^{\varepsilon}\right) \geq F_{i}(\varepsilon), \forall \varepsilon \geq 0\right\} ; \\
F R_{i}^{k}:=\left\{t_{i} \in T_{i} \mid b_{i}\left(t_{i}\right)\left(C_{-i} \times F R_{-i}^{k-1}\right)=1\right\} ;
\end{gathered}
$$

and

$$
C B F R_{i}:=\bigcap_{k=1}^{\infty} F R_{i}^{k} .
$$


Note that throughout the paper, for notation simplicity and without loss of generality, we consider finite epistemic models. Still, notice that our construction can be generalized to complete epistemic models, i.e., $F R_{i}^{k}$ is well-defined for every $k>0$, if $b_{i}: T_{i} \rightarrow \triangle\left(C_{-i} \times T_{-i}\right)$ is a surjective continuous function. ${ }^{4}$ To see this, observe that $R_{-i}^{\varepsilon}$ is closed in $\left(C_{-i} \times T_{-i}\right)$, by the Portmanteau Theorem (Aliprantis and Border, 1999; Thm. 15.3). Then, it follows from Aliprantis and Border (1999; Cor. 15.6) that for an arbitrary $\varepsilon \geq 0$ it is the case that $\left\{b_{i} \in \Delta\left(C_{-i}\right) \mid b_{i}\left(R_{-i}^{\varepsilon}\right) \geq F_{i}(\varepsilon)\right\}$ is closed in $\Delta\left(C_{-i}\right)$, and therefore by continuity and completeness $F R_{i}^{1}(\varepsilon):=\left\{t_{i} \in T_{i} \mid b_{i}\left(t_{i}\right)\left(R_{-i}^{\varepsilon}\right) \geq F_{i}(\varepsilon)\right\}$ is closed in $T_{i}$. Finally, observe that $F R_{i}^{1}:=\bigcap_{\varepsilon \geq 0} F R_{i}^{1}(\varepsilon)$ is also closed, as it is the intersection of closed sets.

Following the same steps, we inductively prove that $F R_{i}^{k-1}$ is closed for every $k>1$, thus implying that $C_{-i} \times F R_{-i}^{k-1}$ is a Borel event, and therefore $b_{i}\left(t_{i}\right)\left(C_{-i} \times F R_{-i}^{k-1}\right)$ is a well-defined probability for every $t_{i} \in T_{i}$. If in addition we assume that for every $i$ the type space $T_{i}$ is compact, then $C B F R_{i}$ is non-empty. This follows from the fact that $C B F R_{i}$ contains all types satisfying common belief in rationality (for all $F_{i}$ 's) ${ }^{5}$, and the latter is non-empty whenever the $T_{i}$ 's are compact and the $b_{i}$ 's are surjective and continuous (Friedenberg and Keisler, 2011; Prop. 4.9). Of course, for an arbitrary epistemic model $C B F R_{i}$ might be empty, but the same is also true for common belief in rationality.

It is worth noting that our procedure might bear some similarity to the established $\triangle$-rationalizability of Battigalli and Siniscalchi (2003). One main aspect of similarity is that both impose exogenous restrictions on beliefs and establish common belief therein. However, one crucial difference is that the exogenous restrictions in case of $\Delta$-rationalizability are imposed on first-order beliefs, while in our model the restriction is imposed on second-order beliefs. The main restriction in our model is outlined in Definition 2.2. For a type $t_{i}$ to believe in the opponents' $F$-rationality, it has to assign at least $F_{i}(\varepsilon)$ in probability to the event $R_{-i}(\varepsilon)$ for every $\varepsilon \geq 0$. Thus our main restriction is imposed on the probability player $i$ assigns to his opponents' choice-type combinations. For any $\left(c_{-i}, t_{-i}\right) \in R_{-i}(\varepsilon)$, it must be that $c_{-i}$ is $\varepsilon$-optimal for the respective first-order beliefs held by $t_{-i}$. Hence the restriction imposed by Definition 2.2 is on the probability player $i$ assigns to sets of combinations of his opponents' choices and first-order beliefs. This core difference between our model and $\Delta$-rationalizability implies the former cannot be a special case of the latter.

\footnotetext{
${ }^{4}$ Recall that a complete epistemic model induces all belief hierarchies if the type spaces are compact and functions $b_{i}$ are continuous (Friedenberg, 2010).

${ }^{5}$ Note that the set $C B F R_{i}$ reduces in the limit to the set of types of player $i$ expressing common belief in rationality.
} 


\subsection{Iterated Elimination of $\boldsymbol{F}$-Dominated Beliefs}

Now that Common Belief in $F$-Rationality has been defined, this section develops the recursive procedure of "Iterated Elimination of $F$-Dominated Beliefs (IEFB)", to characterize those firstorder beliefs that can be held by epistemic types expressing common belief in $F$-rationality. IEFB does so by translating the basic uncertainty each player has about his opponents' rationality, bounded by $\left(F_{i}\right)_{i \in \mathcal{I}}$, into restrictions on the set of potential beliefs about opponents' choices. Thus, IE $F \mathrm{~B}$ eliminates beliefs rather than choices, with every round $k$ of the procedure resulting in a new restricted feasible belief set $B_{i}^{k}$ for each player $i$ and where $B_{i}^{0}=\Delta\left(C_{-i}\right)$.

Notably, IEFB differs from Common Belief in Rationality (CBR) in that the former eliminates beliefs which may or may not result in the elimination of some choice-combinations, while the latter proceeds directly to eliminating choice-combinations with the elimination of the belief implied. So for some choice-combination $c_{-i} \in C_{-i}$, IEFB could result in eliminating $b_{i}^{1}\left(c_{-i}\right)>a$ as a potential first-order belief, while maintaining $b_{i}^{1}\left(c_{-i}\right) \leq a$ as a valid one, where $0 \leq a \leq 1$. For Correlated Rationalizability, on the other hand, assigning positive probability to a choice-combination is either kept feasible for any $a$ or eliminated for all $a$, i.e., $a \in\{0,1\}$. IEFB can therefore be thought of as a generalization of Correlated Rationalizability, as theorem 2.2 shows. Note that Correlated Rationalizability in turn is equivalent to Iterated Elimination of Strictly Dominated Choices (IESDC).

This section is divided into two subsections. Subsection 2.3.1 formally introduces the recursive procedure and links it to IESDC. Subsection 2.3.2 then considers some practical implementation matters of the procedure.

\subsubsection{The Recursive Procedure}

Before we proceed to describing the steps of the recursive procedure, we define $\Gamma^{k}=\left(C, B^{k} ; u\right)$ to be the belief restricted game resulting from round $k$ of the procedure, i.e. it is the belief restricted game round $k+1$ of the procedure starts from where $C$ and $u$ are as defined above and $B^{k}=\left\{B_{1}^{k}, \ldots B_{n}^{k}\right\}$. Recall that $B_{i}^{k} \subseteq \Delta\left(C_{-i}\right)$ is the feasible set of player $i$ after $k$ rounds of the recursive procedure and that $B_{i}^{0}=\Delta\left(C_{-i}\right)$. Moreover, note that $\Gamma^{0}=\left(C, B^{0} ; u\right)$ is simply the original game $\Gamma$ as every $B_{i}^{0}=\Delta\left(C_{-i}\right)$ by definition.

Recall that for player $i$ to believe in his opponents' $F$-rationality, he must assign a probability of at least $F_{i}(\varepsilon)$ to the event of his opponents' having a margin of error of at most $\varepsilon$. This can 
be translated into probability distributions over opponents' choice combinations, by assigning at least $F_{i}(\varepsilon)$ to opponents' choice combinations $c_{-i}$ that are $\varepsilon$-optimal for some belief. Define the set

$$
C_{i}^{\varepsilon}\left(\Gamma^{k}\right)=\left\{c_{i} \in C_{i} \mid \exists b_{i} \in B_{i}^{k} \text { s.t. } u_{i}\left(c_{i}, b_{i}\right) \geq u_{i}\left(c_{i}^{\prime}, b_{i}\right)-\varepsilon \quad \forall c_{i}^{\prime} \in C_{i}\right\}
$$

The first step of the recursive procedure states that player $i$ must assign a probability of at least $F_{i}(\varepsilon)$ to the set of choice combinations $C_{-i}^{\varepsilon}\left(\Gamma^{0}\right)$, for every $\varepsilon \geq 0$. Beliefs satisfying the conditions $b_{i}\left(C_{-i}^{\varepsilon}\left(\Gamma^{0}\right)\right) \geq F_{i}(\varepsilon)$ for all $\varepsilon \geq 0$ constitute the feasible belief set $B_{i}^{1} \subseteq \Delta\left(C_{-i}\right)$. The set $B_{i}^{1}$ contains those first-order beliefs player $i$ can hold while believing in his opponents' $F$-rationality. Applying this for all players $i$ concludes the first round of the procedure of Iterated Elimination of $F$-Dominated Beliefs. Under up to 2-fold belief in $F$-rationality, player $i$ not only believes in his opponents' $F$-rationality, but also believes that his opponents $-i$ believe in $i$ 's $F$-rationality, making the relevant belief restricted game $\Gamma^{1}=\left(C, B^{1} ; u\right)$. Consequently, the sets $\left(C_{i}^{\varepsilon}\left(\Gamma^{0}\right)\right)_{i \in I}$ require an update, as the feasible belief sets of some players are now potentially smaller. In general, $C_{i}^{\varepsilon}\left(\Gamma^{1}\right) \subseteq C_{i}^{\varepsilon}\left(\Gamma^{0}\right)$ for every player $i \in \mathcal{I}$.

The new set $C_{-i}^{\varepsilon}\left(\Gamma^{1}\right)$ in turn implies new restrictions on the feasible belief set $B_{i}^{1}$ of player $i$ of the form $b_{i}\left(C_{-i}^{\varepsilon}\left(\Gamma^{1}\right)\right) \geq F_{i}(\varepsilon)$ for all $\varepsilon \geq 0$. These restrictions then characterize the new set $B_{i}^{2}$ of first-order beliefs player $i$ can feasibly hold under up to 2 -fold belief in $F$-rationality. The new restricted belief sets $B_{j}^{2}$ of each player $j \neq i$ can be obtained in a similar manner. The recursive procedure proceeds by iteratively eliminating beliefs and stops when $B_{i}^{k}=B_{i}^{k-1}$ for every player $i$, which may or may not happen after finitely many steps. In the latter case feasible belief sets only stabilize in the limit. The steps of round $k$ of Iterated Elimination of $F$-Dominated Beliefs are summarized below.

Procedure 2.1 Iterated Elimination of F-Dominated Beliefs:

- Initial Step: Define $\Gamma^{0}=\left(C, B^{0} ; u\right)$

- Inductive Step: Assume that $\Gamma^{k-1}=\left(C, B^{k-1} ; u\right)$ has been defined. Then $\Gamma^{k}=\left(C, B^{k} ; u\right)$ is the game where for each player $\mathrm{i}$

$$
B_{i}^{k}:=\left\{b_{i} \in \Delta\left(C_{-i}\right) \mid b_{i}\left(C_{-i}^{\varepsilon}\left(\Gamma^{k-1}\right)\right) \geq F_{i}(\varepsilon) \forall \varepsilon \geq 0\right\}
$$

Theorem 2.1 shows that the recursive procedure characterizes exactly those first-order beliefs that can be held by a type expressing common belief in $F$-rationality. 
Theorem 2.1 A belief $b_{i} \in \Delta\left(C_{-i}\right)$ can be held by a type $t_{i} \in T_{i}$ expressing common belief in $F$-rationality iff it survives all rounds of the recursive procedure of Iterated Elimination of F-Dominated Beliefs.

It is worth noting that even though the recursive procedure may not stop after finitely many rounds, the resulting sets of feasible beliefs $B_{i}^{\infty}=\bigcap_{k \geq 0} B_{i}^{k}$ surviving all rounds of the procedures, are always nonempty. This is shown in Corollary 2.1, which is based on Theorem 2.2 linking our recursive procedure to Correlated Rationalizability. Let $C_{i}^{k}$ be the choices of player $i$ surviving $k$ rounds of the procedure of iterated elimination of never-best replies characterizing Correlated Rationalizability.

Theorem 2.2 For every $k \geq 0$ and every player $i \in \mathcal{I}$, the following holds

$$
\triangle\left(C_{-i}^{k}\right) \subseteq B_{i}^{k}
$$

Note that for any finite static game, there must be some $k \geq 0$ for which $\triangle\left(C_{-i}^{k}\right)=\triangle\left(C_{-i}^{k-1}\right)$. This in turn implies the non-emptiness of the limit set of first-order beliefs surviving the procedure (Corollary 2.1).

Corollary 2.1 The limit of the feasible belief sets $B_{i}^{\infty}$ for every player $i$ is always nonempty.

Theorem 2.3 provides a fixed point characterization of the limit sets $\left(B_{i}^{\infty}\right)_{i \in I}$ of the procedure. Let $\Phi_{i} \subseteq \triangle\left(C_{-i}\right)$ be a closed and convex set of first-order beliefs of player $i$. Moreover, let $C_{i}^{\varepsilon}\left(\Phi_{i}\right)=\left\{c_{i} \in C_{i} \mid \exists \varphi_{i} \in \Phi_{i}\right.$ s.t. $\left.u_{i}\left(c_{i}, \varphi_{i}\right) \geq u_{i}\left(c_{i}^{\prime}, \varphi_{i}\right)-\varepsilon \forall c_{i}^{\prime} \in C_{i}\right\}$. Note that by definition, if $\Phi_{i}=B_{i}^{k}$ for some round $k$ of the procedure, then $C_{i}^{\varepsilon}\left(\Phi_{i}\right)=C_{i}^{\varepsilon}\left(\Gamma^{k}\right)$. We say that the collection $\left(\Phi_{i}\right)_{i \in I}$ is a best-response set if for every player $i$,

$$
\Phi_{i} \subseteq\left\{\varphi_{i} \in \Delta\left(C_{-i}\right) \mid \varphi_{i}\left(C_{-i}^{\varepsilon}\left(\Phi_{-i}\right)\right) \geq F_{i}(\varepsilon) \forall \varepsilon \geq 0\right\}
$$

Theorem 2.3 shows that the limit sets $\left(B_{i}^{\infty}\right)_{i \in \mathcal{I}}$ of first-order beliefs formed by the procedure constitute a best-response set.

Theorem 2.3 Let $\left(B_{i}^{\infty}\right)_{i \in I}$ be the limit set of first-order beliefs resulting from the procedure of Iterated Elimination of $F$-Dominated Beliefs, then $\left(B_{i}^{\infty}\right)_{i \in I}$ is a best response set.

Furthermore, Theorem 2.4 shows that the limit set $\left(B_{i}^{\infty}\right)_{i \in I}$ resulting from the procedure is also the maximal best-response set. Thus for any best response $\left(\Phi_{i}\right)_{i \in \mathcal{I}}$, with $\Phi_{i}$ as defined above for every $i$, we have $\Phi_{i} \subseteq B_{i}^{\infty}$ for all $i$.

Theorem 2.4 Let $\left(\Phi_{i}\right)_{i \in I}$ be a best response set, then $\Phi_{i} \subseteq B_{i}^{\infty}$ for every $i$. 


\subsubsection{Practical Implementation Matters}

The definition of $B_{i}^{k}$ appearing in the inductive step of the recursive procedure involves an infinite number of restrictions. However, these can be reduced to a finite number of restrictions by defining what we call "the critical $\varepsilon$ of choice $c_{i}$. Since the choice set of every player is finite, it is possible to characterize for every choice $c_{i} \in C_{i}$ within the decision problem $\Gamma^{k}$ a critical value $\varepsilon^{c_{i}}\left(\Gamma^{k}\right)$, where $\varepsilon^{c_{i}}\left(\Gamma^{k}\right)$ is the minimum value of $\varepsilon$ that makes that choice $\varepsilon$-optimal for some belief $b_{i} \in B_{i}^{k}$. Player $i$ believing in his opponents' $F$-rationality, then should assign at least probability $F_{i}\left(\varepsilon^{\prime}\right)$ to the set of choice combinations $c_{-i}$ that have a critical $\varepsilon$ of at most $\varepsilon^{\prime}$, for every $\varepsilon^{\prime} \geq 0$. These restrictions compose the new restricted belief sets $B_{i}^{k+1}$ for every player $i$.

Definition 2.4 The critical $\varepsilon$ of choice $c_{i}$ in decision problem $\Gamma^{k}$, denoted by $\varepsilon^{c_{i}}\left(\Gamma^{k}\right)$, is defined as

$$
\varepsilon^{c_{i}}\left(\Gamma^{k}\right)=\operatorname{Min}\left\{\varepsilon \mid \varepsilon \geq 0, \exists b_{i} \in B_{i}^{k} \text { with } u_{i}\left(c_{i}, b_{i}\right) \geq u_{i}\left(c_{i}^{\prime}, b_{i}\right)-\varepsilon \quad \forall c_{i}^{\prime} \in C_{i}\right\}
$$

Lemma 2.1 shows that the critical epsilon $\varepsilon^{c_{i}}\left(\Gamma^{k}\right)$ can be equivalently defined as the highest $\varepsilon$ such that choice $c_{i}$ is $\varepsilon$-strictly dominated. We say that a choice $c_{i}$ is $\varepsilon$-strictly dominated if there is an $r_{i} \in \Delta\left(C_{i}\right)$ such that $u_{i}\left(r_{i}, c_{-i}\right) \geq u_{i}\left(c_{i}, c_{-i}\right)+\varepsilon$ for all $c_{-i} \in C_{-i}$, where $r_{i}$ is a randomization assigning probability $r_{i}\left(c_{i}\right) \geq 0$ to every choice $c_{i} \in C_{i}$.

Lemma 2.1 The critical $\varepsilon^{c_{i}}\left(\Gamma^{k}\right)$ of choice $c_{i}$ in decision problem $\Gamma^{k}$ is defined as

$$
\varepsilon^{c_{i}}\left(\Gamma^{k}\right)=\operatorname{Max}\left\{\varepsilon \mid \varepsilon \geq 0, \exists r_{i} \text { with } u_{i}\left(r_{i}, b_{i}\right) \geq u_{i}\left(c_{i}, b_{i}\right)+\varepsilon \quad \forall b_{i} \in B_{i}^{k}\right\}
$$

Lemma 2.2 shows how the notion of critical $\varepsilon$ can be used to reduce the infinite set of inequalities characterizing each $B_{i}^{k}$ to a finite number of inequalities. Central to such simplification is an ascending ranking $\left\{\varepsilon_{-i}^{1}, \ldots, \varepsilon_{-i}^{M}\right\}=\left\{\varepsilon^{c_{-i}}\left(\Gamma^{k}\right) \mid c_{-i} \in C_{-i}\right\}$ of critical epsilons of the set of choice combinations $C_{-i}$, where $M=\left|C_{-i}\right|, \varepsilon_{-i}^{1}=0$ and $\varepsilon_{-i}^{1} \leq \ldots \leq \varepsilon_{-i}^{M}$.

Lemma 2.2 The infinite set of inequalities $b_{i}\left(C_{-i}^{\varepsilon}\left(\Gamma^{k}\right)\right) \geq F_{i}(\varepsilon)$ for all $\varepsilon \geq 0$ is equivalent to the finite set of inequalities of the form

$$
b_{i}\left(C_{-i}^{\varepsilon^{m}}\left(\Gamma^{k}\right)\right) \geq \operatorname{Lim}_{\varepsilon \uparrow \varepsilon_{-i}^{m+1}} F_{i}(\varepsilon) \quad \forall m \in\{1, \ldots, M-1\}
$$

Because $C_{-i}^{\varepsilon^{M}}=C_{-i}$, it follows that $b_{i}\left(C_{-i}^{\varepsilon^{M}}\right)=1$ 


\subsection{Special Cases}

In this section two special cases are considered, Common Belief in $\varepsilon$-Rationality and Common Belief in $p$-Belief in Rationality. The former is when one specific value of $\varepsilon$ receives full probability, while the latter refers to the case in which the only requirement imposed by functions $F_{i}$ is that a minimum probability of $p$ must be assigned to the event of the opponent being 0 rational.

\subsubsection{Common Belief in $\varepsilon$-Rationality}

Suppose that there exists for each player $j \neq i$ some $\varepsilon_{j}^{*}$ such that

$$
F_{i}(\varepsilon)=\left\{\begin{array}{lll}
0 & \text { if } & \varepsilon<\varepsilon_{-i}^{*} \\
1 & \text { if } & \varepsilon \geq \varepsilon_{-i}^{*}
\end{array}\right.
$$

$F$-rationality in this case reduces to Radner (1980)'s $\varepsilon$-rationality, with $\varepsilon=\varepsilon_{i}^{*}$ for every $i$. It therefore becomes possible for each player $i$ to classify his opponents' choices $C_{-i}$ into $\varepsilon_{-i}^{*}$-rational and $\varepsilon_{-i}^{*}$-irrational choices, and so allowing for both the model and the recursive procedure to be simplified. The concept of Common Belief in $F$-Rationality also reduces to Common Belief in $\varepsilon$-Rationality.

An epistemic type $t_{i} \in T_{i}$ believes in players -i's $\varepsilon$-rationality, $t_{i} \in \varepsilon R_{i}^{1}$, if it only assigns positive probability to choice-type combinations $\left(c_{-i}, t_{-i}\right)$ where $c_{-i}$ is $\varepsilon_{-i}^{*}$-optimal for $t_{-i}$. Types expressing $k \geq 2$ fold belief in $\varepsilon$-rationality, $t_{i} \in \varepsilon R_{i}^{k}$, can be defined in a manner analogous to the general case introduced in Section 2.3.

Definition 2.5 Formally, $k$-fold belief in $\varepsilon$-rationality and common belief in $\varepsilon$-rationality can be defined recursively by the following sequence:

$$
\begin{gathered}
\varepsilon R_{i}^{1}:=\left\{t_{i} \in T_{i}: b_{i}\left(t_{i}\right)\left(c_{-i}, t_{-i}\right)>0 \text { implies } c_{-i} \text { is } \varepsilon_{-i}^{*}-\text { optimal for } t_{-i}\right\} \\
\varepsilon R_{i}^{k}:=\left\{t_{i} \in T_{i}: b_{i}\left(t_{i}\right)\left(C_{-i} \times \varepsilon R_{-i}^{k-1}\right)=1\right\}
\end{gathered}
$$

and

$$
C B \varepsilon R_{i}:=\bigcap_{k=1}^{\infty} \varepsilon R_{i}^{k}
$$


The above specification of $F_{i}$ reduces the inequalities characterizing the feasible belief set in round $k$ of the recursive procedure to one single equality for each round (by Lemma 2.2), i.e.,

$$
b_{i}\left(C_{-i}^{\varepsilon_{-i}^{*}}\left(\Gamma^{k-1}\right)\right)=1
$$

The recursive procedure can therefore be simplified into one in which for every round choices $c_{i}$ of every player $i$ that possess a critical epsilon strictly greater than $\varepsilon_{i}^{*}$ are eliminated. Notably, the recursive procedure now proceeds by eliminating choices rather than beliefs. Since the set of choices for each player is finite, the procedure must stop after a finite number of rounds. Recursive Procedure 2.2 lists the steps of the now simplified procedure. Corollary 2.2 then shows the procedure works.

Procedure 2.2 Iterated Elimination of $\varepsilon$-Dominated Choices:

- Initial Step: Define $\Gamma^{0}=\left(C^{0} ; u\right)$, where $C^{0}=\left\{C_{1}, \ldots, C_{n}\right\}$ and therefore $\Gamma^{0}=\Gamma$

- Inductive Step: Assume that $\Gamma^{k-1}=\left(C_{i}^{k-1}, C_{-i}^{k-1} ; u_{i}, u_{-i}\right)$ has been defined. Then $\Gamma^{k}=$ $\left(C_{i}^{k}, C_{-i}^{k} ; u_{i}, u_{-i}\right)$ where for each player $\mathrm{i}$

$$
C_{i}^{k}=\left\{c_{i} \in C_{i} \mid \varepsilon^{c_{i}}\left(\Gamma^{k-1}\right) \leq \varepsilon_{i}^{*}\right\}
$$

Corollary 2.2 A choice $c_{i} \in C_{i}$ can rationally be made under common belief in $\varepsilon$-rationality if and only if it survives all rounds of the recursive procedure of iterated elimination of $\varepsilon$-dominated choices

\subsubsection{Common Belief in $p$-Belief in Rationality}

Another special case is that in which $F$ takes the form $F_{i}(\varepsilon)=p$ for all $\varepsilon \geq 0$ and for all players $i$. This is the case where each player assigns a probability of at least $p$ to the event of his opponents being 0 -rational, while the remaining probability $1-p$ can be assigned to any other degree(s) of rationality. Doing so, the player is said to believe in his opponents' $p$-rationality, as he assigns at least probability $p$ to his opponents choosing rationally (or being 0 -rational).

In this respect, a link should be made to an established concept in the literature, namely Common p-Belief in Rationality (Moderer and Samet 1989 and Hu 2007). Common belief in p-belief in rationality refers to the case where there is common belief in the event that every player $p$-believes in the opponents' rationality. Common $p$-Belief in Rationality, on the other hand, refers to the case where player $i$ assigns probability at least $p$ to the event of his opponents 
being 0-rational, player $i$ assigns probability at least $p$ to the event of every player $-i$ assigning probability at least $p$ to the event of $i$ being 0-rational, and so on. Common Belief in $p$-Belief in Rationality can thus be considered a strengthening, or a special case, of Common $p$-Belief in Rationality.

Types expressing $k$ fold belief in $p$-belief in rationality, written as $p R_{i}^{k}$, are defined below

Definition 2.6 Formally, $k$-fold belief in p-belief in rationality and common belief in p-belief in rationality can be defined recursively by the following sequence:

$$
\begin{gathered}
p R_{i}^{1}:=\left\{t_{i} \in T_{i}: b_{i}\left(t_{i}\right)\left(R_{-i}^{0}\right) \geq p\right\} ; \\
p R_{i}^{k}:=\left\{t_{i} \in T_{i}: b_{i}\left(t_{i}\right)\left(C_{-i} \times p R_{-i}^{k-1}\right)=1\right\} \\
C B p R_{i}:=\bigcap_{k=1}^{\infty} p R_{i}^{k} .
\end{gathered}
$$

Note that the only restriction implied by $F_{i}$ here is that $F_{i}(0)=p$. Each player $i$ 's choices can therefore be classified into two sets, $C_{i}^{0, k}$ and $C_{i}^{\neg 0, k}$. The former includes those $c_{i} \in C_{i}$ that are 0 -rational given the belief restricted game $\Gamma^{k}$ facing player $i$ in round $k$ of the recursive procedure, while the latter groups all choices that are not 0-rational given $\Gamma_{i}^{k}$. Restriction on the belief set then becomes $b_{i}\left(C_{-i}^{0, k}\right) \geq p$. Note that $C_{i}^{k}=C_{i}$ for all $k \geq 0$ and for all $i$, where $C_{i}^{k}=C_{i}^{0, k} \cup C_{i}^{\neg 0, k}$. So the recursive procedure never eliminates any choices, it only redistributes choices between the two above defined sets. Steps of the recursive procedure below are simultaneously applied to each player and repeated until $C_{i}^{0, k}=C_{i}^{0, k-1}$ and so $B_{i}^{k}=B_{i}^{k-1}$ for all $i$.

Procedure 2.3 Iterated Elimination of p-Dominated Choices:

- Initial Step: Define $\Gamma^{0}=\left(C^{0,0}, C^{\neg 0,0}, B^{0} ; u\right)$

- Inductive Step: Assume that $\Gamma^{k-1}=\left(C^{0, k-1}, C^{\neg 0, k-1}, B^{k-1} ; u\right)$ has been defined. Then $\Gamma^{k}=\left(C^{0, k}, C^{\neg 0, k}, B^{k} ; u\right)$ where for each player $i$

$$
\begin{gathered}
C_{i}^{0, k}=\left\{c_{i} \in C_{i} \mid \varepsilon^{c_{i}}\left(\Gamma^{k}\right)=0\right\} \\
C_{i}^{\neg 0, k}=\left\{c_{i} \in C_{i} \mid \varepsilon^{c_{i}}\left(\Gamma^{k}\right)>0\right\} \\
B_{i}^{k}:=\left\{b_{i} \in \Delta\left(C_{-i}\right): b_{i}\left(C_{-i}^{0, k}\right) \geq p\right\}
\end{gathered}
$$




\subsection{An Example: The Traveler's Dilemma}

This section uses the Traveler's dilemma of Basu (1994) both to illustrate the recursive procedure and to show how our model could help bring theoretical predictions closer to experimental findings. Subsection 2.5.1 uses a three price Traveler's Dilemma game to illustrate the steps of the procedure for two different functions $F_{i}$. Subsection 2.5.2 then presents some general results for the $n$-price Traveler's Dilemma under $C B F R$.

\subsubsection{Illustrating Examples of the Recursive Procedure}

To show how the recursive procedure works, we use a 3-price Traveler's Dilemma $(T D)$ game due to Basu (1994), in which both reward and penalty are set equal to 2 . We examine the resulting $B_{j}^{\infty}$ under two slightly different forms of $F_{j}$;

Case I

$$
F_{j}(\varepsilon)= \begin{cases}\varepsilon & \text { if } \varepsilon \leq 1 \\ 1 & \text { if } \varepsilon>1\end{cases}
$$

Case II

$$
F_{j}(\varepsilon)= \begin{cases}\frac{\varepsilon}{2} & \text { if } \varepsilon \leq 2 \\ 1 & \text { if } \varepsilon>2\end{cases}
$$

\section{Case I:}

Let $T D=\left\{P_{1}, P_{2} ; u_{1}, u_{2}\right\}$, where $P_{i}=\{1,2,3\}$ and

$$
u_{i}\left(p_{i}, p_{j}\right)= \begin{cases}p_{i}+2 & \text { if } p_{i}<p_{j} \\ p_{i} & \text { if } p_{i}=p_{j} \\ p_{j}-2 & \text { if } p_{i}>p_{j}\end{cases}
$$

Table 1 shows our $T D$ game in normal form

Table (1): Three Price Traveler's Dilemma (TD)

\begin{tabular}{c|ccc}
\hline & $p_{2}=1$ & $p_{2}=2$ & $p_{2}=3$ \\
\hline$p_{1}=1$ & 1,1 & $3,-1$ & $3,-1$ \\
$p_{1}=2$ & $-1,3$ & 2,2 & 4,0 \\
$p_{1}=3$ & $-1,3$ & 0,4 & 3,3 \\
\hline
\end{tabular}


Let $F_{j}$ be defined for both players as in in Case I above. Start round 1 of the recursive procedure. The relevant belief restricted game is $T D^{0}=T D$ or $T D^{0}=\left\{P_{1}, P_{2}, B_{1}^{0}, B_{2}^{0} ; u_{1}, u_{2}\right\}$ The critical values of epsilon $\varepsilon^{p_{i}}\left(T D^{0}\right)$ for each of player $i$ 's potential choices are 0,0 and $\frac{2}{3}$ for $p_{i}=1$, $p_{i}=2$ and $p_{i}=3$ respectively. Note that these critical values are the same for both players due to the symmetric nature of the game. So $C_{i}^{\varepsilon}=\{1,2\}$ for all $\varepsilon<\frac{2}{3}$ and $C_{i}^{\varepsilon}=\{1,2,3\}$ for all $\varepsilon \geq \frac{2}{3}$. From Lemma 2.2, this implies one restriction on player $j$ 's belief set

$$
b_{j}(\{1,2\}) \geq \frac{2}{3} \quad \text { or } \quad b_{j}(\{3\}) \leq \frac{1}{3}
$$

This restriction then defines $B_{j}^{1}$ to be the convex hull of four different points; namely, $B_{j}^{1}=$ $\operatorname{conv}\left(\left\{(1,0,0),(0,1,0), b_{j}^{*}, b_{j}^{* *}\right\}\right)$. Note that $B_{j}^{1}$ is also identical for both players due to the symmetry of the game. Figure 1 is a graphical representation of the feasible belief set $B_{j}^{1}$ after round 1 of the procedure.

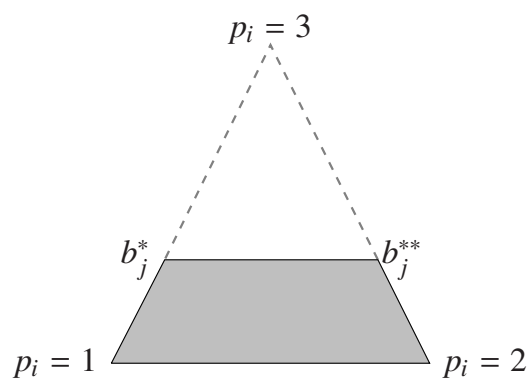

Figure 1: Feasible Belief Set $B_{j}^{1}$

Start round 2, with the belief restricted game $T D^{1}=\left\{P_{1}, P_{2}, B_{1}^{1}, B_{2}^{1} ; u_{1}, u_{2}\right\}$. Table 2 presents $T D^{1}$, where $b_{j}^{*}=\frac{1}{3}\left(p_{i}=3\right)+\frac{2}{3}\left(p_{i}=1\right)$ and $b_{j}^{* *}=\frac{1}{3}\left(p_{i}=3\right)+\frac{2}{3}\left(p_{i}=2\right)$ from Figure 1 . Note that utilities listed in Table 2 are those of player $i$ under $T D^{1}$.

Table 2: Belief Restricted Game $T D^{1}$

\begin{tabular}{c|cccc}
\hline & $p_{j}=1$ & $p_{j}=2$ & $b_{j}^{*}$ & $b_{j}^{* *}$ \\
\hline$p_{i}=1$ & 1 & 3 & $\frac{5}{3}$ & 3 \\
$p_{i}=2$ & -1 & 2 & $\frac{2}{3}$ & $\frac{8}{3}$ \\
$p_{i}=3$ & -1 & 0 & $\frac{1}{3}$ & 1 \\
\hline
\end{tabular}

The critical values of epsilon for each of both player $i$ 's choices under $T D^{1}$ become $\varepsilon_{i}^{p_{i}=1}\left(T D^{1}\right)=$ $0, \varepsilon_{i}^{p_{i}=2}\left(T D^{1}\right)=\frac{1}{3}$ and $\varepsilon_{i}^{p_{i}=3}\left(T D^{1}\right)=\frac{4}{3}$. This implies $C_{i}^{\varepsilon}=\{1\}$ for all $\varepsilon<\frac{1}{3}, C_{i}^{\varepsilon}=\{1,2\}$ for all $\frac{1}{3} \leq \varepsilon<\frac{4}{3}$ and $C_{i}^{\varepsilon}=\{1,2,3\}$ for all $\varepsilon>\frac{4}{3}$. Given $F_{j}$, restrictions on player $j$ 's feasible belief set become 


$$
\begin{array}{ccc}
b_{j}(\{1,2\}) \geq 1 & \text { or } & b_{j}(\{3\})=0 \\
b_{j}(\{1\}) \geq\left(\frac{1}{3}\right) & \text { or } & b_{j}(\{2\}) \leq\left(\frac{2}{3}\right)
\end{array}
$$

The solid line in Figure 2 represents $B_{j}^{2}=\operatorname{conv}\left(\left\{(1,0,0), b_{j}^{*}\right\}\right)$, or equivalently $B_{i}^{2}$, with $b_{j}^{*}=$ $\frac{1}{3}\left(p_{i}=1\right)+\frac{2}{3}\left(p_{i}=2\right)$.

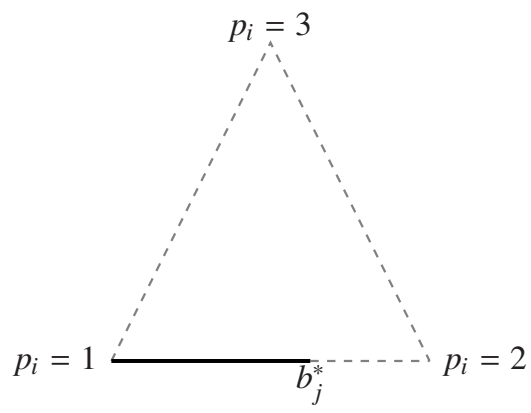

Figure 2: Feasible Belief Set $B_{j}^{2}$

Round 3 of the recursive procedure then starts with the belief restricted game $T D^{2}=\left\{P_{1}, P_{2}, B_{1}^{2}\right.$, $\left.B_{2}^{2} ; u_{1}, u_{2}\right\}$ (Table 3 ). The critical values of epsilon become the following: $\varepsilon_{i}^{p_{i}=1}\left(T D^{2}\right)=0$ and $\varepsilon_{i}^{p_{i}=2}\left(T D^{2}\right)=\frac{4}{3}$, implying that $p_{i}=2$ receives no positive probability similarly to $p_{i}=3$ in round 2. The feasible belief set $B_{j}^{3}$ thus collapses to one point assigning probability 1 to the opponent $i$ playing price 1 . The procedure stops after 3 rounds and feasible belief sets stabilize to $B_{j}^{3}=B_{j}^{\infty}=\left\{b_{j}\left(p_{i}=1\right)=1\right\}$ for both players.

Table 3: Belief Restricted Game $T D^{2}$

\begin{tabular}{c|cc}
\hline & $p_{j}=1$ & $b_{j}^{*}$ \\
\hline$p_{i}=1$ & 1 & $7 / 3$ \\
$p_{i}=2$ & -1 & 1 \\
\hline
\end{tabular}

\section{Case II:}

Let the game $T D$ be as previously defined, and consider the slightly modified form of the $F_{j}$ function corresponding to Case II. Rounds of the recursive procedure then proceed as follows: Round 1 starts with the same $T D^{0}$ as above with the critical epsilons being $\varepsilon_{i}^{p_{i}=1}\left(T D^{0}\right)=$ $\varepsilon_{i}^{p_{i}=2}\left(T D^{0}\right)=0$ and $\varepsilon_{i}^{p_{i}=3}\left(T D^{0}\right)=\frac{2}{3}$ for both players. The current function $F_{j}(\varepsilon)$, however, implies different restrictions on the feasible belief set $B_{j}^{1}$, which take the form 


$$
b_{j}(\{1,2\}) \geq\left(\frac{1}{3}\right) \quad \text { or } \quad b_{j}(\{3\}) \leq\left(\frac{2}{3}\right)
$$

Figure 3 below represents the feasible belief set $B_{j}^{1}$ after round 1 of the procedure.

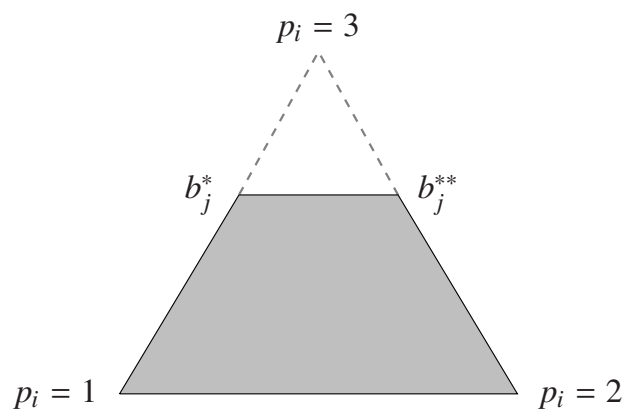

Figure 3: Feasible Belief Set $B_{j}^{1}$

Round 2 starts with the belief restricted game $T D^{1}=\left\{P_{1}, P_{2}, B_{1}^{1}, B_{2}^{1} ; u_{1}, u_{2}\right\}$. Table 4 presents $T D^{1}$, where $b_{j}^{*}=\left(\frac{2}{3}\right)\left(p_{i}=3\right)+\left(\frac{1}{3}\right)\left(p_{i}=1\right)$ and $b_{j}^{* *}=\left(\frac{2}{3}\right)\left(p_{i}=3\right)+\left(\frac{1}{3}\right)\left(p_{i}=2\right)$ from Figure 3 .

Table 4: Belief Restricted Game $T D^{1}$

\begin{tabular}{c|cccc}
\hline & $p_{j}=1$ & $p_{j}=2$ & $b_{j}^{*}$ & $b_{j}^{* *}$ \\
\hline$p_{i}=1$ & 1 & 3 & $\frac{7}{3}$ & 3 \\
$p_{i}=2$ & -1 & 2 & $\frac{7}{3}$ & $\frac{10}{3}$ \\
$p_{i}=3$ & -1 & 0 & $\frac{5}{3}$ & 2 \\
\hline
\end{tabular}

From Table 4, it is evident that the critical epsilons of all three choices remain unchanged. Consequently, the restrictions on player $j$ 's feasible belief set, and so the belief set $B_{j}^{2}$ itself remain unchanged as well. By symmetry of the game, the same holds for player $i$. The recursive procedure stops after two rounds as $B_{j}^{2}=B_{j}^{1}$ for both players. The set of first-order beliefs that can be held by a type expressing common belief in $F$-rationality in the 3-price $T D$ game at hand, thus contains an infinite set of beliefs assigning positive probabilities to both the second and third price.

\subsubsection{CBFR in the Traveler's Dilemma}

As pointed out earlier, the model we introduce along with the characterizing procedure is purely concerned with players' beliefs and does not by itself say anything about the actual choices players 
would make in a given game, as the actual $\varepsilon$ of each player is his/ her private information. Take for example the case II in the previous section. Although the feasible belief set of each player $i$ contains beliefs assigning probability up to $\frac{2}{3}$ to the opponent's highest price, player $i$ can still react to such a belief by playing a 0-optimal choice. The main message of this section is to show that our model can account for some of the experimental observations in the Traveler's Dilemma even with very little deviation from rationality and common belief in rationality. This is achieved by allowing each player to account for the possibility that his opponent may make a mistake with some probability.

Consider an $n$-price Traveler's Dilemma where each player $i$ chooses $p_{i} \in\{1, \ldots, n\}$. The critical epsilon $\varepsilon^{n}\left(T D^{0}\right)$ of the highest price $n$ is at most $\frac{2}{n}$ while that of any $p_{i} \neq n$ is 0 . To show that $\varepsilon^{n}\left(T D^{0}\right)=\frac{2}{n}$, note that for an $n$-price game with reward and punishment set equal to 2 , the following holds

$$
u_{i}\left(p_{i}, 1\right)= \begin{cases}1 & \text { for } p_{i}=1 \\ -1 & \text { otherwise }\end{cases}
$$

and

$$
u_{i}\left(p_{i}, n\right)= \begin{cases}p_{i}+2 & \text { for } p_{i}<n \\ n & \text { for } p_{i}=n\end{cases}
$$

Consider the belief $b_{i}$ of player $i$ assigning probability $\frac{2}{n}$ to the opponent's choice $p_{j}=n$ and $1-\frac{2}{n}$ to $p_{j}=1$. The expected utilities of player $i$ 's choices given such belief are as follows: $u_{i}\left(1, b_{i}\right)=1+\frac{4}{n}, u_{i}\left(n, b_{i}\right)=1+\frac{2}{n}$ and $u_{i}\left(p_{i}, b_{i}\right)=\frac{2}{n}\left(p_{i}+3\right)-1$ for prices $1<p_{i}<n$. Note that the highest $u_{i}\left(p_{i}, b_{i}\right)$ for $1<p_{i}<n$ is that of price $n-1$. It is easy to see that $u_{i}\left(n, b_{i}\right)$ is exactly $\frac{2}{n}$ lower than the expected utility of the best response choice. Thus there exists a belief $b_{i}$ for which $n$ is $\frac{2}{n}$-optimal, thereby proving that $\frac{2}{n}$ is at least and upper bound on $\varepsilon^{n}\left(T D^{0}\right)$. Note that the upper bound on the critical epsilon is a decreasing function in the number of prices. Thus the higher the number of prices the smaller the loss in expected utility a player has to (be believed to) tolerate to choose a higher price. Now assume $F_{i}$ is defined for players $i=\{1,2\}$ as follows:

$$
F_{i}(\varepsilon)= \begin{cases}\frac{n-2}{2} \varepsilon & \text { if } \varepsilon \leq \frac{2}{n-2} \\ 1 & \text { if } \varepsilon>\frac{2}{n-2}\end{cases}
$$

Since $\frac{2}{n}$ constitutes an upper limit on the critical epsilon $\varepsilon^{n}\left(T D^{0}\right)$ of price $p_{j}=n$, then the restriction imposed on player $i$ 's feasible belief set in round 1 of the procedure based on the actual $\varepsilon^{n}\left(T D^{0}\right)$ must satisfy $b_{i}(n) \leq \frac{2}{n}$. Given this restriction, one of the extreme points in the 
belief restricted game $T D^{1}$ defined in round 1 for player $i$ becomes $b_{i}^{1 n}=\frac{2}{n}(n)+\left(1-\frac{2}{n}\right)(1)$. In $T D^{1}$, choice $n$ of player $i$ is $\frac{2}{n}$-optimal for $b_{i}^{1 n}$ as shown above. The critical $\varepsilon^{n}\left(T D^{1}\right) \leq \frac{2}{n}$. This implies no further restrictions will be imposed on player $i$ 's belief set in round 2 of the procedure.

Assume player $i=\{1,2\}$ is 0 -rational and holds the belief $b_{i}^{1 n} \in B_{i}^{\infty}$ about his opponent's choices. Player $i$ 's best response is then to choose $n-1$ under CBFR. Thus even if each player is still 0-rational, assigning positive probability to the opponent making mistakes of no more than $\frac{2}{n-2}$ in expected utility in an $n$-price game, might lead the player to choose for a much higher price than predicted by CBR. Note that the maximum extent of irrationality $\frac{2}{n-2}$ that must be deemed possible is also a decreasing function in $n$.

\subsection{Discussion and Conclusion}

By allowing players to be boundedly rational and introducing uncertainty about opponents' extent of rationality/ irrationality, this paper develops the concept of Common Belief in $F$-Rationality. A recursive procedure is also designed to characterize exactly those first-order beliefs that can be held by an epistemic type expressing common belief in $F$-rationality. The recursive procedure of Iterated Elimination of $F$-Dominated Beliefs restricts each player's belief set by eliminating those beliefs that are inconsistent with the event of believing in the opponents' $F$-rationality. Finally, we also consider two special cases of the function $F$, which correspond to Common Belief in $\varepsilon$-Rationality and Common Belief in $p$-Belief in Rationality. Both the model and the recursive procedure are illustrated for these special cases. Two issues remain to be discussed.

First, our model attempted to capture the possibility that a player would make mistakes or believe his opponents' to do so, taking into account that the extent of these mistakes is at best private information. The uncertainty about opponents' potential mistakes is regulated by means of the $F$-functions assigning to each potential margin of error a maximum level of probability. The game considered was one of complete information. We believe restrictions on beliefs similar to the ones characterized by our model could be obtained had we considered games of incomplete information in which the utility functions of the players are not transparent. Instead of a margin of error, $\varepsilon$ would be redefined as the maximum distance between different possible utility functions of the opponent and his true utility function in a manner similar to that of Perea and Roy (2014). The minimum bound on probability $F_{i}(\varepsilon)$ would then also be reinterpreted accordingly. The set up of the model and procedure of the incomplete information case is expected to bear many similarities to the model presented in this paper. However, the extent of such similarities is beyond the scope of this paper and is a question for further research. 
Second, it is important to stress that our model remains silent about the way players actually act upon their beliefs. Players in our model are not assumed to be $\varepsilon$-best responders. In fact, players may actually make perfectly rational choices in response to the beliefs they hold under common belief in $F$-rationality. Thus the model only assumes that players believe that their opponents might make errors causing them to deviate from their optimal choices. This has been made evident in the Traveler's Dilemma section where players assigning positive probability to the event of their opponent making mistakes up to a certain extent has led even 0-rational players to plausibly choosing prices up to the second highest. This example serves as an indication that some of the observed experimental results, at least in the Traveler's Dilemma game, may be explained by slight deviations from standard rationality. 


\subsection{Appendix}

Proof of Theorem 2.1: The following two statements are to be proven:

(A) Any $b_{i}\left(t_{i}\right)$ with $t_{i} \in C B F R_{i}$ has $b_{i}^{1}\left(t_{i}\right) \in B_{i}^{\infty}$

(B) Any $b_{i}^{1}\left(t_{i}\right) \in B_{i}^{\infty}$ can be held by a type $t_{i} \in C B F R_{i}$

Part A: We prove by induction on $k$ that $b_{i}^{1}\left(t_{i}\right) \in B_{i}^{k}$ for all $k$, whenever $t_{i} \in C B F R_{i}$. Let $k=0$. Take some $t_{i} \in C B F R_{i}$, then $b_{i}^{1}\left(t_{i}\right) \in B_{i}^{0}$, where $B_{i}^{0}=\triangle\left(C_{-i}\right)$. Let $k \geq 1$. Assume for every $t_{i} \in C B F R_{i}, b_{i}^{1}\left(t_{i}\right) \in B_{i}^{k-1}$ for both players $i$, where $B_{i}^{k-1}=\left\{b_{i} \in \triangle\left(C_{-i}\right) \mid b_{i}\left(C_{-i}^{\varepsilon}\left(\Gamma^{k-2}\right)\right) \geq\right.$ $\varepsilon$ for all $\varepsilon \geq 0\}$.

Take some $t_{i} \in C B F R_{i}$, then

(i) $b_{i}\left(t_{i}\right)\left(R_{-i}^{\varepsilon}\right) \geq F_{i}(\varepsilon)$ as $t_{i} \in F R_{i}^{1}$ for all $\varepsilon \geq 0$

(ii) $b_{i}\left(t_{i}\right)\left(c_{-i}, t_{-i}\right)>0$ implies $t_{-i} \in C B F R_{-i}$

From the induction assumption above, it follows that $b_{-i}^{1}\left(t_{-i}\right) \in B_{-i}^{k-1}$ whenever $t_{-i} \in C B F R_{-i}$. Let $R_{-i}^{\varepsilon, k-1}:=\left\{\left(c_{-i}, t_{-i}\right) \in R_{-i}^{\varepsilon}\left(\Gamma^{k-1}\right) \mid b_{-i}^{1}\left(t_{-i}\right) \in B_{-i}^{k-1}\right\}$, where $R_{-i}^{\varepsilon}:=\left\{\left(c_{-i}, t_{-i}\right) \mid c_{-i}\right.$ is $\varepsilon$-optimal for $\left.b_{-i}^{1}\left(t_{-i}\right)\right\}$.

Then (i) and (ii) imply, $b_{i}\left(t_{i}\right)\left(R_{-i}^{\varepsilon, k-1}\right) \geq F_{i}(\varepsilon)$ for all $\varepsilon \geq 0$. So $b_{i}\left(t_{i}\right)\left(\operatorname{Proj}_{C_{-i}} R_{-i}^{\varepsilon, k-1} \times\right.$ $\left.T_{-i}\right) \geq F_{i}(\varepsilon)$ for all $\varepsilon \geq 0$, which means $b_{i}^{1}\left(t_{i}\right)\left(\operatorname{Proj}_{C_{-i}} R_{-i}^{\varepsilon, k-1}\right) \geq F_{i}(\varepsilon)$ for all $\varepsilon \geq 0$. Since $\operatorname{Proj}_{C_{-i}} R_{-i}^{\varepsilon, k-1}=C_{-i}^{\varepsilon}\left(\Gamma^{k-1}\right)$, it follows that $b_{i}^{1}\left(t_{i}\right)\left(C_{-i}^{\varepsilon}\left(\Gamma^{k-1}\right)\right) \geq F_{i}(\varepsilon)$ for all $\varepsilon \geq 0$, and therefore $b_{i}^{1}\left(t_{i}\right) \in B_{i}^{k}$.

Part B: This part is proven in two steps, both of which require a construction of an epistemic model. Consider a finite epistemic model $M$ with the set of types $T_{i}$ for every player $i$. The set $T_{i}$ is constructed as follows:

For every $c_{i} \in C_{i}$ choose some $b_{i}^{c_{i}} \in B_{i}^{\infty}$ such that $c_{i}$ is exactly $\varepsilon^{c_{i}}\left(\Gamma^{\infty}\right)$-optimal for $b_{i}^{c_{i}}$. Define the finite set of types $T_{i}^{C_{i}}=\left\{t_{i}^{c_{i}} \mid c_{i} \in C_{i}\right\}$ where every $t_{i}^{c_{i}} \in T_{i}^{C_{i}}$ is such that

$$
b_{i}\left(t_{i}^{c_{i}}\right)\left(c_{-i}, t_{-i}\right)= \begin{cases}b_{i}^{c_{i}}\left(c_{-i}\right) & \text { if } t_{-i}=t_{-i}^{c_{-i}} \\ 0 & \text { Otherwise }\end{cases}
$$


Let $b_{i}^{*}$ be some arbitrary belief in $B_{i}^{\infty}$. Then define the finite set of types $T_{i}=T_{i}^{C_{i}} \cup\left\{t_{i}^{*}\right\}$ where $t_{i}^{*}$ is such that

$$
b_{i}\left(t_{i}^{*}\right)\left(c_{-i}, t_{-i}\right)= \begin{cases}b_{i}^{*}\left(c_{-i}\right) & \text { if } t_{-i}=t_{-i}^{c_{-i}} \\ 0 & \text { Otherwise }\end{cases}
$$

Step 1: Take some $t_{i}^{c_{i}} \in T_{i}^{C_{i}}$. Since $b_{i}^{1}\left(t_{i}^{c_{i}}\right)=b_{i}^{c_{i}}$ and $b_{i}^{c_{i}} \in B_{i}^{\infty}$, it follows that

$$
b_{i}^{1}\left(t_{i}^{c_{i}}\right)\left(C_{-i}^{\varepsilon}\left(\Gamma^{\infty}\right)\right)=b_{i}^{c_{i}}\left(C_{-i}^{\varepsilon}\left(\Gamma^{\infty}\right)\right) \geq F_{i}(\varepsilon) \quad \text { for all } \quad \varepsilon \geq 0
$$

Take some $c_{-i} \in C_{-i}^{\varepsilon}\left(\Gamma^{\infty}\right)$, then by definition of $C_{-i}^{\varepsilon}\left(\Gamma^{k}\right)$ it must be that $\varepsilon^{c_{-i}}\left(\Gamma^{\infty}\right) \leq \varepsilon$. Since $c_{-i}$ is $\varepsilon^{c_{-i}}\left(\Gamma^{\infty}\right)$-optimal for $t_{-i}^{c_{-i}}$, it is also $\varepsilon$-optimal for $t_{-i}^{c_{-i}}$ for any $\varepsilon \geq \varepsilon^{c_{-i}}\left(\Gamma^{\infty}\right)$. So $\left(c_{-i}, t_{-i}^{c_{-i}}\right) \in R_{-i}^{\varepsilon}$. Hence

$$
b_{i}\left(t_{i}^{c_{i}}\right)\left(R_{-i}^{\varepsilon}\right) \geq b_{i}^{1}\left(t_{i}^{c_{i}}\right)\left(C_{-i}^{\varepsilon}\left(\Gamma^{\infty}\right)\right) \geq F_{i}(\varepsilon) \quad \text { for all } \quad \varepsilon \geq 0
$$

So $t_{i}^{c_{i}} \in F R_{i}^{1}$. Condition (1) implies $t_{i}^{c_{i}}$ only assigns positive probability to $t_{-i}^{c_{-i}}$, which in turn only assign positive probability to $t_{i}^{c_{i}}$. Since $t_{i}^{c_{i}} \in F R_{i}^{1}$ for both players $i$, it follows that $t_{i}^{c_{i}} \in C B F R_{i}$.

Step 2: Consider $t_{i}^{*} \in T_{i}$. Since $t_{i}^{c_{i}} \in C B F R_{i}$ for both players $i$ (Step 1), it follows from condition (2) that $t_{i}^{*}$ only assigns positive probability to $\left(c_{-i}, t_{-i}\right)$ where $t_{-i} \in C B F R_{-i}$. It therefore suffices to show that $t_{i}^{*} \in F R_{i}^{1}$.

Since $b_{i}^{*} \in B_{i}^{\infty}$,

$$
b_{i}^{1}\left(t_{i}^{*}\right)\left(C_{-i}^{\varepsilon}\left(\Gamma^{\infty}\right)\right) \geq F_{i}(\varepsilon) \quad \text { for all } \quad \varepsilon \geq 0
$$

Since $t_{i}^{*}$ satisfies by definition condition (2) and so only assigns positive probability to $\left(c_{-i}, t_{-i}\right)$ where $t_{-i}=t_{-i}^{c_{-i}}$, the same steps underlined in Step 1 above can be followed from this point onward leading to the conclusion that $t_{i}^{*} \in F R_{i}^{1}$.

Proof of Theorem 2.2: This requires proving that for every $k \geq 0$ and every player $i \in \mathcal{I}$, the following holds $B_{i}^{C_{-i}^{k}} \subseteq B_{i}^{k}$, where $B_{i}^{C_{-i}^{k}}=\Delta\left(C_{-i}^{k}\right)$ and where $C_{i}^{k}$ is the set of choices of player $i$ surviving $k$ rounds of the procedure of iterated elimination of never-best replies characterizing Correlated Rationalizability. This will be proven by induction.

Induction Start: Take some $b_{i} \in \triangle\left(C_{-i}^{0}\right)$ then it is also the case that $b_{i} \in \Delta\left(C_{-i}\right)$. By definition of $B_{i}^{0}$, it follows that $b_{i} \in B_{i}^{0}$. 
Induction Assumption: For any $b_{i} \in \Delta\left(C_{-i}^{k-1}\right)$ it is also the case that $b_{i} \in B_{i}^{k-1}$.

Induction Step: Take some $b_{i} \in \Delta\left(C_{-i}^{k}\right)$. Since $C_{-i}^{k} \subseteq C_{-i}^{k-1}$ by definition of $C_{-i}^{k}$. Then it follows that $b_{i} \in B_{i}^{k-1}$. Recall that $C_{i}^{\varepsilon}\left(\Gamma^{k-1}\right)=\left\{c_{i} \in C_{i} \mid c_{i}\right.$ is $\varepsilon-$ optimal for some $\left.b_{i} \in B_{i}^{k-1}\right\}$. Moreover, any $c_{i} \in C_{i}^{k}$ is 0 -optimal for some $b_{i} \in \Delta\left(C_{-i}^{k-1}\right)$. From the induction assumption, the latter can be rewritten as: any $c_{i} \in C_{i}^{k}$ is 0 -optimal for some $b_{i} \in B_{i}^{k-1}$. So $C_{-i}^{k} \in C_{-i}^{0}\left(\Gamma^{k-1}\right)$. Since any $b_{i} \in \Delta\left(C_{-i}^{k}\right)$ is also such that $b_{i}\left(C_{-i}^{k}\right)=1$, and since $C_{-i}^{k} \in C_{-i}^{0}\left(\Gamma^{k-1}\right)$, it follows that $b_{i}\left(C_{-i}^{0}\left(\Gamma^{k-1}\right)\right)=1$. So $b_{i} \in B_{i}^{k}$.

Proof of Theorem 2.3: Let the collection $\left(B_{i}^{\infty}\right)_{i \in \mathcal{I}}$ be the limit sets of first-order beliefs surviving the procedure of IEFB. Let $\left(\Phi_{i}\right)_{i \in I}$ be a best-response set where for every player $i$,

$$
\Phi_{i} \subseteq\left\{\varphi_{i} \in \Delta\left(C_{-i}\right) \mid \varphi_{i}\left(C_{-i}^{\varepsilon}\left(\Phi_{-i}\right)\right) \geq F_{i}(\varepsilon) \forall \varepsilon \geq 0\right\}
$$

and

$$
C_{i}^{\varepsilon}\left(\Phi_{i}\right)=\left\{c_{i} \in C_{i} \mid \exists \varphi_{i} \in \Phi_{i} \text { s.t. } u_{i}\left(c_{i}, \varphi_{i}\right) \geq u_{i}\left(c_{i}^{\prime}, \varphi_{i}\right)-\varepsilon \forall c_{i}^{\prime} \in C_{i}\right\}
$$

Take some $b_{i} \in B_{i}^{\infty}$. Then by definition of $B_{i}^{k}, b_{i}\left(C_{-i}^{\varepsilon}\left(\Gamma^{\infty}\right)\right) \geq F_{i}(\varepsilon)$ for all $\varepsilon \geq 0$. By definition, $C_{j}^{\varepsilon}\left(\Gamma^{\infty}\right)=C_{j}^{\varepsilon}\left(B_{j}^{\infty}\right)$ for any $j$. Since $b_{i} \in B_{i}^{\infty}$, it follows that

$$
b_{i}\left(C_{-i}^{\varepsilon}\left(B_{-i}^{\infty}\right)\right) \geq F_{i}(\varepsilon) \forall \varepsilon \geq 0
$$

Note that condition $(*)$ is identical to that defining best-response sets $\left(\Phi_{i}\right)_{i \in \mathcal{I}}$. Since condition (*) holds for any $b_{i} \in B_{i}^{\infty}$ and for every player $i$, it follows that $\left(B_{i}^{\infty}\right)_{i \in \mathcal{I}}$ is a best-response set.

\section{Proof of Theorem 2.4:}

Consider some best response set $\left(\Phi_{i}\right)_{i \in \mathcal{I}}$ where for every player $i$,

$$
\Phi_{i} \subseteq\left\{\varphi_{i} \in \Delta\left(C_{-i}\right) \mid \varphi_{i}\left(C_{-i}^{\varepsilon}\left(\Phi_{-i}\right)\right) \geq F_{i}(\varepsilon) \forall \varepsilon \geq 0\right\}
$$

and

$$
C_{i}^{\varepsilon}\left(\Phi_{i}\right)=\left\{c_{i} \in C_{i} \mid \exists \varphi_{i} \in \Phi_{i} \text { s.t. } u_{i}\left(c_{i}, \varphi_{i}\right) \geq u_{i}\left(c_{i}^{\prime}, \varphi_{i}\right)-\varepsilon \forall c_{i}^{\prime} \in C_{i}\right\}
$$

For every round $k \geq 1$ of the procedure, we prove by induction on $k$ that for any best response set $\left(\Phi_{i}\right)_{i \in \mathcal{I}}, \Phi_{i} \subseteq B_{i}^{k}$ holds for every $i$. First take $k=1$ and show that $\Phi_{i} \subseteq B_{i}^{1}$ for every $i$. Then by induction on $k$ we show that $\Phi_{i} \subseteq B_{i}^{k}$ for any $k$ and every $i$. Recall that

$$
B_{i}^{1}=\left\{b_{i} \in \Delta\left(C_{-i}\right) \mid b_{i}\left(C_{-i}^{\varepsilon}\left(\Gamma^{0}\right)\right) \geq F_{i}(\varepsilon) \quad \forall \varepsilon \geq 0\right\} .
$$


By definitions of $\Gamma^{0}$ and $\Phi_{i}$, it follows for every player $j$ that $C_{j}^{\varepsilon}\left(\Phi_{j}\right) \subseteq C_{j}^{\varepsilon}\left(B_{j}^{0}\right)=C_{j}^{\varepsilon}\left(\Gamma^{0}\right)$ for every $\varepsilon \geq 0$ and for any $\Phi_{j}$. Take some $\varphi_{i} \in \Phi_{i}$, then by definition

$$
\varphi_{i}\left(C_{-i}^{\varepsilon}\left(\Phi_{-i}\right)\right) \geq F_{i}(\varepsilon) \forall \varepsilon \geq 0
$$

Hence,

$$
\varphi_{i}\left(C_{-i}^{\varepsilon}\left(B_{-i}^{0}\right)\right) \geq \varphi_{i}\left(C_{-i}^{\varepsilon}\left(\Phi_{-i}\right)\right) \geq F_{i}(\varepsilon) \forall \varepsilon \geq 0
$$

Thus, $\Phi_{i} \subseteq B_{i}^{1}$. Assume that $\Phi_{i} \subseteq B_{i}^{k-1}$ for all $i$. Let $k \geq 2$ and show that $\Phi_{i} \subseteq B_{i}^{k}$ for all $i$ and for any $k$. Proving that requires showing that every $\varphi_{i} \in \Phi_{i}$ is such that

$$
\varphi_{i}\left(C_{-i}^{\varepsilon}\left(\Gamma^{k-1}\right)\right) \geq F_{i}(\varepsilon) \forall \varepsilon \geq 0
$$

Since $\Phi_{j} \subseteq B_{j}^{k-1}$ for all $j$ by the induction assumption, then for every player $j$

$$
C_{j}^{\varepsilon}\left(\Phi_{j}\right) \subseteq C_{j}^{\varepsilon}\left(B_{j}^{k-1}\right)=C_{j}^{\varepsilon}\left(\Gamma^{k-1}\right) \quad \forall \varepsilon \geq 0
$$

Hence,

$$
\varphi_{i}\left(C_{-i}^{\varepsilon}\left(B_{-i}^{k-1}\right)\right) \geq \varphi_{i}\left(C_{-i}^{\varepsilon}\left(\Phi_{-i}\right)\right) \geq F_{i}(\varepsilon) \quad \forall \varepsilon \geq 0
$$

Thus, $\Phi_{i} \subseteq B_{i}^{k}$ for any $k \geq 2$ for every $i$. Since this holds for every $k$, then by definition of $B_{i}^{\infty}$, it follows that $\Phi_{i} \subseteq B_{i}^{\infty}$ for every player $i$.

Proof of Lemma 2.1: Let $C_{i}^{\varepsilon}\left(\Gamma^{k}\right)=\left\{c_{i} \in C_{i}: \varepsilon^{c_{i}}\left(\Gamma^{k}\right) \leq \varepsilon\right\}$, and let $B_{i}^{k}:=\left\{b_{i} \in \Delta\left(C_{-i}\right) \mid\right.$ $\left.b_{i}\left(C_{-i}^{\varepsilon}\left(\Gamma^{k-1}\right)\right) \geq F_{i}(\varepsilon) \forall \varepsilon \geq 0\right\}$. Since $B_{i}^{k}$ can be defined by finitely many linear inequalities (Lemma 2.2), it is possible to define the set $E B_{i}^{k}:=\left\{b_{i} \in B_{i}^{k} \mid b_{i}\right.$ is an extreme point of $\left.B_{i}^{k}\right\}$. Note that since $B_{i}^{0}=\triangle\left(C_{-i}\right), E B_{i}^{0}:=\left\{b_{i} \in \Delta\left(C_{-i}\right) \mid b_{i}\left(c_{-i}\right)=1\right.$ for some $\left.c_{-i} \in C_{-i}\right\}$. Lemma 2.1then requires proving:

(A) For every $c_{i}^{*} \notin C_{i}^{\varepsilon}\left(\Gamma^{k}\right)$ for some $\varepsilon \geq 0$, there exists $r_{i} \in \Delta\left(C_{i} \backslash\left\{c_{i}^{*}\right\}\right)$ such that $u_{i}\left(r_{i}, b_{i}\right)>$ $u_{i}\left(c_{i}^{*}, b_{i}\right)+\varepsilon$ for all $b_{i} \in B_{i}^{k}$.

(B) For every $c_{i}^{*}$ for which there exists $r_{i} \in \Delta\left(C_{i} \backslash\left\{c_{i}^{*}\right\}\right)$ such that $u_{i}\left(r_{i}, b_{i}\right)>u_{i}\left(c_{i}^{*}, b_{i}\right)+\varepsilon$ for all $b_{i} \in B_{i}^{k}$, it is also the case that $c_{i}^{*} \notin C_{i}^{\varepsilon}\left(\Gamma^{k}\right)$.

(C) For $c_{i}^{*} \in C_{i}$ and for some given $k$, the critical epsilon $\varepsilon^{c_{i}^{*}}\left(\Gamma^{k}\right)$ is defined by

$$
\varepsilon^{c_{i}^{*}}\left(\Gamma^{k}\right)=\operatorname{Max}\left\{\varepsilon \mid \varepsilon \geq 0, \exists r_{i} \text { with } u_{i}\left(r_{i}, b_{i}\right) \geq u_{i}\left(c_{i}^{*}, b_{i}\right)+\varepsilon \quad \forall b_{i} \in B_{i}^{k}\right\}
$$


Part A: Let $c_{i}^{*} \notin C_{i}^{\varepsilon}$ for some $\varepsilon \geq 0$, then for every $b_{i} \in B_{i}^{k}$, there is some $c_{i}$ such that

$$
u_{i}\left(c_{i}, b_{i}\right)>u_{i}\left(c_{i}^{*}, b_{i}\right)+\varepsilon
$$

Let $\Gamma_{\varepsilon}^{k}$ be a modified game of $\Gamma^{k}$ with transformed utilities as follows:

For $c_{i}^{*} \in C_{i} \quad u_{i}^{\varepsilon}\left(c_{i}^{*}, b_{i}\right)=u\left(c_{i}^{*}, b_{i}\right)+\varepsilon \quad \forall b_{i} \in B_{i}^{k}$

For every $c_{i} \in C_{i} \backslash\left\{c_{i}^{*}\right\} \quad u_{i}^{\varepsilon}\left(c_{i}, b_{i}\right)=u_{i}\left(c_{i}, b_{i}\right) \quad \forall b_{i} \in B_{i}^{k}$

So for every $b_{i} \in B_{i}^{k}$, there exists some $c_{i}$ such that

$$
u_{i}^{\varepsilon}\left(c_{i}, b_{i}\right)>u_{i}^{\varepsilon}\left(c_{i}^{*}, b_{i}\right)
$$

Lemma 3 in Pearce (1984) then implies, there exists $r_{i} \in \Delta\left(C_{i} \backslash\left\{c_{i}^{*}\right\}\right)$ such that

$$
u_{i}^{\varepsilon}\left(r_{i}, b_{i}\right)>u_{i}^{\varepsilon}\left(c_{i}^{*}, b_{i}\right) \quad \forall b_{i} \in E B_{i}^{k}
$$

Or equivalently,

$$
u_{i}\left(r_{i}, b_{i}\right)>u_{i}\left(c_{i}^{*}, b_{i}\right)+\varepsilon \quad \forall b_{i} \in E B_{i}^{k}
$$

By the convexity of $B_{i}^{k}$, it follows there is some $r_{i} \in \Delta\left(C_{i} \backslash\left\{c_{i}^{*}\right\}\right)$ such that

$$
u_{i}\left(r_{i}, b_{i}\right)>u_{i}\left(c_{i}^{*}, b_{i}\right)+\varepsilon \quad \forall b_{i} \in B_{i}^{k}
$$

Part B: Consider some $c_{i}^{*}$ for which there exists $r_{i} \in \Delta\left(C_{i}\right)$ such that

$$
u_{i}\left(r_{i}, b_{i}\right)>u_{i}\left(c_{i}^{*}, b_{i}\right)+\varepsilon \quad \text { for all } \quad b_{i} \in B_{i}^{k}
$$

Since $E B_{i}^{k} \subset B_{i}^{k}, c_{i}^{*}$ is also such that there is some $r_{i} \in \triangle\left(C_{i}\right)$ with

$$
u_{i}\left(r_{i}, b_{i}\right)>u_{i}\left(c_{i}^{*}, b_{i}\right)+\varepsilon \quad \text { for all } \quad b_{i} \in E B_{i}^{k}
$$


In $\Gamma_{\varepsilon}^{k}$ this becomes,

$$
u_{i}^{\varepsilon}\left(r_{i}, b_{i}\right)>u_{i}^{\varepsilon}\left(c_{i}^{*}, b_{i}\right) \quad \text { for all } \quad b_{i} \in E B_{i}^{k}
$$

Lemma 3 in Pearce (1984) then implies for every $b_{i} \in B_{i}^{k}$ there exists $c_{i} \in C_{i} \backslash\left\{c_{i}^{*}\right\}$ such that

$$
u_{i}^{\varepsilon}\left(c_{i}, b_{i}\right)>u_{i}^{\varepsilon}\left(c_{i}^{*}, b_{i}\right)
$$

Or equivalently,

$$
u_{i}\left(c_{i}, b_{i}\right)>u_{i}\left(c_{i}^{*}, b_{i}\right)+\varepsilon
$$

Thus $c_{i}^{*} \notin C_{i}^{\varepsilon}\left(\Gamma^{k}\right)$

Part C: Consider some $c_{i}^{*}$ and let $\varepsilon^{*}=\operatorname{Max}\left\{\varepsilon \mid \varepsilon \geq 0, \exists r_{i}\right.$ with $u_{i}\left(r_{i}, b_{i}\right) \geq u_{i}\left(c_{i}^{*}, b_{i}\right)+\varepsilon \quad \forall b_{i} \in$ $\left.B_{i}^{k}\right\}$. Then Show:

1. $\varepsilon^{c_{i}^{*}}\left(\Gamma^{k}\right) \leq \varepsilon^{*}$

Assume $\varepsilon^{c_{i}^{*}}\left(\Gamma^{k}\right)>\varepsilon^{*}$ and so $c_{i}^{*} \notin C_{i}^{\varepsilon^{*}}\left(\Gamma^{k}\right)$. Then part A implies there is some $r_{i} \in$ $\triangle\left(C_{i} \backslash\left\{c_{i}^{*}\right\}\right)$ such that

$$
u_{i}\left(r_{i}, b_{i}\right)>u_{i}\left(c_{i}^{*}, b_{i}\right)+\varepsilon^{*} \quad \text { for all } \quad b_{i} \in B_{i}^{k}
$$

This leads to a contradiction by definition of $\varepsilon^{*}$.

2. $\varepsilon^{c_{i}^{*}}\left(\Gamma^{k}\right) \geq \varepsilon^{*}$

Assume $\varepsilon^{c_{i}^{*}}\left(\Gamma^{k}\right)<\varepsilon^{*}$. So by definition of $\varepsilon^{*}$ and for some $r_{i} \in \Delta\left(C_{i} \backslash\left\{c_{i}^{*}\right\}\right)$,

$$
u_{i}\left(r_{i}, b_{i}\right) \geq u_{i}\left(c_{i}^{*}, b_{i}\right)+\varepsilon^{*} \quad \text { for all } \quad b_{i} \in B_{i}^{k}
$$

So in particular,

$$
u_{i}\left(r_{i}, b_{i}\right)>u_{i}\left(c_{i}^{*}, b_{i}\right)+\varepsilon^{c_{i}^{*}}\left(\Gamma^{k}\right) \quad \text { for all } \quad b_{i} \in B_{i}^{k}
$$

From part b, the latter implies $c_{i}^{*} \notin C_{i}^{\varepsilon_{i}^{c_{i}^{*}}}\left(\Gamma^{k}\right)$ which creates a contradiction by definition of $C_{i}^{\varepsilon}\left(\Gamma^{k}\right)$. Combining parts 1 and 2 proves that $\varepsilon^{c_{i}^{*}}\left(\Gamma^{k}\right)=\varepsilon^{*}$

Proof of Lemma 2.2: Let $\left\{\varepsilon_{-i}^{1}, \ldots, \varepsilon_{-i}^{M}\right\}$ be an ascending ranking of critical epsilons of the set of choices $C_{-i}$, where $M=\left|C_{-i}\right|$. Lemma 2.2 then requires proving two parts: 
(A) Any $b_{i} \in \triangle\left(C_{-i}\right)$ satisfying the finite set of inequalities $b_{i}\left(C_{-i}^{\varepsilon^{m}}\left(\Gamma^{k}\right)\right) \geq \operatorname{Lim}_{\varepsilon \uparrow \varepsilon_{-i}^{m+1}} F_{i}(\varepsilon)$ for all $m \in\{1, \ldots, M-1\}$, also satisfies the infinite set of inequalities of the form $b_{i}\left(C_{-i}^{\varepsilon}\left(\Gamma^{k}\right)\right) \geq F_{i}(\varepsilon)$ for all $\varepsilon \geq 0$.

(B) Any $b_{i} \in \Delta\left(C_{-i}\right)$ satisfying the infinite set of inequalities of the form $b_{i}\left(C_{-i}^{\varepsilon}\left(\Gamma^{k}\right)\right) \geq F_{i}(\varepsilon)$ for all $\varepsilon \geq 0$, also satisfies the finite set of inequalities $b_{i}\left(C_{-i}^{\varepsilon^{m}}\left(\Gamma^{k}\right)\right) \geq \operatorname{Lim}_{\varepsilon \uparrow \varepsilon_{-i}^{m+1}} F_{i}(\varepsilon)$ for all $m \in\{1, \ldots, M-1\}$

Part A: Let $b_{i} \in \Delta\left(C_{-i}\right)$ be some arbitrary belief satisfying $b_{i}\left(C_{-i}^{\varepsilon^{m}}\left(\Gamma^{k}\right)\right) \geq \operatorname{Lim}_{\varepsilon \uparrow \varepsilon_{-i}^{m+1}} F_{i}(\varepsilon)$ for all $m \in\{1, \ldots, M-1\}$. Consider a subset of the infinite set of inequalities $b_{i}\left(C_{-i}^{\varepsilon}\left(\Gamma^{k}\right)\right) \geq F_{i}(\varepsilon)$ for all $\varepsilon \geq 0$ with $\varepsilon \in\left[\varepsilon^{m}, \varepsilon^{m+1}\right)$ for some $m \in\{1, \ldots, M-1\}$.

By definition of $C_{-i}^{\varepsilon}\left(\Gamma^{k}\right)$ and since $\Gamma^{k}$ is finite for any $k \geq 0$,

$$
C_{-i}^{\varepsilon}\left(\Gamma^{k}\right)=C_{-i}^{\varepsilon^{m}}\left(\Gamma^{k}\right) \quad \text { for all } \quad \varepsilon \in\left[\varepsilon^{m}, \varepsilon^{m+1}\right)
$$

So

$$
b_{i}\left(C_{-i}^{\varepsilon}\left(\Gamma^{k}\right)\right) \geq F_{i}(\varepsilon) \quad \text { for all } \quad \varepsilon \in\left[\varepsilon^{m}, \varepsilon^{m+1}\right)
$$

implies,

$$
b_{i}\left(C_{-i}^{\varepsilon^{m}}\left(\Gamma^{k}\right)\right) \geq F_{i}(\varepsilon) \quad \text { for all } \quad \varepsilon \in\left[\varepsilon^{m}, \varepsilon^{m+1}\right)
$$

Since $F_{i}(\varepsilon)$ is weakly increasing in $\varepsilon$, it follows that $b_{i}\left(C_{-i}^{\varepsilon^{m}}\left(\Gamma^{k}\right)\right) \geq \operatorname{Lim}_{\varepsilon \uparrow \varepsilon^{m+1}} F_{i}(\varepsilon)$ implies $b_{i}\left(C_{-i}^{\varepsilon^{m}}\left(\Gamma^{k}\right)\right) \geq F_{i}(\varepsilon)$ for all $\varepsilon \in\left[\varepsilon^{m}, \varepsilon^{m+1}\right)$, and so also implies $b_{i}\left(C_{-i}^{\varepsilon}\left(\Gamma^{k}\right)\right) \geq F_{i}(\varepsilon)$ for all $\varepsilon \in\left[\varepsilon^{m}, \varepsilon^{m+1}\right)$.

Part B: Proving part B is trivial since the finite set of inequalities is by definition of $C_{-i}^{\varepsilon}\left(\Gamma^{k}\right)$ a subset of the infinite set of inequalities. So satisfying the latter automatically implies satisfying the former. 



\section{Chapter 3}

\section{Common Strong Belief in Approximate Rationality}

\subsection{Introduction}

Perfect rationality is a core assumption at the heart of many models examining the reasoning of players in dynamic games. One of the most prominent of such models is Extensive-form Rationalizability (Pearce 1984), epistemically characterized by rationality and Common Strong Belief in Rationality (CSBR) (Battigalli and Siniscalchi 2002) in complete type space ${ }^{1}$. Player $i$ strongly believes in his opponents' rationality at a certain information set $h$ in the game, if player $i$ assigns at $h$ probability 1 to the set of opponents' rational strategy combinations leading to that information set, as long as such set is non-empty. If this holds for all information sets at which player $i$ is active, that player is said to strongly believe in his opponents' rationality. CSBR models the reasoning of players in a dynamic game in which each player strongly believes in his opponents' rationality, strongly believes his opponents are rational and strongly believe in their opponents' rationality, and so on.

However the application of CSBR to some well known games yields theoretical predictions that often come at odds with experimentally observed outcomes. An example of such games is the Centipede game (Rosenthal 1981; Binmore 1987), where the only possible outcome of the game under CSBR is the backward induction one in which the first player takes all at the first possible chance. Experimental evidence, on the other hand, has often sharply diverted from such theoretical prediction (see for example McKelvey and Palfrey 1992; Fey et al. 1996). One explanation deemed plausible is that players allow for the possibility that their opponents make mistakes with that being commonly believed by the players (Rosenthal, 1981). In this paper, we formalize this interpretation by introducing Common Strong Belief in $F$-Rationality (CSB $F \mathrm{R}$ )

\footnotetext{
${ }^{1}$ Perea(2012) shows that Extensive-form Rationalizability tcan also be epistemically characterized by rationality and Common Strong Belief in Rationality in epistemic models that are sufficiently rich.
} 
as a generalization of CSBR. While preserving the essence of CSBR, our CSBF R allows players to believe their opponents may make mistakes. Under CSBFR players are assumed to be approximately rational, with the specific form of approximate rationality being $F$-rationality (Mounir, Perea and Tsakas (MPT) 2013).

The core idea of $F$-rationality has two main components; $\varepsilon$-rationality of players and the uncertainty on each player's part about the extent of his opponents' irrationality. Firstly, players believe their opponents to be $\varepsilon$-rational in the sense of Radner (1980). Thus any player $j$ believes his opponents $i \neq j$ may each sacrifice up to $\varepsilon_{i}$ in expected utility in response to the beliefs they holds about their opponents' strategy choices. The term $\varepsilon_{i}$ can be thought of as the margin of error, or degree of irrationality, of player $i$. Such margin of error $\varepsilon_{i}$, is a characteristic of the player and is each player $i$ 's private information. Each player's actual margin of error $\varepsilon_{i}$ will thus play no role in our model or in the steps of the procedure we design. Instead, focus will be on what each player believes his opponents' margin of error could be, which leads us to the second component of $F$-rationality.

Every player $i$ holds some weakly increasing function $F_{i}:[0, \infty) \rightarrow[0,1]$ which represents a lower bound to the probability player $i$ can assign to each of his opponents' possible extents of irrationality, or potential values of $\varepsilon$. Player $i$ assigns a probability of at least $F_{i}(\varepsilon)$ to the event of his opponent having a margin of error of $\varepsilon$ or less, for every possible level $\varepsilon \geq 0$. Functions $F_{i}$ do not change throughout the game and are assumed to be transparent with all players expressing common belief therein. Thus $F$-Rationality can be considered a generalization of Radner's $\varepsilon$-rationality where the assumption of the transparency of players' $\varepsilon$ is relaxed. Note that $F_{i}$ could, but need not be, a cumulative probability distribution.

Functions $F_{i}$ specify a minimum bound to the probability that each player $i$ must assign to every potential level of his opponents' rationality. For every possible level of $\varepsilon \geq 0$ there is for every player $i$ a corresponding set of strategies that could be $\varepsilon$-rationally made for some belief about the opponents' strategies. The minimum bounds on probabilities assigned to different levels of $\varepsilon$ can then be translated into restrictions on the probabilities player $i$ can assign to different sets of his opponents' strategy combinations. The smaller the $\varepsilon$ associated with a given set of strategy combinations, the higher the upper bound to the probability that could be assigned to it.

The basic restriction in our model of dynamic games is strong belief in $F$-rationality. Strong belief in $F$-rationality requires player $i$ to assign at the beginning of the game a minimum bound of $F_{i}(\varepsilon)$ to the event of his opponents having an error margin of at most $\varepsilon$. Furthermore, strong belief in $F$-rationality requires every player $i$ to respect this restriction at information sets other than the beginning of the game whenever that is possible. Establishing common belief 
in such restriction, our model defines common strong belief in $F$-rationality (CSBFR). This is done using an epistemic model. An epistemic model assigns to every player a set of types each of which encodes a conditional belief hierarchy the player could hold about his opponents' strategies and conditional beliefs. Moreover, an iterative procedure is designed to characterize exactly those first order conditional beliefs that an epistemic type satisfying CSBFR can hold. The links of both the epistemic model and the iterative procedure to Common Strong Belief in Rationality and Iterated Conditional Dominance procedure, respectively, are highlighted. Furthermore, as an illustrative example, the iterative procedure is applied to the Centipede game examined in McKelvey and Palfery (1992).

The paper is structured as follows. Section 3.4 formalizes the concept of Common Strong Belief in $F$-rationality. Section 3.3 proceeds to design the recursive procedure of Iterated Elimination of $F$-Dominated Conditional Beliefs, that characterizes exactly those beliefs that can be held by an epistemic type expressing common strong belief in $F$-rationality. Furthermore, in Section 3 , a well known version of the Centipede game is used to provide an example that further illustrates the steps of the recursive procedure. Section 3.4 summarizes and discusses the results we obtained for the Centipede game under common strong belief in $F$-rationality.

\subsection{Common Strong Belief in $F$-Rationality}

In this section we introduce Common Strong Belief in F-Rationality as a model of the reasoning of players in dynamic games. The section starts with some notations and a definition of the basic framework our model will be built into, namely a countable epistemic model. Subsection 3.2.1 covers in greater detail the specific form of approximate rationality of interest in this paper and formalizes the notion of strong belief in the opponents' $F$-rationality. Subsection 3.2.3 defines $k$-fold strong belief in $F$-rationality as well as common strong belief in $F$-rationality. The latter also shows CSBR to be a special case of CSBFR. Finally, subsection 3.2.4 presents an example of an epistemic model with types expressing various degrees of strong belief in $F$-rationality.

\subsubsection{Notation and Basic Model}

Let $\Gamma=\left\{\mathcal{X}, \mathcal{Z}, \mathcal{I},\left(u_{i}\right)_{i \in \mathcal{I}}\right\}$ be a finite dynamic game with complete information, in which players are assumed to have perfect recall. Let $\mathcal{X}$ be the set of non-terminal histories of the game, $\mathcal{Z}$ be the set of terminal histories, $\mathcal{I}$ be the set of players and $u_{i}: \mathcal{Z} \rightarrow \mathfrak{R}$ be the utility function of player $i \in \mathcal{I}$. Let $\mathcal{H}$ be the set of all information sets of the game, where every $h \in \mathcal{H}$ corresponds to some subset of non-terminal histories $\mathcal{X}$. Define $\mathcal{H}_{i}$ to be the set of information 
sets where player $i \in \mathcal{I}$ is active, then $\mathcal{H}:=\cup_{i \in \mathcal{I}} \mathcal{H}_{i}$. Furthermore, let the information set $\emptyset$ be the auxiliary history before any player $i$ moves. For every player $i$ let the set $\mathcal{H}_{i}^{*}=\mathcal{H}_{i} \cup\{\emptyset\}$.

The set $C_{i}(h)$ is the set of choices or actions available to player $i$ at information set $h \in \mathcal{H}_{i}$ at which player $i$ is active. A strategy $s_{i}$ of player $i$ is a complete action plan assigning an action $s_{i}(h) \in C_{i}(h)$ to every information set $h \in \mathcal{H}_{i}$ it reaches according to the series of choices constituting $s_{i}$. Furthermore, $S_{i}$ is the set of all strategies available to player $i$. Let $S_{i}(h)$ be the set of strategies of player $i$ leading to information set $h$, i.e. $S_{i}(h)=\left\{s_{i} \in\right.$ $S_{i} \mid$ there exists $s_{-i}$ where $\left(s_{i}, s_{-i}\right)$ leads to $\left.h\right\}$, where $s_{-i}=\left(s_{1}, \ldots, s_{i-1}, s_{i+1}, \ldots, s_{n}\right) \in \prod_{j \neq i} S_{j}$. Similarly, $S_{-i}(h)=\left\{s_{-i} \in S_{-i} \mid\right.$ there exists $s_{i}$ where $\left(s_{i}, s_{-i}\right)$ leads to $\left.h\right\}$, where $S_{-i}=\prod_{j \neq i} S_{j}$. Also let $\mathcal{H}_{i}\left(s_{i}\right) \subseteq \mathcal{H}_{i}$ denote the set of information sets reached by strategy $s_{i}$, i.e. $\mathcal{H}_{i}\left(s_{i}\right):=$ $\left\{h \in \mathcal{H}_{i} \mid s_{i} \in S_{i}(h)\right\}$. Thus we follow Rubinstein (1991) in defining a strategy $s_{i}$ as a plan of action of player $i$, that assigns to every $h \in \mathcal{H}_{i}\left(s_{i}\right)$ a choice $s_{i}(h) \in C_{i}(h)$.

We introduce $\mathrm{CSB} F \mathrm{R}$ within the context of an epistemic model. Let $M$ be an epistemic model assigning to each player $i$ a countable set of types $T_{i}$. Epistemic types provide a convenient way to model players' belief hierarchies. Any epistemic type $t_{i} \in T_{i}$ implies for every information set $h \in \mathcal{H}_{i}^{*}$ a belief for player $i$ about opponents' strategy combinations $s_{-i}$, a belief about opponents' beliefs about $i$ 's own strategies, player $i$ 's belief about opponents' beliefs about $i$ 's beliefs, and so forth. Definition 4.2.2 formally introduces our epistemic model.

Definition 3.1 Let $\Gamma$ be a dynamic game. The tuple $M=\left(T_{i}, b_{i}\right)_{i \in \mathcal{I}}$ is a countable epistemic model for game $\Gamma$, where

- $T_{i}$ is a countable set of types for player $i$

- Function $b_{i}: \mathcal{H}_{i}^{*} \rightarrow \Delta\left(S_{-i}(h) \times T_{-i}\right)$ assigns to every information set $h \in \mathcal{H}_{i}^{*}$ of player $i$ a probability distribution $b_{i}\left(t_{i}, h\right) \in \Delta\left(S_{-i}(h) \times T_{-i}\right)$ over opponents' strategy-type combinations leading to information set $h$.

So every type $t_{i} \in T_{i}$ holds a conditional belief vector $b_{i}\left(t_{i}\right)=\left(b_{i}\left(t_{i}, h\right)\right)_{h \in \mathcal{H}_{i}^{*}}$, where $b_{i}\left(t_{i}, h\right) \in$ $\triangle\left(S_{-i}(h) \times T_{-i}\right)$ is a probability distribution over opponents' strategy- type combinations $\left(S_{-i}(h) \times\right.$ $T_{-i}$ ) leading to information set $h$. Each $t_{i} \in T_{i}$ thus induces an infinite conditional belief hierarchy, where $b_{i}^{1}\left(t_{i}\right)=\left(b_{i}^{1}\left(t_{i}, h\right)\right)_{h \in \mathcal{H}_{i}^{*}}$ constitutes the first order conditional belief vector held by $t_{i}$. Hence, for every information set $h \in \mathcal{H}_{i}^{*}$,

$$
b_{i}^{1}\left(t_{i}, h\right)\left(s_{-i}\right)=\sum_{t_{-i} \in T_{-i}} b_{i}\left(t_{i}, h\right)\left(s_{-i}, t_{-i}\right) \text { for every } s_{-i} \in S_{-i}(h) .
$$


The first order belief of any type $t_{i}$ is thus a probability distribution about opponents' strategy combinations, i.e. $b_{i}^{1}\left(t_{i}, h\right) \in \Delta\left(S_{-i}(h)\right)$. Higher order beliefs, on the other hand, are probability distributions over opponents' beliefs or type-combinations $T_{-i}$ as well as strategies.

\subsubsection{Strong Belief in $\boldsymbol{F}$-Rationality}

Player $i$ assigns a probability of at least $F_{i}(\varepsilon)$ to the event of his opponents having a margin of error of $\varepsilon$ or less, for every possible level of such margin $\varepsilon \geq 0$ and for some weakly increasing function $F_{i}:[0, \infty) \rightarrow[0,1]$. The lower bound of the probability assigned to each potential level of opponents' rationality $\varepsilon \geq 0$, can be translated into probabilities assigned to opponents' strategy-type combinations $\prod_{j \neq i}\left(S_{j} \times T_{j}\right)$. This requires defining $\varepsilon$-rational strategytype combinations $\left(S_{j} \times T_{j}\right)^{\varepsilon}$ for every player $j$, which is the set of strategy-type combinations of player $j$ where the strategy $s_{j}$ is $\varepsilon$-optimal for the first order belief $b_{j}^{1}\left(t_{j}\right)$ held by the type $t_{j}$. The set $\left(S_{j} \times T_{j}\right)^{\varepsilon}$ is formally defined in Definition 3.2.

Definition 3.2 The set $\left(S_{j} \times T_{j}\right)^{\varepsilon}$ of player $j$ is the set of strategy-type combinations $\left(s_{j}, t_{j}\right) \in$ $\left(S_{j} \times T_{j}\right)$ where $s_{j}$ is $\varepsilon$-optimal for type $t_{j}$, i.e. where for every $h \in \mathcal{H}_{j}^{*}\left(s_{j}\right)$ the following condition is satisfied

$$
u_{j}\left(s_{j}^{\prime}, b_{j}^{1}\left(t_{j}, h\right)\right) \leq u_{j}\left(s_{j}, b_{j}^{1}\left(t_{j}, h\right)\right)+\varepsilon \quad \text { for all } s_{j}^{\prime} \in S_{j}(h)
$$

A strategy $s_{j}$ is thus $\varepsilon$-optimal for the first order belief $b_{j}^{1}\left(t_{j}\right)$ of type $t_{j}$, if its expected utility given $b_{j}^{1}\left(t_{j}\right)$ is always within $\varepsilon$ of that of all other strategies $S_{j}(h) \backslash\left\{s_{j}\right\}$ at every information set $h \in \mathcal{H}_{j}^{*}\left(s_{j}\right)$. Define for every $\varepsilon \geq 0$ the set $\left(S_{-i} \times T_{-i}\right)^{\varepsilon}$ to be $\varepsilon$-rational strategy-type combinations of player $i$ 's opponents, i.e.

$$
\left(S_{-i} \times T_{-i}\right)^{\varepsilon}=\prod_{j \neq i}\left(S_{j} \times T_{j}\right)^{\varepsilon} \quad \text { for every } \varepsilon \geq 0 .
$$

At the beginning of the game, type $t_{i}$ of player $i$ believes his opponents are $\varepsilon$-rational for some possibly unknown value(s) of $\varepsilon$, and uses the transparent $F_{i}$ as a lower bound for the probability to be assigned to the set of opponents' $\varepsilon$-rational strategy-type combinations $\left(S_{-i} \times T_{-i}\right)^{\varepsilon}$ for every $\varepsilon \geq 0$. Type $t_{i}$ is said to respect the F-condition, if the belief held by $t_{i}$ at $\emptyset, b_{i}\left(t_{i}, \emptyset\right)$ assigns at least $F_{i}(\varepsilon)$ to the set $\left(S_{-i} \times T_{-i}\right)^{\varepsilon}$ for every $\varepsilon \geq 0$, i.e.

$$
b_{i}\left(t_{i}, \emptyset\right)\left(S_{-i} \times T_{-i}\right)^{\varepsilon} \geq F_{i}(\varepsilon) \text { for all } \varepsilon \geq 0 .
$$


As the game proceeds, type $t_{i}$ continues to respect the $F$-condition at every following information set $h \in \mathcal{H}_{i}$ as long as this is possible. Conditional beliefs $b_{i}\left(t_{i}, h\right)$ are, therefore, the Bayesian updating of some $b_{i}\left(t_{i}^{\prime}, \emptyset\right)$ that satisfies the $F$-condition, as long as such Bayesian updating is possible. Note that $b_{i}\left(t_{i}^{\prime}, \emptyset\right)$ may or may not coincide with $b_{i}\left(t_{i}, \emptyset\right)$. Furthermore, take two information sets $h$ and $h^{\prime}$ of player $i$, then $b_{i}\left(t_{i}, h\right)$ and $b_{i}\left(t_{i}, h^{\prime}\right)$ need not be the Bayesian updating of the same $b_{i}\left(t_{i}^{\prime}, \emptyset\right)$. Type $t_{i}$ described above is said to strongly believe in opponents' $F$-rationality. Definition 3.3 formalizes this notion further.

Definition 3.3 A type $t_{i}$ strongly believes in the opponents' $F$-rationality, denoted $t_{i} \in F R_{i}$, if it satisfies the following conditions:

1. The F-condition: $b_{i}\left(t_{i}, \emptyset\right)\left(S_{-i} \times T_{-i}\right)^{\varepsilon} \geq F_{i}(\varepsilon)$ for all $\varepsilon \geq 0$

2. At every $h \in \mathcal{H}_{i}$, if there exists some $t_{i}^{\prime}$ satisfying condition 1 with $b_{i}\left(t_{i}^{\prime}, \emptyset\right)\left(S_{-i}(h) \times T_{-i}\right)>0$, then $b_{i}\left(t_{i}, h\right)$ is the Bayesian updating of one such $b_{i}\left(t_{i}^{\prime}, \emptyset\right)$. If there exists no such $b_{i}\left(t_{i}^{\prime}, \emptyset\right)$, no further restrictions are to be imposed on $b_{i}\left(t_{i}, h\right)$.

Thus strongly believing in the opponents' $F$-rationality, a player of type $t_{i}$ assigns at the beginning of the game a probability of at least $F_{i}(\varepsilon)$ to the event of his opponents' having margins of error of at most $\varepsilon$. In other words, type $t_{i}$ satisfies the $F$-condition. Type $t_{i}$ then maintains the $F$-condition as long as possible, as part two of Definition 3.3 implies. Note that the latter is, to our knowledge, new to the literature. The second part of Definition 3.3 is an intermediate case between requiring conditional beliefs to satisfy Bayesian updating on the one hand and not imposing any restrictions on updating on the other hand. The reason such construction has been chosen for, is to guarantee that the F-condition is satisfied and at the same time allow the players' belief updating method to deviate from Bayesian updating. In other words, our model recognizes that players might change their beliefs as the game progresses.

\subsubsection{Common Strong Belief in $F$-Rationality}

A type $t_{i}$ strongly believing in the opponents' $F$-rationality is said to express 1 -fold strong belief in $F$-rationality, denoted by $t_{i} \in T_{i}^{1}$. Type $t_{i}$ expresses up to 2 -fold strong belief in $F$-rationality if it strongly believes in the opponents' $F$-rationality and whenever possible assigns positive probability only to opponents' type combinations $t_{-i} \in T_{-i}^{1}$ expressing 1-fold strong belief in $F$-rationality. More generally, type $t_{i}$ expresses up to $k$-fold strong belief in $F$-rationality for $k \geq 2$, i.e $t_{i} \in T_{i}^{k}$, if it expresses up to $k-1$ - fold strong belief in $F$-rationality and assigns positive probability only to opponents' type combinations $t_{-i} \in T_{-i}^{k-1}$ expressing up to $k-1$-fold strong belief in $F$-rationality whenever this is possible. Moreover, $t_{i}$ expresses common strong 
belief in $F$-rationality if it expresses up to $k$-fold strong belief in $F$-rationality for every $k \geq 1$. Definition 3.4 below formally introduces this.

Definition 3.4 Types expressing 1-fold strong belief in F-rationality are defined by:

$$
T_{i}^{1}:=\left\{t_{i} \in T_{i} \mid t_{i} \in F R_{i}\right\}
$$

Moreover, types expressing up to $k$-fold strong belief in $F$-rationality for $k \geq 2$, and those expressing common strong belief in $F$-rationality can be defined recursively as follows:

$$
\begin{gathered}
T_{i}^{k}:=\left\{t_{i} \in T_{i}^{k-1} \mid b_{i}\left(t_{i}, h\right)\left(S_{-i}(h) \times T_{-i}^{k-1}\right)=1 \quad \forall h \in \mathcal{H}_{i}^{*} \text { whenever possible }\right\} \\
T_{i}^{\infty}:=\bigcap_{k=1}^{\infty} T_{i}^{k}
\end{gathered}
$$

Because of the fact that a countable epistemic model does not induce all possible belief hierarchies, we need to impose a richness condition model $M$ needs to satisfy similarly to Perea (2012). Fix some $k \geq 1$ and consider some $h \in \mathcal{H}_{i}$, at which there is no $b_{i}\left(t_{i}^{\prime}, \emptyset\right)$ satisfying the $F$-condition with $b_{i}\left(t_{i}^{\prime}, \emptyset\right)\left(S_{-i}(h) \times T_{-i}^{k-1}\right)>0$. By Definitions 3.3 and 3.4, one would then conclude that no further restrictions are to be imposed on $b_{i}\left(t_{i}, h\right)$ for $t_{i}$ to express up to $k$-fold strong belief in $F$-rationality. There may, however; exist such a type $t_{i}^{\prime}$ outside $M$. This is an example of a problem that could arise if the epistemic model is insufficiently rich. Following Perea (2012), sufficient richness can be guaranteed in our countable epistemic model if the conditions in Definition 3.5 are met.

Definition 3.5 The countable epistemic model $M$ is said to be sufficiently rich if the following holds for every $h \in \mathcal{H}_{i}$ and for every player $i \in \mathcal{I}$ :

- For $k=1$ : If there exist some $t_{i}^{\prime}$, possibly outside $M$, satisfying the $F$ condition for which $b_{i}\left(t_{i}^{\prime}, \emptyset\right)\left(S_{-i}(h) \times T_{-i}\right)>0$, then M must contain at least one such $t_{i}^{\prime}$.

- For $k>1$ : If there exist some $t_{i}^{\prime}$, possibly outside $M$, satisfying the $F$ condition for which $b_{i}\left(t_{i}^{\prime}, \emptyset\right)\left(S_{-i}(h) \times T_{-i}^{k-1}\right)>0$, then $M$ must contain at least one such type $t_{i}^{\prime}$.

Notably, Common Strong Belief in F-Rationality serves as a generalization of the established Common Strong Belief in Rationality (Battigalli and Siniscalchi 2002). A type $t_{i}$ is said to express up to $k$-fold strong belief in rationality for some $k \geq 1$, if at every $h \in \mathcal{H}_{i}^{*}$ the following 
holds: whenever there exist some opponents' strategy-type combinations $\left(s_{-i}, t_{-i}\right)$, where for every $j \neq i s_{j} \in S_{j}(h)$, type $t_{j}$ expresses up to $k-1$-fold belief in rationality and $s_{j}$ is optimal for $t_{j}$, type $t_{i}$ must assign positive probability only to such combinations. Definition 3.6 formalizes the condition of $k$-fold strong belief in rationality in our notation.

Definition 3.6 Type $t_{i}$ expresses 1-fold strong belief in rationality if for every $h \in \mathcal{H}_{i}^{*}$, $b_{i}\left(t_{i}, h\right)\left(S_{-i}(h) \times T_{-i}\right)^{0}=1$, whenever the set $\left(S_{-i}(h) \times T_{-i}\right)^{0}$ is non-empty, and where $\left(S_{-i}(h) \times\right.$ $\left.T_{-i}\right)^{\varepsilon}=\left\{\left(s_{-i}, t_{-i}\right) \in\left(S_{-i} \times T_{-i}\right)^{\varepsilon} \mid s_{-i} \in S_{-i}(h)\right\}$.

Type $t_{i}$ expresses up to $k$-fold strong belief in rationality if for every $h \in \mathcal{H}_{i}^{*}, b_{i}\left(t_{i}, h\right)\left(S_{-i}(h) \times\right.$ $\left.T_{-i}^{k-1}\right)^{0}=1$, whenever the set $\left(S_{-i}(h) \times T_{-i}^{k-1}\right)^{0}$ is non-empty, and where $\left(S_{-i}(h) \times T_{-i}^{k-1}\right)^{\varepsilon}=$ $\left\{\left(s_{-i}, t_{-i}\right) \in\left(S_{-i} \times T_{-i}\right)^{\varepsilon} \mid s_{-i} \in S_{-i}(h)\right.$ and $\left.t_{-i} \in T_{-i}^{k-1}\right\}$.

Theorem 3.1 below shows that Common Strong Belief in Rationality is a special case of Common Strong Belief in $F$-Rationality. Type $t_{i}$ expresses up to $k$-fold strong belief in rationality if it expresses up to $k$-fold strong belief in $F$-rationality for one particular form of function $F_{i}$. Furthermore, any $t_{i}$ expressing up to $k$-fold strong belief in rationality can also be shown to express up to $k$-fold strong belief in $F$-rationality for that particular form of $F_{i}$.

Theorem 3.1 If $F_{i}(\varepsilon)=1$ for all $\varepsilon \geq 0$ and for all $i \in \mathcal{I}$, then a type $t_{i}$ expresses up to $k$-fold strong belief in $F$-rationality if and only if it expresses up to $k$-fold strong belief in rationality.

Common Strong Belief in $F$-Rationality is also an extension of Common Belief in $F$-Rationality (MPT 2013) to the case of dynamic games. If the set of information sets $\mathcal{H}$ is a singleton, conditions a type needs to satisfy in order to strongly believe in the opponents' $F$-rationality reduce to the $F$-condition (condition 1 in Definition 2.3). The latter exactly define types believing in the opponents' $F$-rationality (Definition 2 in MPT 2013). Definition 3.4 then becomes identical to Definition 3 in MPT 2013. Thus if $\mathcal{H}$ is a singleton, Common Strong Belief in $F$-Rationality reduces to Common Belief in $F$-Rationality.

\subsubsection{An Example}

This section uses an example of a simple epistemic model consisting of one type for each player, to better illustrate strong belief in $F$-rationality. Consider the Centipede game with exponentially growing payoffs of Figure 1, borrowed from Mckelvey and Palfrey (1992). 


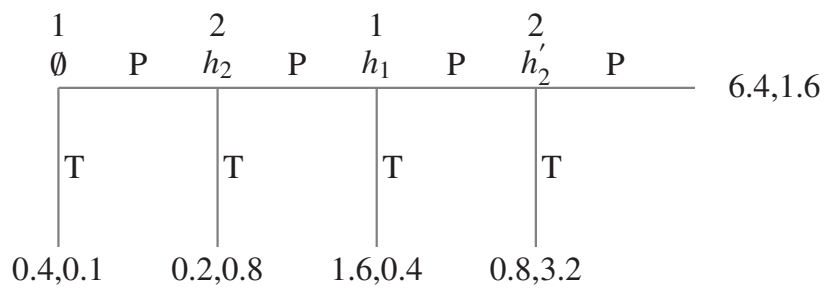

"Figure 1: Centipede game from Mckelvey and Palfrey 1992"

Moreover, consider two types $t_{1}$ and $t_{2}$ in some countable epistemic model $M$ as in Table 1 below. Let $F_{i}$ be for both $i$ as follows

$$
F_{i}(\varepsilon)= \begin{cases}\frac{\varepsilon}{3.2} & \text { if } \varepsilon \leq 3.2 \\ 1 & \text { if } \varepsilon \geq 3.2\end{cases}
$$

It can be shown that type $t_{1}$ in Table 1 below strongly believes in player 2 's $F$-rationality.

\begin{tabular}{c|cc}
\hline & Player 1 & Player 2 \\
\hline Types & $T_{1}=\left\{t_{1}\right\}$ & $T_{2}=\left\{t_{2}\right\}$ \\
\hline Beliefs & $b_{1}\left(t_{1}, \emptyset\right)=0.75\left(T, t_{2}\right)+0.25\left(P P, t_{2}\right)$ & $b_{2}\left(t_{2}, \emptyset\right)=\left(T, t_{1}\right)$ \\
& $b_{1}\left(t_{1}, h_{1}\right)=\left(P T, t_{2}\right)$ & $b_{2}\left(t_{2}, h_{2}\right)=\left(P T, t_{1}\right)$ \\
& & $b_{2}\left(t_{2}, h_{2}^{\prime}\right)=\left(P P, t_{1}\right)$ \\
\hline
\end{tabular}

"Table 1: Finite Epistemic Model M"

Under the belief held by type $t_{2}$, strategy $P P$ is a best response to $b_{2}^{1}\left(t_{2}, \emptyset\right)$, is within 0.4 in expected utility of the best response to $b_{2}^{1}\left(t_{2}, h_{2}\right)$ and within 1.6 in expected utility of the best response to $b_{2}^{1}\left(t_{2}, h_{2}^{\prime}\right)$. Strategy $P P$ is, therefore, 1.6-optimal for $b_{2}^{1}\left(t_{2}\right)$. Given the form of $F_{1}$, player 1 cannot assign more than 0.5 to the event of player 2 having a margin of error that is 1.6 or higher. Type $t_{1}$ assigns at the beginning of the game $\emptyset$ probability 0.25 to the 1.6-optimal $\left(P P, t_{2}\right)$ and 0.75 to the 0 -optimal $\left(T, t_{2}\right)$. Type $t_{1}$ thus assigns at least $F_{1}(\varepsilon)$ to the event of player 2 being $\varepsilon$-rational for every $\varepsilon \geq 0$, thereby satisfying the $F$-condition. Moreover, $b_{1}\left(t_{1}, h_{1}\right)$ is the Bayesian updating of the belief $b_{1}(\cdot, \emptyset)=0.25\left(T, t_{2}\right)+0.75\left(P T, t_{2}\right)$. Since $P T$ is 0.4-optimal for $t_{2}$, it can be concluded that $b_{1}(\cdot, \emptyset)=0.25\left(T, t_{2}\right)+0.75\left(P T, t_{2}\right)$ satisfies the $F$-condition. Type $t_{1}$ thus strongly believes in player 2's $F$-rationality. Types $t_{2}$, on the other hand, does not strongly believe in player 1 's $F$-rationality, as $b_{2}\left(t_{2}, \emptyset\right)$ assigns probability 1 to $\left(T, t_{1}\right)$ while $T$ is only 1.35 -optimal for $b^{1}\left(t_{1}\right)$, i.e. $t_{2}$ does not meet the $F$-condition. 


\subsection{Iterated Elimination of $F$-Dominated Conditional Beliefs}

Now that the model has been constructed, we design the procedure of Iterated Elimination of $F$-Dominated Conditional Beliefs to characterize these first order beliefs that can be held by an epistemic type expressing up to $k$-fold strong belief in $F$-rationality for any $k \geq 1$. The basic idea of the procedure is similar to that of the procedure of "Iterated Elimination of $F$-Dominated Beliefs" in MPT (2013). Subsection 3.3.1 formalizes the procedure and explains its steps. Subsection 3.3.2 stresses some practical matters regarding the implementation of the procedure. Finally, subsection 3.3.3 applies the procedure to an example.

\subsubsection{The Procedure}

Prior to introducing the steps of the procedure, the notions of feasible belief sets and decision problem need to be defined. Under 0 -fold strong belief in $F$-rationality, each player can hold any first order belief about opponents' strategies. Thus before the procedure starts, all sets

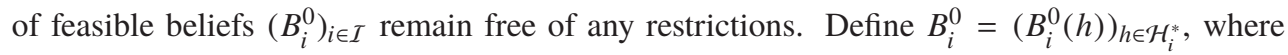
$B_{i}^{0}(h)=\Delta\left(S_{-i}(h)\right)$ for all $h \in \mathcal{H}_{i}$ and where $B_{i}^{0}(\emptyset)=\Delta\left(S_{-i}\right)$. Let $B_{i}^{k}(h)$ be the set of first order beliefs of player $i$ at information set $h \in \mathcal{H}_{i}^{*}$ after round $k$ of the procedure. Moreover, $B_{i}^{k}=\left(B_{i}^{k}(h)\right)_{h \in \mathcal{H}_{i}^{*}}$.

All players start off facing the game $\Gamma$. So before the procedure begins, each player $i$ faces various decision problems $\Gamma_{i}^{0}(h)$, each corresponding to one of his information sets $\mathcal{H}_{i}^{*}$. Define $\Gamma_{i}^{0}(h)=\left(S_{i}(h), \Delta\left(S_{-i}(h)\right)\right)$ for all $h \in \mathcal{H}_{i}$ and $\Gamma_{i}^{0}(\emptyset)=\left(S_{i}, \Delta\left(S_{-i}\right)\right)$. This can be rewritten in terms of belief sets as $\Gamma_{i}^{0}(h)=\left(S_{i}(h), B_{i}^{0}(h)\right)$ for all $h \in \mathcal{H}_{i}^{*}$. The decision problems of player $i$ after round $k$ of the procedure, for $k \geq 0$ can be defined by; $\Gamma_{i}^{k}(h)=\left(S_{i}(h), B_{i}^{k}(h)\right)$ for all $h \in \mathcal{H}_{i}^{*}$. Note that $S_{i}$ remains unchanged throughout the rounds of the procedure. This is due to the fact that the procedure always eliminates beliefs and never strategies.

Recall that for player $i$ to strongly believe in his opponents' $F$-rationality, he must assign at the beginning of the game a probability of at least $F_{i}(\varepsilon)$ to the event of his opponents' having a margin of error of at most $\varepsilon$ (Condition 1 in Definition 3.3). Believing in the opponents' $F$-rationality, type $t_{i}$ assigns at the beginning of the game a probability of at least $F_{i}(\varepsilon)$ to the set $\left(S_{-i} \times T_{-i}\right)^{\varepsilon}=\underset{j \neq i}{\times}\left(S_{j} \times T_{j}\right)^{\varepsilon}$ for every $\varepsilon \geq 0$. For any strategy $s_{j}$ to make it in combination with some type $t_{j}$ into set $\left(S_{j} \times T_{j}\right)^{\varepsilon}$ for some $\varepsilon \geq 0$, strategy $s_{j}$ has to be $\varepsilon$-optimal for the first order belief held by $t_{j}$. Strategy $s_{j}$ would thus never appear in $\left(S_{j} \times T_{j}\right)^{\varepsilon}$ for some $\varepsilon$ in combination with any type $t_{j}$, if there is no first order belief for which $s_{j}$ is $\varepsilon$-optimal. 
The $F$-condition can be translated into probability distributions over opponents' strategy combinations, by assigning at least $F_{i}(\varepsilon)$ to opponents' strategy combinations that are $\varepsilon$-optimal for some belief. Define $S_{i}^{\varepsilon}\left(B_{i}^{k}\right)$ to be the set of strategies of player $i$ that are $\varepsilon$-optimal for some belief in $B_{i}^{k}$. In other words, $S_{i}^{\varepsilon}\left(B_{i}^{k}\right)$ is the set of strategies of player $i$ that are $\varepsilon$-rational in the decision problem $\Gamma_{i}^{k}$, i.e. $S_{i}^{\varepsilon}\left(B_{i}^{k}\right)=S_{i}^{\varepsilon}\left(\Gamma_{i}^{k}\right)$.

Definition 3.7 Let the set $S_{i}^{\varepsilon}\left(B_{i}^{k}\right)$ be defined as follows

$$
S_{i}^{\varepsilon}\left(B_{i}^{k}\right)=\left\{s_{i} \in S_{i} \mid \exists b_{i} \in B_{i}^{k} \text { s.t. } u_{i}\left(s_{i}, b_{i}(h)\right) \geq u_{i}\left(s_{i}^{\prime}, b_{i}(h)\right)-\varepsilon \forall s_{i}^{\prime} \in S_{i}(h), \forall h \in \mathcal{H}_{i}^{*}\left(s_{i}\right)\right\}
$$

A first order belief $b_{i}(\emptyset) \in B_{i}^{1}(\emptyset)$ is consistent with strong belief in opponents' $F$-rationality if at the beginning of the game it assigns a probability of at least $F_{i}(\varepsilon)$ to the $\operatorname{set}^{2} S_{-i}^{\varepsilon}\left(B_{-i}^{0}\right)$, where $B_{i}^{1}(\emptyset)$ is the set of beliefs of player $i$ at the beginning of the game that survive the first round of the procedure (see condition 1.1 below).

Strong belief in $F$-rationality requires player $i$ to believe in opponents' $F$-rationality whenever possible. To preserve the essence of belief in $F$-rationality captured by condition 1.1 of the procedure, first order beliefs surviving the procedure at information sets $h \neq \emptyset$ are defined to be the Bayesian updates of some belief satisfying condition 1.1, whenever there exists such belief by which information set $h$ is reached with positive probability. Elements of the belief set $B_{i}^{1}(h)$ are thus the Bayesian update of some $b_{i}(\emptyset) \in B_{i}^{1}(\emptyset)$, whenever there exist at least some $b_{i}(\emptyset) \in B_{i}^{1}(\emptyset)$ with $b_{i}(\emptyset)\left(S_{-i}(h)\right)>0$. Assuming the latter condition is satisfied, take some $b_{i}(\emptyset) \in B_{i}^{1}(\emptyset) \subseteq \Delta\left(S_{-i}\right)$, the Bayesian updating of $b_{i}(\emptyset)$ at $h$ for all $s_{-i} \in S_{-i}(h)$ then is

$$
b_{i}(h)\left(s_{-i}\right)=\frac{b_{i}(\emptyset)\left(s_{-i}\right)}{b_{i}(\emptyset)\left(S_{-i}(h)\right)} .
$$

Because every element $b_{i}(h) \in B_{i}^{1}(h)$, for $h \neq \emptyset$, is the Bayesian updating of some element $b_{i}(\emptyset) \in B_{i}^{1}(\emptyset)$, we refer to $B_{i}^{1}(h)$ as the Bayesian updating of $B_{i}^{1}(\emptyset)$ (see steps 1.2 and 2.2 below). If, on the other hand, $b_{i}(\emptyset)\left(S_{-i}(h)\right)=0$ for all $b_{i}(\emptyset) \in B_{i}^{1}(\emptyset)$, then information set $h$ cannot be reached with positive probability under strong belief in $F$-rationality for the specific functions $\left(F_{i}\right)_{i \in \mathcal{I}}$. In that case, no further restrictions are imposed on player $i$ 's feasible belief set at $h$ and so $B_{i}^{1}(h)=B_{i}^{0}(h)$, i.e $B_{i}^{1}(h)=\Delta\left(S_{-i}(h)\right)$. The definition of $\left(B_{i}^{1}(h)\right)_{h \in \mathcal{H}_{i}^{*}}$ for all $i \in \mathcal{I}$ concludes round 1 of the procedure. Further rounds of the procedure follow the same steps explained above, with each new round $k$ being based on a new set of decision problems $\left(\left(\Gamma_{i}^{k-1}(h)\right)_{h \in \mathcal{H}_{i}^{*}}\right)_{i \in \mathcal{I}}$. Steps 2.1 through 2.3 of Procedure 1 represent round $k$ of the procedure.

\footnotetext{
${ }^{2}$ Note that $S_{-i}^{\varepsilon}\left(B_{-i}^{0}\right)=\prod_{j \neq i} S_{j}^{\varepsilon}\left(B_{j}^{0}\right)$
} 


\section{Procedure 3.1 Iterated Elimination of F-Dominated Conditional Beliefs}

Initial Step: Define $\Gamma_{i}^{0}(\emptyset)=\left(S_{i}, \Delta\left(S_{-i}\right)\right)$ for each player $i$ and let

1.1) $B_{i}^{1}(\emptyset)=\left\{b_{i}(\emptyset) \in \Delta\left(S_{-i}\right) \mid b_{i}(\emptyset)\left(S_{-i}^{\varepsilon}\left(B_{-i}^{0}\right)\right) \geq F_{i}(\varepsilon)\right.$ for all $\left.\varepsilon \geq 0\right\}$

1.2) $B_{i}^{1}(h)$ is the Bayesian updating of $B_{i}^{1}(\emptyset)$ for every $h$ for which there exists $b_{i}(\emptyset) \in$ $B_{i}^{1}(\emptyset)$ with $b_{i}(\emptyset)\left(S_{-i}(h)\right)>0$

1.3) For $h \in \mathcal{H}$ with $b_{i}(\emptyset)\left(S_{-i}(h)\right)=0$ for all $b_{i}(\emptyset) \in B_{i}^{1}(\emptyset), B_{i}^{1}(h)=\Delta\left(S_{-i}(h)\right)$

Inductive Step: Assume $B_{i}^{k-1}$ is defined for both players i. Define $\Gamma_{i}^{k-1}(\emptyset)=\left(S_{i}, B_{i}^{k-1}(\emptyset)\right)$ for each player $i$, then

2.1) $B_{i}^{k}(\emptyset)=\left\{b_{i}(\emptyset) \in B_{i}^{k-1}(\emptyset) \mid b_{i}(\emptyset)\left(S_{-i}^{\varepsilon}\left(B_{-i}^{k-1}\right)\right) \geq F_{i}(\varepsilon)\right.$ for all $\left.\varepsilon \geq 0\right\}$

2.2) $B_{i}^{k}(h)$ is the Bayesian updating of $B_{i}^{k}(\emptyset)$ for every $h$ for which there exists $b_{i}(\emptyset) \in$ $B_{i}^{k}(\emptyset)$ with $b_{i}(\emptyset)\left(S_{-i}(h)\right)>0$

2.3) For $h \in \mathcal{H}$ with $b_{i}(\emptyset)\left(S_{-i}(h)\right)=0$ for all $b_{i}(\emptyset) \in B_{i}^{k}(\emptyset), B_{i}^{k}(h)=B_{i}^{k-1}(h)$

It is worth noting that Procedure 3.1 is a generalization of the Iterative Conditional Dominance procedure of Shimoji and Watson (1998). More precisely, if $F_{i}(\varepsilon)=1$ for all $\varepsilon \geq 0$ for every $i$, then Iterated Elimination of $F$-Dominated Conditional Beliefs reduces to Iterative Conditional Dominance. Furthermore, theorem 3.2 shows Procedure 3.1 characterizes exactly those first order conditional beliefs that can be held by types expressing common strong belief in $F$-rationality.

Theorem 3.2 The set of first order beliefs $\left(b_{i}^{1}\left(t_{i}, h\right)\right)_{h \in \mathcal{H}_{i}^{*}}$ that can be held by an epistemic type $t_{i}$ expressing up to $k$-fold strong belief in $F$-rationality in some sufficiently rich epistemic model, is exactly the set $\left(B_{i}^{k}(h)\right)_{h \in \mathcal{H}_{i}^{*}}$ of beliefs surviving $k$ rounds of the procedure of Iterated Elimination of $F$-Dominated Conditional Beliefs, for every $k \geq 0$.

\subsubsection{Practical Matters of Implementation}

Notably, the procedure in its current formulation may seem difficult to apply to an actual game, especially if one is aiming to do so without the use of a computer. One way to simplify the implementation of the procedure is through defining, what we call, "the critical $\varepsilon$ of strategy $s_{i}{ }^{\prime}$. From Definition 3.7 it is clear that $\varepsilon$-optimality of any strategy $s_{i}$ for some belief $b_{i}$ requires its expected utility at every $h \in \mathcal{H}_{i}^{*}\left(s_{i}\right)$ to be within $\varepsilon$ of that of the best response to $b_{i}(h)$. The critical $\varepsilon$ of strategy $s_{i}$ is the minimum value of $\varepsilon$ for which there exists a belief $\left(b_{i}(h)\right)_{h \in \mathcal{H}_{i}^{*}\left(s_{i}\right)}$ 
making $s_{i} \varepsilon$-optimal at every $h \in \mathcal{H}_{i}^{*}\left(s_{i}\right)$. Let $\varepsilon^{s_{i}}(k, h)$ be the critical $\varepsilon$ of strategy $s_{i}$ within the decision problem $\Gamma_{i}^{k}(h)$, i.e. the critical $\varepsilon$ of strategy $s_{i}$ at information set $h$ after $k$ rounds of the procedure. Definition 3.8 formally introduces $\varepsilon^{s_{i}}(k, h)$.

Definition 3.8 The critical $\varepsilon$ of strategy $s_{i}$ in decision problem $\Gamma_{i}^{k}(h)$, denoted by $\varepsilon^{s_{i}}(k, h)$, is defined as

$\varepsilon^{s_{i}}(k, h)=\operatorname{Min}\left\{\varepsilon \mid \varepsilon \geq 0, \exists b_{i}(h) \in B_{i}^{k}(h)\right.$ with $\left.u_{i}\left(s_{i}, b_{i}(h)\right) \geq u_{i}\left(s_{i}^{\prime}, b_{i}(h)\right)-\varepsilon \quad \forall s_{i}^{\prime} \in S_{i}(h)\right\}$

To devise a simpler method for the characterization of $\varepsilon^{s_{i}}(k, h)$, certain characteristics of sets $B_{i}^{k}(h)$ need to be established. Lemma 3.1 states the characteristic of $B_{i}^{k}(h)$ that is of most interest to us.

Lemma 3.1 Take some $k \geq 0$ and some player $i \in \mathcal{I}$, then the set $B_{i}^{k}(h)$ is the convex hull of finitely many points, for every $h \in \mathcal{H}_{i}^{*}$.

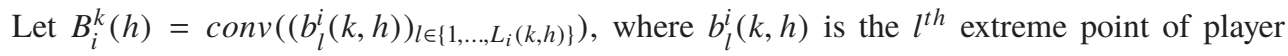
$i$ 's belief set $B_{i}^{k}(h)$ at information set $h$ after $k$ rounds of the recursive procedure. Note that $L_{i}(k, h)<\infty$ for all $h \in \mathcal{H}_{i}^{*}, k \geq 0$ and $i \in \mathcal{I}$. Define the set of extreme points of set $B_{i}^{k}(h)$ as $E B_{i}^{k}(h)=\left\{b_{1}^{i}(k, h), \ldots, b_{L_{i}(k, h)}^{i}(k, h)\right\}$. Decision problem $\Gamma_{i}^{k}(h)$ can be equivalently defined as $\Gamma_{i}^{k}(h)=\left(S_{i}(h), E B_{i}^{k}(h)\right)$. Lemma 3.2 provides a characterization of $\varepsilon^{s_{i}}(k, h)$ that is equivalent to Definition 3.8. For any $s_{i}$ and any $h$, the critical value $\varepsilon^{s_{i}}(k, h)$ is the maximum $\varepsilon$ such that there exists a randomization $r_{i} \in \Delta\left(S_{i}(h)\right)$ that strictly dominates $s_{i}$ within the decision problem $\Gamma_{i}^{k}(h)$ by more than $\varepsilon$, where $r_{i} \in \Delta\left(S_{i}(h)\right)$ is a probability distribution over the set of player $i$ 's strategies leading to information set $h$ other than $s_{i}$.

Lemma 3.2 The critical $\varepsilon^{s_{i}}(k, h)$ of strategy $s_{i}$ in decision problem $\Gamma_{i}^{k}(h)$ is equal to $\varepsilon^{s_{i}}(k, h)=$ $\operatorname{Max}\left\{\varepsilon \mid \varepsilon \geq 0, \exists r_{i} \in \triangle\left(S_{i}(h)\right)\right.$ with $\left.u_{i}\left(r_{i}, b_{l}^{i}(k, h)\right) \geq u_{i}\left(s_{i}, b_{l}^{i}(k, h)\right)+\varepsilon \quad \forall b_{l}^{i}(k, h) \in E B_{i}^{k}(h)\right\}$.

Note that this lemma is a generalization of the classical result implying that any one choice in a static game is optimal for some belief about opponents' choice combinations, if and only if it is not strictly dominated by any other choice or randomization among choices (Pearce, 1984). Moreover, it follows directly from Theorem 1 in MPT (2013) along with Lemma 3.1 above. The critical $\varepsilon$ of strategy $s_{i}$ after round $k$ of the recursive procedure, or $\varepsilon^{s_{i}}(k)$, is formalized in Definition 3.9. 
Definition 3.9 The critical epsilon $\varepsilon^{s_{i}}(k)$ of strategy $s_{i}$ is

$$
\varepsilon^{s_{i}}(k)=\operatorname{Max}\left\{\varepsilon^{s_{i}}(k, h) \mid h \in \mathcal{H}_{i}^{*}\left(s_{i}\right)\right\} .
$$

Consequently, set $S_{i}^{\varepsilon}\left(B_{i}^{k}\right)$, introduced in Definition 3.7, can be redefined in terms of $\varepsilon^{s_{i}}(k)$ as in Corollary

Corollary 3.1 The set of strategies $S_{i}^{\varepsilon}\left(B_{i}^{k}\right)$ of player i that are $\varepsilon$-optimal for some belief $b_{i} \in B_{i}^{k}$ can be defined as $S_{i}^{\varepsilon}\left(B_{i}^{k}\right)=\left\{s_{i} \in S_{i} \mid \varepsilon^{s_{i}}(k) \leq \varepsilon\right\}$.

\subsubsection{An Example: The Centipede Game}

This section presents an example demonstrating how the notion of the critical $\varepsilon$ can facilitate the implementation of the procedure. Consider the Centipede game in Figure 1 and let

$$
F_{i}(\varepsilon)=\left\{\begin{array}{rrr}
\frac{\varepsilon}{3.2} & \text { if } & \varepsilon \leq 3.2 \\
1 & \text { if } & \varepsilon \geq 3.2
\end{array}\right.
$$

The decision problem $\Gamma^{0}(h)$ each player faces at each information set $h$ in round 1 of the procedure will be as follows;

\begin{tabular}{c|ccc}
$\Gamma_{1}^{0}(\emptyset)$ & $\mathrm{T}$ & $\mathrm{PT}$ & $\mathrm{PP}$ \\
\hline $\mathrm{T}$ & 0.4 & 0.4 & 0.4 \\
$\mathrm{PT}$ & 0.2 & 1.6 & 1.6 \\
$\mathrm{PP}$ & 0.2 & 0.8 & 6.4
\end{tabular}

\begin{tabular}{c|cc}
$\Gamma_{1}^{0}\left(h_{1}\right)$ & $\mathrm{PT}$ & $\mathrm{PP}$ \\
\hline $\mathrm{PT}$ & 1.6 & 1.6 \\
$\mathrm{PP}$ & 0.8 & 6.4
\end{tabular}

"Table 2: Decision Problems of Player 1 at the Beginning of the procedure"

\begin{tabular}{c|ccc}
$\Gamma_{2}^{0}(\emptyset)$ & $\mathrm{T}$ & $\mathrm{PT}$ & $\mathrm{PP}$ \\
\hline $\mathrm{T}$ & 0.1 & 0.1 & 0.1 \\
$\mathrm{PT}$ & 0.8 & 0.4 & 0.4 \\
$\mathrm{PP}$ & 0.8 & 3.2 & 1.6
\end{tabular}

\begin{tabular}{c|ccc}
$\Gamma_{2}^{0}\left(h_{2}\right)$ & $\mathrm{T}$ & $\mathrm{PT}$ & $\mathrm{PP}$ \\
\hline $\mathrm{PT}$ & 0.8 & 0.4 & 0.4 \\
$\mathrm{PP}$ & 0.8 & 3.2 & 1.6
\end{tabular}

\begin{tabular}{c|cc}
$\Gamma_{2}^{0}\left(h_{2}^{\prime}\right)$ & $\mathrm{PT}$ & $\mathrm{PP}$ \\
\hline $\mathrm{PP}$ & 3.2 & 1.6
\end{tabular}

"Table 3: Decision Problems of Player 2 at the Beginning of the procedure" 
Strategy $P P$ of player 2 is strictly dominated by 0.23 at information set $h_{2}$ and by 1.6 at information set $h_{2}^{\prime}$, i.e. $\varepsilon_{2}^{P P}\left(1, h_{2}\right)=0.23$ and $\varepsilon_{2}^{P P}\left(1, h_{2}^{\prime}\right)=1.6$. Therefore, the critical $\varepsilon_{2}^{P P}(1)=1.6$. In other words, for strategy $P P$ to be $\varepsilon$-rational for some belief, player 2 has to be willing to tolerate a margin of error of 1.6 or more. Given the form of function $F_{i}$, strategy $P P$ of player 2 cannot at the beginning of the game receive probability more than 0.5 , if player 1 strongly believes in player 2's $F$-rationality. The feasible belief set of player 1 at the beginning of the game $\emptyset$ is shown in the upper left panel of Figure 2. As for player 1, there are no strictly dominated strategies at any $h \in \mathcal{H}_{1}^{*}$, so $S_{1}^{0}\left(B_{1}^{0}\right)=S_{1}$. Thus player 2 can at $\emptyset$ hold any belief about player 1's strategies while believing in the latter's $F$-rationality (upper right panel in Figure 2). Belief sets $B_{1}^{1}\left(h_{1}\right)$ and $B_{2}^{1}\left(h_{2}\right)$ are the Bayesian updating of $B_{1}^{1}(\emptyset)$ and $B_{2}^{1}(\emptyset)$, respectively (the lower panels of Figure 2). In Figure 2, for $B_{i}^{1}(\emptyset)$ the gray filled area is the feasible belief set at $\emptyset$ after round 1 of the procedure, and for $B_{i}^{1}(h)$ with $h \neq \emptyset$ such feasible set is represented by the solid lines. 

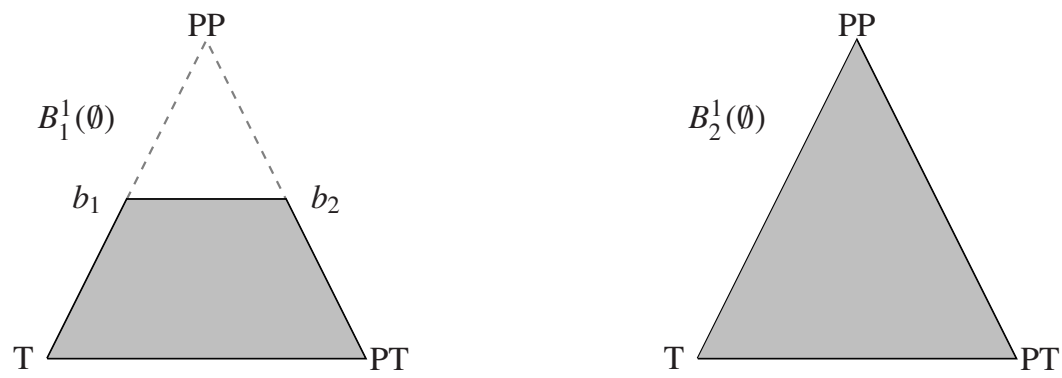

$B_{1}^{1}\left(h_{1}\right)$

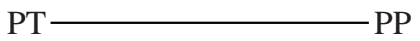

$B_{2}^{1}\left(h_{2}\right)$

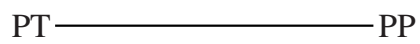

$b_{1}=0.5 T+0.5 P P$
$b_{2}=0.5 P T+0.5 P P$

"Figure 2: Belief Sets of Both Players after Round 1 of the procedure "

Now that the belief set of player 1 is restricted, he would be facing a new restricted decision problem $\Gamma_{1}^{1}=\left\{S_{1}, B_{1}^{1}\right\}$, or equivalently $\Gamma_{1}^{1}=\left\{S_{1},\left(E B_{1}^{1}(h)\right)_{h \in \mathcal{H}_{i}^{*}}\right\}$, where $E B_{1}^{1}(\emptyset)=\left\{T, P T, b_{1}, b_{2}\right\}$ and $E B_{1}^{1}\left(h_{1}\right)=\{P T, P P\}$, as shown in Table 2 .

\begin{tabular}{c|cccc}
\hline$\Gamma_{1}^{1}(\emptyset)$ & $\mathrm{T}$ & $\mathrm{PT}$ & $b_{1}$ & $b_{2}$ \\
\hline $\mathrm{T}$ & 0.4 & 0.4 & 0.4 & 0.4 \\
$\mathrm{PT}$ & 0.2 & 1.6 & 0.9 & 1.6 \\
$\mathrm{PP}$ & 0.2 & 0.8 & 3.3 & 3.6 \\
\hline
\end{tabular}

\begin{tabular}{c|cc}
\hline$\Gamma_{1}^{1}\left(h_{1}\right)$ & $\mathrm{PT}$ & $\mathrm{PP}$ \\
\hline $\mathrm{PT}$ & 1.6 & 1.6 \\
$\mathrm{PP}$ & 0.8 & 6.4 \\
\hline
\end{tabular}

"Table 4: Decision Problems of Player 1 after Round 1"

In his new decision problem, player 1 still has no strictly dominated strategies. The belief set of player 2 therefore remains unrestricted. Moreover, since the belief set of player 2 stayed unrestricted after round 1, player 2's decision problem after round 1 is still the original game. The latter implies no further restrictions on player 1's belief set after round 1. The procedure stops and players 1 and 2's belief sets are as shown in Figure 2 above. 


\subsection{Discussion and Conclusion}

In the Centipede game examined above, it can be shown that CSBR implies that the only reasonable strategy would be for each player to choose "Take" at the first possible chance, i.e. play $T$. This follows from the fact that in games of perfect information, the only reasonable outcome under CSBR is that of backward induction (Battigalli (1997); Battigalli and Siniscalchi (2002)). On the other hand, as shown in the example of section 3.3.2, under CSBFR any node of the game can be reached with positive probability according to players' belief sets under the appropriate functions $\left(F_{i}\right)_{i \in I}$. Consequently, every outcome of the game becomes feasible. This is, however; not a general case for any $\left(F_{i}\right)_{i \in I}$. The above example has shown that this is indeed the case when function $F_{i}$ is the cumulative probability distribution generated by the uniform distribution $U(0,3.2)$ for both players.

The Centipede game has received significant attention due to the discrepancy between theoretical predictions of its outcome and actually observed experimental data. We therefore, consider in this section some more results of this game. We focus on one of the three versions of the Centipede game used in McKelvey and Palfery (1992); namely the one shown in Figure 1. This version of the game is characterized by exponentially increasing payoffs ${ }^{3}$. Assume $F_{i}$ is as follows

$$
F_{i}(\varepsilon)=\left\{\begin{array}{cccc}
\frac{\varepsilon}{x} & & \text { if } & \varepsilon \leq x \\
1 & \text { if } & \varepsilon \geq x
\end{array}\right.
$$

the cumulative probability distribution generated by the uniform distribution $U(0, x)$ for both players. For each information set $h \in \mathcal{H}$ we characterize the minimum $x$ such that information set $h$ can be reached with positive probability at the beginning of the game.

For a value of $x \leq 1.6$, strategy $P P$ of player 2 can never receive positive probability in a belief of player 1 under common strong belief in $F$-rationality. Under $x \leq 0.8$, beliefs of player 2 assigning positive probability to strategy $P P$ of player 1 are all eliminated. Information set $h_{2}^{\prime}$ of player 2 is, therefore, never reached with positive probability at the beginning of the game. In other words, $b_{2}\left(t_{2}, \emptyset\right)\left(P P, t_{1}\right)>0$ can never be the case for any $t_{2}$ expressing common strong belief in $F$-rationality. Moreover, strategy $P T$ of player 2 would receive a probability of zero, if $x \leq 0.4$. In this case no positive probability can be assigned to player 2 passing, i.e. playing either $P P$ or $P T$, and so information set $h_{1}$ of player 1 becomes unreachable.

\footnotetext{
${ }^{3}$ Similar results can be obtained for the linear payoff case, but these are not included here
} 
Finally, if $x \leq 0.2$ the game can only be expected to result in the backward induction outcome, as no information set beyond the beginning of the game $\emptyset$ can be reached with positive probability. Table 4 summarizes these cases ${ }^{4}$, along with the final belief sets $\left(B_{1}^{\infty}(h)\right)_{h \in \mathcal{H}_{1}^{*}}$ and $\left(B_{2}^{\infty}(h)\right)_{h \in \mathcal{H}_{2}^{*}}$ surviving the recursive procedure for both players. Note that these belief sets are shown for specific values of $x$, namely, 1.6, 0.8, 0.4 and less than or equal 0.2 . 


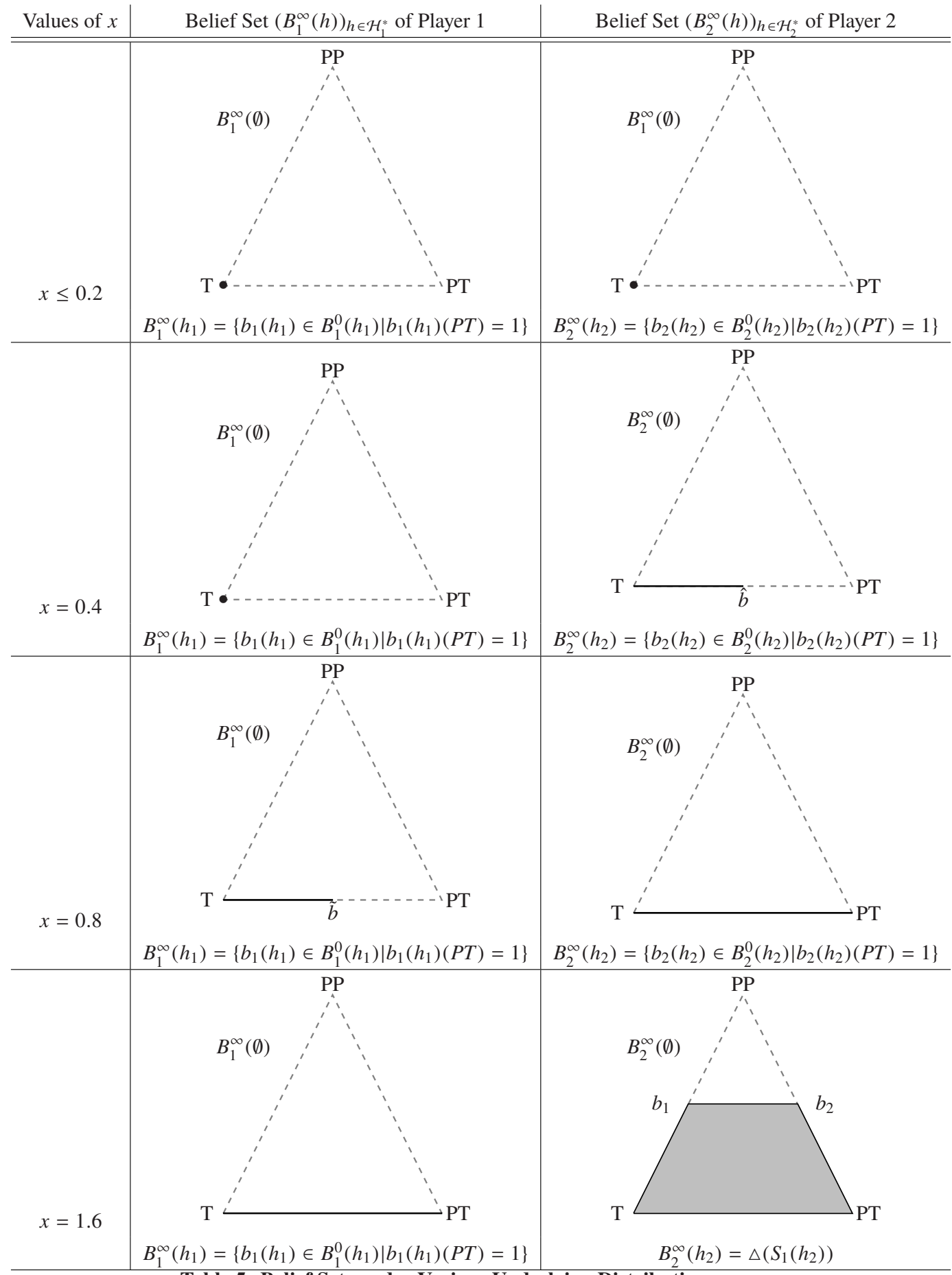

Table 5: Belief Sets under Various Underlying Distributions 
In addition to the above theoretical results, we also consider some experimental results from McKelvey and Palfery (1992). In their paper, McKelvey and Palfery (M\&P) consider three versions of the centipede game, the first of which was reproduced in Figure 1. For the four stage Centipede reported in Figure 1, M\&P employed a total of 58 subjects in three sessions. Sessions 1 and 3 consisted of 20 subjects each while session 2 was run with 18 subjects. In each session participants are divided into two groups, first players and second players. Each member of the first players group is then paired with each of the second players group. Each player in sessions 1 and 3 thus played a total of 10 games, while each subject in session 2 played only 9 games. For every game M\&P reported the node in which either of the two players exited the game by choosing to take.

We consider M\&P data for the first, fifth and ninth game aggregated over the three sessions. For each node $h$, the third column of Table 5, for example, presents the proportion of first games played in all three sessions ending at node $h$. Based on the observed exit node, one can assign a combination of strategies that must have been played by the two players to reach the reported outcome. Such strategy combination we call an observed strategy combination (column two in Table 5). For example, if the game ended at node $h_{2}^{\prime}$ by player 2 choosing to take, this would imply player 1 must have played $P P$, while player 2 must have played $P T$. For other nodes, the observed strategy may be less clear. Take, for example, a game stopping at node $h_{2}$, it is clear player 2 played $T$. But for player 1 all that could be concluded is that he passed at $\emptyset$, i.e. he played either $P T$ or $P P$. In such case we denote player 1's strategy by $P, \cdot$.

\begin{tabular}{c|c|ccc}
\hline Node of Exit & Observed $\left(s_{1}, s_{2}\right)$ & First Game & Fifth Game & Ninth Game \\
\hline$\emptyset$ & $(T,--)$ & 0 & 0.1 & 0.1 \\
$h_{2}$ & $(P \cdot, T)$ & 0.3 & 0.4 & 0.5 \\
$h_{1}$ & $(P T, P \cdot)$ & 0.4 & 0.3 & 0.3 \\
$h_{2}^{\prime}$ & $(P P, P T)$ & 0.2 & 0.2 & 0.1 \\
Pass All & $(P P, P P)$ & 0.1 & 0.1 & 0 \\
\hline
\end{tabular}

Table $^{5} 6$

From Table 6, the low proportion of games ending at node $\emptyset$ becomes evident. The proportion of games displaying the backward induction outcome is in the first game as low as zero, and goes up to a 10 percent in games 5 and 9 . The pair $(P P, P P)$ also occurred only 10 percent of the time in games 1 and 5 and then disappeared by game 9 . The strategy pair $(P P, P T)$ also exhibited a similar pattern. Thus the share of strategy pairs in which player 1 played $P P$ has declined between game 1 and game 9. These previous developments suggest the presence of a learning effect. As players play the game more often, the outcome moves closer to the backward

${ }^{5}$ Source: Author's calculations based on experimental data of McKelvey and Palfery 1992 
induction outcome. However, given the above figures, one has to wonder about the extent of such convergence. This is, however, beyond the topic of our paper.

Results obtained from the M\&P experiment can serve as a basis for some reasonable subjective beliefs. Plugging the probability distribution over each player $i$ 's strategies obtained from Table 5 above into the respective $B_{i}(\emptyset)$, yields Figure 3 below.
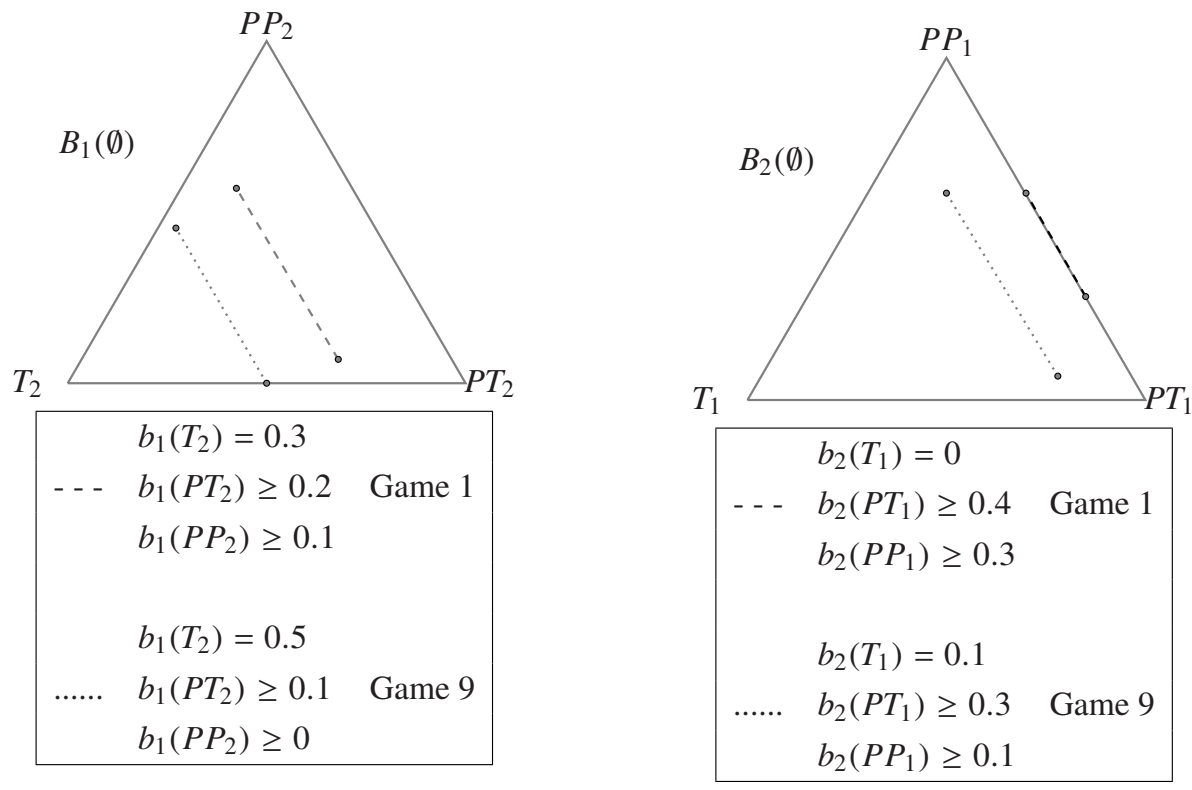

Figure 3: Conditional Belief Sets Based on the M\&P Experiment

Based on the observed frequencies, player 1 in the above Centipede game can legitemately assign some positive probability to player 2 playing pass at node $h_{2}$. This is supported by the two sets of beliefs obtained from games 1 and 9 . The line corresponding to game 1 , for example, in $B_{1}(\emptyset)$ shows a set of beliefs in which probability 0.3 is assigned to player 2 playing $T$ and probability 0.7 to player 2 playing " $P T$ or $P P$ ". Similarily, for the beliefs of player 2 . From $\mathrm{M} \& \mathrm{P}$ data, player 2 can assign, based on game 1 , up to probability 1 to player 1 playing "PT or $P P^{\prime \prime}$ (as represented by the dashed line coinciding with the side of the triangle connecting $P P_{1}$ and $\left.P T_{1}\right)$.

Estimating exact beliefs about opponent's strategies with precision in such a case would be hard to do. The above beliefs are a mere example of the sort of beliefs one could form, if one were to rely on observations of past course of play in the game of interest. Moreover, beliefs at later information sets are hard to estimate based on available data. Moreover, if the above results 
could be viewed as a legitimate basis for a belief held by either player at the beginning of the game, beliefs at later stages would undoubtedly also depend on opponent's behaviour observed by each player. It is worth emphasizing that our analysis of Mckelvy and Palfery's experimental data is far from formal. Instead, we rely on basic descriptive analysis, to draw the reader's attention to the fact that experimental data might well be supporting our model. The extent to which this is the case, if any, is a question for further research. 


\subsection{Appendix}

\section{Proof of Theorem 3.1:}

Let $F T_{i}^{k}$ be the set of types expressing up to $k$-fold strong belief in $F$-rationality, for a function of the form $F_{i}(\varepsilon)=1$ for all $\varepsilon \geq 0$ (Definition 3.4 in section 3.2.3). Moreover, let $S T_{i}^{k}$ be the set of types expressing up to $k$-fold strong belief in rationality (Definition 3.6 in section 3.2.3). By induction on $k$, the following two components of Theorem 3.1 are proved:

(A) $F T_{i}^{k} \subseteq S T_{i}^{k}$

(B) $S T_{i}^{k} \subseteq F T_{i}^{k}$

Induction Assumption: Take $k=0$ then by definition of types $T_{i}=F T_{i}^{0}=S T_{i}^{0}$. The induction assumption therefore becomes

$$
\text { Type } t_{i} \in F T_{i}^{k-1} \text { if and only if } t_{i} \in S T_{i}^{k-1} \text { for any } k \geq 0
$$

Induction Step: Part (A) $[k \geq 1]$ : This requires showing that any $t_{i} \in F T_{i}^{k}$ satisfies the conditions laid out in Definition 3.6 of section 3.2.3, i.e. at every $h \in \mathcal{H}_{i}^{*}$ and whenever the set $\left(S_{-i}(h) \times S T_{-i}^{k-1}\right)^{0} \neq \emptyset$, it must be that

$$
b_{i}\left(t_{i}, h\right)\left(S_{-i}(h) \times S T_{-i}^{k-1}\right)^{0}=1
$$

where $\left(S_{-i}(h) \times S T_{-i}^{k-1}\right)^{\varepsilon}=\left\{\left(s_{-i}, t_{-i}\right) \in\left(S_{-i} \times T_{-i}\right)^{\varepsilon} \mid s_{-i} \in S_{-i}(h)\right.$ and $\left.t_{-i} \in S T_{-i}^{k-1}\right\}$.

From Definition 3.4, a type $t_{i} \in F T_{i}^{k}$ satisfies:

(F.1) Belief $b_{i}\left(t_{i}, \emptyset\right)$ satisfies:

(a) $b_{i}\left(t_{i}, \emptyset\right)\left(S_{-i} \times T_{-i}\right)^{\varepsilon} \geq F_{i}(\varepsilon)$ for all $\varepsilon \geq 0$, and

(b) $b_{i}\left(t_{i}, \emptyset\right)\left(S_{-i} \times F T_{-i}^{k-1}\right)=1$.

(F.2) For every $h \neq \emptyset$,

(a) $b_{i}\left(t_{i}, h\right)$ is the Bayesian updating of some $b_{i}\left(t_{i}^{\prime}, \emptyset\right)$ satisfying condition F.1.(a) with $b_{i}\left(t_{i}^{\prime}, \emptyset\right)\left(S_{-i}(h) \times T_{-i}\right)>0$ whenever possible,

(b) $b_{i}\left(t_{i}, h\right)\left(S_{-i}(h) \times T_{-i}^{k-1}\right)=1$ whenever possible. If at some $h \neq \emptyset$ this is not possible, $b_{i}\left(t_{i}, h\right)\left(S_{-i}(h) \times T_{-i}^{l}\right)=1$ should hold for the highest possible $l<k-1$. 
Since $F_{i}(\varepsilon)=1$ for all $\varepsilon \geq 0$ condition F. 1 becomes $b_{i}\left(t_{i}, \emptyset\right)\left(S_{-i} \times F T_{-i}^{k-1}\right)^{\varepsilon}=1$ for all $\varepsilon \geq 0$ or

$$
b_{i}\left(t_{i}, \emptyset\right)\left(S_{-i} \times F T_{-i}^{k-1}\right)^{0}=1
$$

Equivalently, this means type $t_{i} \in F T_{i}^{k}$ assigns positive probability at the beginning of the game $\emptyset$ only to $\left(s_{-i}, t_{-i}\right)$ where for every $j \neq i s_{j}$ is optimal for $t_{j} \in F T_{j}^{k-1}$. In other words, $\left(s_{-i}, t_{-i}\right)$ receiving positive probability by $t_{i} \in F T_{i}^{k}$, must be such that $\left(s_{-i}, t_{-i}\right) \in\left(S_{-i} \times F T_{-i}^{k-1}\right)^{0}$. From the induction assumption, $t_{-i} \in F T_{-i}^{k-1}$ if and only if $t_{-i} \in S T_{-i}^{k-1}$. Therefore, any $t_{i} \in F T_{i}^{k}$ assigns positive probability at $\emptyset$ only to $\left(s_{-i}, t_{-i}\right) \in\left(S_{-i} \times S T_{-i}^{k-1}\right)^{0}$.

Moreover, condition F.2.(a) means that for any information sets $h \in \mathcal{H}_{i}$

$$
b_{i}\left(t_{i}\right)(h)\left(s_{-i}, t_{-i}\right)=\frac{b_{i}\left(t_{i}^{\prime}, \emptyset\right)\left(s_{-i}, t_{-i}\right)}{b_{i}\left(t_{i}^{\prime}, \emptyset\right)\left(S_{-i}(h) \times T_{-i}\right)}
$$

where $b_{i}\left(t_{i}^{\prime}, \emptyset\right)$ is some belief vector satisfying condition F.1.(a) with $b_{i}\left(t_{i}^{\prime}, \emptyset\right)\left(S_{-i}(h) \times T_{-i}\right)>0$ whenever possible.

Consider the case where such $t_{i}^{\prime}$ exists. Since $b_{i}\left(t_{i}^{\prime}, \emptyset\right)$ satisfies condition F.1.(a), then for $\left(s_{-i}, t_{-i}\right)$ to receive positive probability at $\emptyset$ it must be that $\left(s_{-i}, t_{-i}\right) \in\left(S_{-i} \times F T_{-i}\right)^{0}$. Since $b_{i}\left(t_{i}, h\right)$ is the Bayesian updating of $b_{i}\left(t_{i}^{\prime}, \emptyset\right)$ and since $t_{i}$ satisfies condition F.2.(b), it follows that any $\left(s_{-i}, t_{-i}\right)$ receiving positive probability at $h$ must be in $\left(S_{-i}(h) \times F T_{-i}^{k-1}\right)^{0}$, or by the induction assumption in $\left(S_{-i}(h) \times S T_{-i}^{k-1}\right)^{0}$. Thus any type $t_{i} \in F T_{i}^{k}$ assigns at any $h \in \mathcal{H}_{i}^{*}$ positive probability only to opponents' $\left(s_{-i}, t_{-i}\right)$ where $s_{-i} \in S_{-i}(h)$, for every $j \neq i$ strategy $s_{j}$ is optimal for $t_{j}$ and $t_{-i} \in S T_{-i}^{k-1}$. Thus any $t_{i} \in F T_{i}^{k}$ is also in $S T_{i}^{k}$.

If no such $t_{i}^{\prime}$ exists, then no further restrictions are imposed at $h$ beyond requiring $b_{i}\left(t_{i}, h\right)\left(S_{-i}(h) \times\right.$ $\left.F T_{-i}^{l}\right)=1$ to be satisfied for the highest possible $l<k-1$. Note that since $t_{i} \in F T_{i} \subseteq F T_{i}^{k-1}$, then $t_{i} \in S T_{i}^{k-1}$ by the induction assumption. Furthermore, the induction assumption implies that at $h$, if $\left(S_{-i}(h) \times F T_{-i}^{k-1}\right)=\emptyset$ then $\left(S_{-i}(h) \times S T_{-i}^{k-1}\right)=\emptyset$. Iterating on $k$, it follows from the induction assumption that if $\left(S_{-i}(h) \times F T_{-i}^{l}\right)=\emptyset$ then $\left(S_{-i}(h) \times S T_{-i}^{l}\right)=\emptyset$ for every $l<k-1$. Thus at such $h, t_{i} \in F T_{i}^{k}$ maintains $b_{i}\left(t_{i}, h\right)\left(S_{-i}(h) \times F T_{-i}^{l}\right)=1$ or $b_{i}\left(t_{i}, h\right)\left(S_{-i}(h) \times S T_{-i}^{l}\right)=1$ for the highest possible $l<k-1$. Thus type $t_{i} \in F T_{i}^{k}$ satisfies the conditions that $t_{i} \in S T_{i}^{k}$ needs to satisfy at such $h$. Hence, $F T_{i}^{k} \subseteq S T_{i}^{k}$

$\boldsymbol{P a r t}(\boldsymbol{B})[k \geq 1]$ : Consider some $t_{i} \in S T_{i}^{k}$. Proving part (B) requires showing that such $t_{i}$ satisfies conditions F.1 and F.2. Since $t_{i} \in S T_{i}^{k}$, it follows that at every $h \in \mathcal{H}_{i}^{*}$ whenever there exists some $\left(s_{-i}, t_{-i}\right) \in\left(S_{-i}(h) \times S T_{-i}^{k-1}\right)^{0}, t_{i}$ must assign positive probability only to such $\left(s_{-i}, t_{-i}\right)$. 
From the induction assumption, type $t_{i} \in S T_{i}^{k-1}$ if and only if $t_{i} \in F T_{i}^{k-1}$. Thus a type $t_{i} \in S T_{i}^{k}$ is required to assign at every $h$ positive probability only to $\left(s_{-i}, t_{-i}\right) \in\left(S_{-i}(h) \times F T_{-i}^{k-1}\right)^{0}$ whenever that is possible. Hence, at $\emptyset$, any type $t_{i} \in S T_{i}^{k}$ is required to satisfy $b_{i}\left(t_{i}, \emptyset\right)\left(S_{-i} \times F T_{-i}^{k-1}\right)^{0}=1$ which is exactly conditions F.1.(a) and F.1.(b) combined.

Consider some $h \neq \emptyset$ for which there exists some $\left(s_{-i}, t_{-i}\right) \in\left(S_{-i}(h) \times S T_{-i}^{k-1}\right)^{0}$, or equivalently as per the induction assumption some $\left(s_{-i}, t_{-i}\right) \in\left(S_{-i}(h) \times F T_{-i}^{k-1}\right)^{0}$. By definition of $t_{i} \in S T_{i}^{k}$, $b_{i}\left(t_{i}, h\right)\left(S_{-i}(h) \times S T_{-i}^{k-1}\right)^{0}=1$ or $b_{i}\left(t_{i}, h\right)\left(S_{-i}(h) \times F T_{-i}^{k-1}\right)^{0}=1$, thereby satisfying condition F.2.(b). It can also be easily shown that $b_{i}\left(t_{i}, h\right)$ is a Bayesian updating of some $b_{i}\left(t_{i}^{\prime}, \emptyset\right)$ satisfying condition F.1.(a) with $b_{i}\left(t_{i}^{\prime}, \emptyset\right)\left(S_{-i}(h) \times T_{-i}\right)>0$. Consider some $t_{i}^{\prime}$ with $b_{i}\left(t_{i}^{\prime}, \emptyset\right)=b_{i}\left(t_{i}, h\right)$. Thus $b_{i}\left(t_{i}^{\prime}, \emptyset\right)\left(S_{-i}(h) \times F T_{-i}^{k-1}\right)=1$, which satisfies the $F$-condition give $F_{i}(\varepsilon)=1$ for all $\varepsilon \geq 0$ and has $b_{i}\left(t_{i}^{\prime}, \emptyset\right)\left(S_{-i}(h) \times T_{-i}\right)>0$. Hence, in this case, type $t_{i} \in S T_{i}^{k}$ satisfies conditions F.2.(a) and (b).

Consider some $h \neq \emptyset$ where $\left(S_{-i}(h) \times S T_{-i}^{k-1}\right)^{0}=\emptyset$. As shown above, whenever $\left(S_{-i}(h) \times S T_{-i}^{l}\right)=$ $\emptyset$ it is also the case that $\left(S_{-i}(h) \times F T_{-i}^{l}\right)=\emptyset$ for every $l<k-1$ as a result of the induction assumption. Type $t_{i}$ satisfying $b_{i}\left(t_{i}, h\right)\left(S_{-i}(h) \times S T_{-i}^{l}\right)^{0}=1$ for the highest possible $l<k-1$ then implies $b_{i}\left(t_{i}, h\right)\left(S_{-i}(h) \times F T_{-i}^{k-1}\right)^{0}=1$ for that same maximum $l$. Since $S T_{i}^{k} \subseteq S T_{i}^{k-1}$, it follows that $t_{i} \in S T_{i}^{k}$ is also $t_{i} \in S T_{i}^{k-1}$, and so $t_{i} \in F T_{i}^{k-1}$ by the induction assumption. Thus type $t_{i} \in S T_{i}^{k}$ is also in $F T_{i}^{k-1}$ and satisfies condition F.2.(b) for the highest possible $l$. It remains to show that $b_{i}\left(t_{i}, h\right)$ is the Bayesian updating of some $b_{i}\left(t_{i}^{\prime}, \emptyset\right)$ satisfying F.1.(a). Note that this can be shown through the same steps applied in the previous paragraph. Thus $t_{i} \in S T_{i}^{k}$ satisfies all the conditions that a $t_{i} \in F T_{i}^{k}$ should satisfy at such an $h$. It is therefore possible to conclude that $S T_{i}^{k} \subseteq F T_{i}^{k}$.

\section{Proof of Theorem 3.2:}

Let $T_{i}^{k}$ be the set of epistemic types expressing up to $k$-fold strong belief in $F$-rationality as in Definitions 3.3 and 3.4. Also let belief sets $\left(B_{i}^{k}(h)\right)_{h \in \mathcal{H}^{*}}$ be as defined in Procedure 3.1. Proving Theorem 3.2 requires proving two parts:

(A) Any type $t_{i} \in T_{i}^{k}$ expressing up to $k$-fold strong belief in $F$-rationality must hold a first order conditional belief vector $b_{i}^{1}\left(t_{i}\right)=\left(b_{i}^{1}\left(t_{i}, h\right)\right)_{h \in \mathcal{H}_{i}^{*}}$ such that $b_{i}^{1}\left(t_{i}\right) \in B_{i}^{k}$, i.e. $b_{i}^{1}\left(t_{i}, h\right) \in B_{i}^{k}(h)$ for all $h \in \mathcal{H}_{i}^{*}$.

(B) Any conditional belief vector $\left(b_{i}(h)\right)_{h \in \mathcal{H}_{i}^{*}}$ with $b_{i}(h) \in B_{i}^{k}(h)$ for all $h \in \mathcal{H}_{i}^{*}$ can be held as a first order conditional belief vector $\left(b_{i}^{1}\left(t_{i}, h\right)\right)_{h \in \mathcal{H}_{i}^{*}}$ by a type $t_{i}$ expressing up to $k$-fold strong belief in $F$-rationality, i.e. for $t_{i} \in T_{i}^{k}$. 
We prove those two directions by induction on $k$. Each part is first shown to hold for $k=0$, thereby providing grounds for the induction assumption. Parts A and B are then subsequently proven for any $k \geq 1$.

Part A: Induction Start: Let $k=0$ and consider some $t_{i} \in T_{i}^{0}$. By definition of $t_{i}, b_{i}^{1}\left(t_{i}, h\right) \in$ $\triangle\left(S_{-i}(h)\right)$ for every $h \in \mathcal{H}_{i}^{*}$. The set of first order belief vectors surviving 0 rounds of the recursive procedure $B_{i}^{0}$ can by definition be written as $B_{i}^{0}(h)=\Delta\left(S_{-i}(h)\right)$ for every $h$. This implies that for any $h \in \mathcal{H}_{i}^{*}$ and for any $t_{i}, b_{i}^{1}\left(t_{i}, h\right) \in B_{i}^{0}(h)$. Thus proving part (A) for $k=0$.

Part A: Induction Assumption: Any type $t_{i} \in T_{i}^{k-1}$ expressing up to $(k-1)$-fold strong belief in $F$-rationality must hold a first order conditional belief vector $b_{i}^{1}\left(t_{i}\right)=\left(b_{i}^{1}\left(t_{i}, h\right)\right)_{h \in \mathcal{H}_{i}^{*}}$ such that $b_{i}^{1}\left(t_{i}\right) \in B_{i}^{k-1}$, i.e. $b_{i}^{1}\left(t_{i}, h\right) \in B_{i}^{k-1}(h)$ for all $h \in \mathcal{H}_{i}^{*}$.

Part A: Induction Step: Let $t_{i} \in T_{i}^{k}$. From Definitions 3.3 and 3.4, $t_{i}$ satisfies the following two conditions:

(T.1) Belief $b_{i}\left(t_{i}, \emptyset\right)$ satisfies the following:

(a) F-condition: $b_{i}\left(t_{i}, \emptyset\right)\left(S_{-i} \times T_{-i}\right)^{\varepsilon} \geq F_{i}(\varepsilon)$ for all $\varepsilon \geq 0$, and

(b) $b_{i}\left(t_{i}, \emptyset\right)\left(S_{-i} \times T_{-i}^{k-1}\right)=1$.

(T.2) For every $h \neq \emptyset$ :

(a) $b_{i}\left(t_{i}, h\right)$ is the Bayesian updating of some $b_{i}\left(t_{i}^{\prime}, \emptyset\right)$ satisfying the $F$ condition with $b_{i}\left(t_{i}^{\prime}, \emptyset\right)\left(S_{-i}(h) \times T_{-i}\right)>0$ whenever possible,

(b) $b_{i}\left(t_{i}, h\right)\left(S_{-i}(h) \times T_{-i}^{k-1}\right)=1$ whenever possible. If at some $h \neq \emptyset$ this is not possible, $b_{i}\left(t_{i}, h\right)\left(S_{-i}(h) \times T_{-i}^{l}\right)=1$ should hold for the highest possible $l<k-1$.

Showing $t_{i} \in T_{i}^{k}$ must have a first order belief vector $b_{i}^{1}\left(t_{i}\right) \in B_{i}^{k}$, requires showing $b_{i}^{1}\left(t_{i}, h\right)$ satisfies the conditions characterizing $B_{i}^{k}(h)$ for every $h$ :

(A.1) $b_{i}^{1}\left(t_{i}, \emptyset\right)\left(S_{-i}^{\varepsilon}\left(B_{-i}^{k-1}\right)\right) \geq F_{i}(\varepsilon)$ for all $\varepsilon \geq 0$

(A.2) $b_{i}^{1}\left(t_{i}, h\right) \in B_{i}^{k}(h)$ for all $h \neq \emptyset$

Recall from Definition 3.2 that $\left(S_{-i} \times T_{-i}\right)^{\varepsilon}:=\left\{\left(s_{-i}, t_{-i}\right) \in\left(S_{-i} \times T_{-i}\right) \mid s_{j}\right.$ is $\varepsilon$-optimal for $t_{j} \forall j \neq$ $i$. Let $R_{-i}^{\varepsilon}=\left(S_{-i} \times T_{-i}\right)^{\varepsilon}$ for every $\varepsilon \geq 0$. Condition T.1 becomes $b_{i}\left(t_{i}, \emptyset\right)\left(R_{-i}\right)^{\varepsilon} \geq F_{i}(\varepsilon)$ for all $\varepsilon \geq 0$ and $b_{i}\left(t_{i}, \emptyset\right)\left(S_{-i} \times T_{-i}^{k-1}\right)=1$. Let $R_{-i}^{\varepsilon, k-1}=\left\{\left(s_{-i}, t_{-i}\right) \in R_{-i}^{\varepsilon} \mid t_{-i} \in T_{-i}^{k-1}\right\}$. Condition T.1.(b) stating that any type $t_{i} \in T_{i}^{k}$ must have $b_{i}\left(t_{i}, \emptyset\right)\left(S_{-i} \times T_{-i}^{k-1}\right)=1$, implies that any $\left(s_{-i}, t_{-i}\right)$ 
receives positive probability by such $t_{i}$ only if $\left(s_{-i}, t_{-i}\right) \in R_{-i}^{\varepsilon, k-1}$ for some $\varepsilon \geq 0$. Condition T.1 then implies

$$
b_{i}\left(t_{i}, \emptyset\right)\left(R_{-i}^{\varepsilon, k-1}\right) \geq F_{i}(\varepsilon) \quad \text { for all } \varepsilon \geq 0
$$

implying,

$$
b_{i}\left(t_{i}, \emptyset\right)\left(\operatorname{Proj}_{S_{-i}} R_{-i}^{\varepsilon, k-1} \times T_{-i}\right) \geq F_{i}(\varepsilon) \quad \text { for all } \varepsilon \geq 0 .
$$

Note that $\operatorname{Proj}_{S_{-i}} R_{-i}^{\varepsilon, k-1}=\left\{s_{-i} \in S_{-i} \mid s_{j}\right.$ is $\varepsilon$-optimal for some $\left.t_{j} \in T_{j}^{k-1} \forall j \neq i\right\}$. The induction assumption implies that any type $t_{j} \in T_{j}^{k-1}$ has a first order conditional belief vector $b_{j}^{1}\left(t_{j}, h\right) \in B_{j}^{k-1}(h)$ for all $h \in \mathcal{H}_{-i}^{*}$. Recall that $S_{-i}^{\varepsilon}\left(B_{-i}^{k-1}\right)=\left\{s_{-i} \in S_{-i} \mid \varepsilon^{s_{j}}(k-1) \leq \varepsilon \forall j \neq i\right\}$, i.e. $S_{-i}^{\varepsilon}\left(B_{-i}^{k-1}\right)$ is the set of strategy profiles of player $i$ 's opponents, where every $s_{j} \in S_{j}^{\varepsilon}\left(B_{j}^{k-1}\right)$ is $\varepsilon$-optimal for some $b_{j} \in B_{j}^{k-1}$. It follows that $\operatorname{Proj}_{S_{-i}} R_{-i}^{\varepsilon, k-1} \subseteq S_{-i}^{\varepsilon}\left(B_{-i}^{k-1}\right)$.

Hence, it follows that for every $\varepsilon \geq 0$

$$
b_{i}^{1}\left(t_{i}, \emptyset\right)\left(S_{-i}^{\varepsilon}\left(B_{-i}^{k-1}\right)\right) \geq b_{i}^{1}\left(t_{i}, \emptyset\right)\left(\operatorname{Proj}_{S_{-i}} R_{-i}^{\varepsilon, k-1}\right) \geq b_{i}\left(t_{i}, \emptyset\right)\left(R_{-i}^{\varepsilon, k-1}\right) \geq F_{i}(\varepsilon) .
$$

This proves that any type $t_{i} \in T_{i}^{k}$ satisfies condition A.1. Now consider an information set $h \neq \emptyset$. From condition T.2.(a), $b_{i}\left(t_{i}, h\right)$ is the Bayesian updating of some $b_{i}\left(t_{i}^{\prime}, \emptyset\right)$ satisfying the $F$-condition with $b_{i}\left(t_{i}^{\prime}, \emptyset\right)\left(S_{-i}(h) \times T_{-i}\right)>0$ whenever possible. Two cases are thus to be considered.

Case (i): There exists such a $b_{i}\left(t_{i}^{\prime}, \emptyset\right)$ for some $t_{i}^{\prime}$ possibly outside the epistemic model, then $\overline{b_{i}\left(t_{i}, h\right)}$ is the Bayesian updating of $b_{i}\left(t_{i}^{\prime}, \emptyset\right)$ satisfying the $F$-condition and $b_{i}\left(t_{i}, h\right)\left(S_{-i}(h) \times\right.$ $\left.T_{-i}^{k-1}\right)=1$. In other words, $b_{i}\left(t_{i}, h\right)$ is the Bayesian updating of a belief of the form

$$
b_{i}\left(t_{i}^{\prime}, \emptyset\right)\left(S_{-i}(h) \times T_{-i}^{k-1}\right)^{\varepsilon} \geq F_{i}(\varepsilon) \quad \forall \varepsilon \geq 0 .
$$

Recall the definition of $R_{-i}^{\varepsilon, k-1}$ defined above. Thus, for every $\varepsilon \geq 0$,

$$
\left(S_{-i}(h) \times T_{-i}^{k-1}\right)^{\varepsilon} \subseteq R_{-i}^{\varepsilon, k-1} .
$$

Consequently,

$$
\operatorname{Proj}_{S_{-i}}\left(S_{-i}(h) \times T_{-i}^{k-1}\right)^{\varepsilon} \subseteq \operatorname{Proj}_{S_{-i}} R_{-i}^{\varepsilon, k-1} .
$$

Moreover, as shown above,

$$
\operatorname{Proj}_{S_{-i}}\left(S_{-i}(h) \times T_{-i}^{k-1}\right)^{\varepsilon} \subseteq \operatorname{Proj}_{S_{-i}} R_{-i}^{\varepsilon, k-1} \subseteq S_{-i}^{\varepsilon}\left(B_{-i}^{k-1}\right) .
$$


Hence, $b_{i}\left(t_{i}, h\right)$ is the Bayesian updating of a belief

$$
b_{i}^{1}\left(t_{i}^{\prime}, \emptyset\right)\left(S_{-i}^{\varepsilon}\left(B_{-i}^{k-1}\right)\right) \geq b_{i}^{1}\left(t_{i}^{\prime}, \emptyset\right)\left(\operatorname{Proj}_{S_{-i}}\left(S_{-i}(h) \times T_{-i}^{k-1}\right)^{\varepsilon}\right) \geq F_{i}(\varepsilon) \quad \forall \varepsilon \geq 0 .
$$

Thus $b_{i}\left(t_{i}, h\right)$ is the Bayesian updating of a belief $b_{i}\left(t_{i}^{\prime}, \emptyset\right) \in B_{i}^{k}(\emptyset)$.

Case (ii): Such $b_{i}\left(t_{i}^{\prime}, \emptyset\right)$ does not exist. Since sufficient richness requires the epistemic model to include at least one type $t_{i}^{\prime}$ satisfying the $F$-condition with $b_{i}\left(t_{i}^{\prime}, \emptyset\right)\left(S_{-i}(h) \times T_{-i}^{k-1}\right)>0$ whenever that is possible, assuming there exist no such type implies there is no such type inside or outside the model. Thus no further restrictions can be imposed on $b_{i}\left(t_{i}, h\right)$ beyond it being the Bayesian updating of some $b_{i}\left(t_{i}^{\prime}, \emptyset\right)$ where $b_{i}\left(t_{i}^{\prime}, \emptyset\right)\left(S_{-i}(h) \times T_{-i}^{k-2}\right)>0$ whenever possible. Since $t_{i} \in T_{i}^{k}$ it follows by definition of $T_{i}^{k}$ that $t_{i} \in T_{i}^{k-1}$. By the induction assumption, it then follows that $b_{i}^{1}\left(t_{i}\right) \in B_{i}^{k-1}$, and therefore also $b_{i}^{1}\left(t_{i}, h\right) \in B_{i}^{k-1}(h)$.

We prove by contradiction that in such case there exists no $b_{i}(\emptyset) \in B_{i}^{k}(\emptyset)$ with $b_{i}(\emptyset)\left(S_{-i}(h)\right)>0$. Assume $b_{i}^{*}(\emptyset) \in B_{i}^{k}(\emptyset)$ is such belief. Let type $t_{i}^{*}$ be a type of player $i$ with first order conditional beliefs $b_{i}^{1}\left(t_{i}^{*}, \emptyset\right)=b_{i}^{*}(\emptyset)$ and $b_{i}^{1}\left(t_{i}^{*}, h\right)$ being the Bayesian updating of $b_{i}^{*}(\emptyset)$. Such type $t_{i}^{*}$ would then have to be included in the model by the richness condition, which creates a contradiction. Since the Bayesian updating of $B_{i}^{k}(\emptyset)$ at $h$ is no more possible, it follows from Procedure 3.1 that $B_{i}^{k}(\emptyset)=B_{i}^{k-1}(\emptyset)$. Since $b_{i}^{1}\left(t_{i}, h\right) \in B_{i}^{k-1}(h)$, it follows that $b_{i}^{1}\left(t_{i}, h\right) \in B_{i}^{k}(h)$.

Part B: Construction of Belief and Types : Proving this part requires the construction of an epistemic model. Consider a countable epistemic model $M$ assigning to each player $i$ the set of types $T_{i}$. Model $M$ is constructed in two steps; first beliefs then types of each player.

\section{Belief Construction:}

For every $k$ and every $s_{i} \in S_{i}$, let $b_{i}^{s_{i}, k}=\left(b_{i}^{s_{i}, k}(h)\right)_{h \in \mathcal{H}_{i}^{*}}$ be a first order conditional belief vector such that $s_{i}$ is exactly $\varepsilon^{s_{i}}(k)$-optimal for $b_{i}^{s_{i}, k}$, where $b_{i}^{s_{i}, k}(h) \in B_{i}^{k}(h)$ for every $h \in \mathcal{H}_{i}^{*}$ and every $k \geq 0$. Note that $\varepsilon^{s_{i}}(k)$ is the critical $\varepsilon$ of strategy $s_{i}$ in round $k$ of the recursive procedure as in Definitions 3.8 and 3.9, and lemma 3.2 restated below.

Definition 3.8: The critical $\varepsilon$ of strategy $s_{i}$ in decision problem $\Gamma_{i}^{k}(h)$, denoted by $\varepsilon^{s_{i}}(k, h)$, is defined as

$\varepsilon^{s_{i}}(k, h)=\operatorname{Min}\left\{\varepsilon \mid \varepsilon \geq 0, \exists b_{i}(h) \in B_{i}^{k}(h)\right.$ with $\left.u_{i}\left(s_{i}, b_{i}(h)\right) \geq u_{i}\left(s_{i}^{\prime}, b_{i}(h)\right)-\varepsilon \quad \forall s_{i}^{\prime} \in S_{i}(h)\right\}$ 
Lemma 3.2: The critical $\varepsilon^{s_{i}}(k, h)$ of strategy $s_{i}$ in decision problem $\Gamma_{i}^{k}(h)$ is equal to $\varepsilon^{s_{i}}(k, h)=$ $\operatorname{Max}\left\{\varepsilon \mid \varepsilon \geq 0, \exists r_{i} \in \Delta\left(S_{i}(h)\right)\right.$ with $\left.u_{i}\left(r_{i}, b_{l}^{i}(k, h)\right) \geq u_{i}\left(s_{i}, b_{l}^{i}(k, h)\right)+\varepsilon \quad \forall b_{l}^{i}(k, h) \in E B_{i}^{k}(h)\right\}$

Definition 3.9: The critical epsilon $\varepsilon^{s_{i}}(k)$ of strategy $s_{i}$ is

$$
\varepsilon^{s_{i}}(k)=\operatorname{Max}\left\{\varepsilon^{s_{i}}(k, h) \mid h \in \mathcal{H}_{i}^{*}\left(s_{i}\right)\right\} .
$$

\section{Type Construction:}

The countable set of types $T_{i}$ of player $i$ in model $M$ has two components; $T_{i}^{S}$ and $T_{i}^{A}$, i.e. $T_{i}=T_{i}^{S} \cup T_{i}^{A}$. Define the set

$$
T_{i}^{S}:=\left\{t_{i}^{s_{i}, k} \mid s_{i} \in S_{i} \text { and } k \geq 0\right\}
$$

where every $t_{i}^{s_{i}, k} \in T_{i}^{S}$ is such that

$$
b_{i}\left(t_{i}^{s_{i}, k}, h\right)\left(s_{-i}, t_{-i}\right)= \begin{cases}b_{i}^{s_{i}, k}(h)\left(s_{-i}\right) \forall h \in \mathcal{H}_{i}^{*} & \text { if } t_{-i}=t_{-i}^{s_{-i}, k-1} \\ 0 & \text { Otherwise }\end{cases}
$$

As for the second component of set $T_{i}$, set $T_{i}^{A}$ consists of one type $t_{i}^{b_{i}, k} \in T_{i}^{A}$ for every $k$. Each $t_{i}^{b_{i}, k}$ holds a first order belief $b_{i}^{1}\left(t_{i}^{b_{i}, k}, h\right) \in B_{i}^{k}(h)$ for all $h \in \mathcal{H}_{i}^{*}$. Thus for every $k$ one type holding some arbitrary belief in $B_{i}^{k}$ is constructed. Define the set $T_{i}^{A}=\left\{t_{i}^{b_{i}, k} \mid\right.$ for every $k \geq$ $0, b_{i}$ is one arbitrary belief in $B_{i}^{k}$ \}. Every $t_{i}^{b_{i}, k} \in T_{i}^{A}$ is defined by

$$
b_{i}\left(t_{i}^{b_{i}, k}, h\right)\left(s_{-i}, t_{-i}\right)= \begin{cases}b_{i}(h)\left(s_{-i}\right) \in B_{i}^{k}(h) \forall h \in \mathcal{H}_{i}^{*} & \text { if } t_{-i}=t_{-i}^{s_{-i}, k-1} \\ 0 & \text { Otherwise }\end{cases}
$$

Let $t_{i}^{k}$ denote any type in the set $T_{i}=T_{i}^{S} \cup T_{i}^{A}$. From the definitions above, it follows that for any $t_{i}^{k} \in T_{i}$, the first order belief vector $b_{i}^{1}\left(t_{i}^{k}\right) \in B_{i}^{k}$ for some $k \geq 0$, by construction of both $T_{i}^{S}$ and $T_{i}^{A}$. Any type $t_{i}^{k} \in T_{i}$, therefore, satisfies conditions A.1 and A.2 above which characterize $B_{i}^{k}$. Note that the set $T_{i}$ may include redundant types, as $T_{i}^{S} \cap T_{i}^{A}$ may not be empty. These are then to be omitted from $T_{i}$. Note that while the basic idea of the proof depends on set $T_{i}^{S}$, the set of types $T_{i}^{A}$ is introduced to show that the proof holds for any arbitrary belief. This part is proven by induction on $k$. Just like in part A, we start by considering the case of $k=0$ upon which we base the induction assumption. Then the proof proceeds in two steps. Step 1 shows that any 
type $t_{i}^{k} \in T_{i}$ satisfies condition T.1, while Step 2 shows that such type also satisfies condition T.2.

Part B: Induction Start: Let $\left(b_{i}(h)\right)_{h \in \mathcal{H}_{i}^{*}}$ be an arbitrary conditional belief vector such that $b_{i}(h) \in B_{i}^{0}(h)$ for every $h \in \mathcal{H}_{i}^{*}$. By definition, $B_{i}^{0}(h)=\Delta\left(S_{-i}(h)\right)$ for all $h \in \mathcal{H}_{i}^{*}$, so $b_{i}(h) \in \Delta\left(S_{-i}(h)\right)$ for all $h \in \mathcal{H}_{i}^{*}$. As noted in part (A) above, a type $t_{i} \in T_{i}^{0}$, by definition, has a first order conditional belief that is some $b_{i}^{1}\left(t_{i}, h\right) \in \Delta\left(S_{-i}(h)\right)$ for all $h \in \mathcal{H}_{i}^{*}$. Since $b_{i}^{1}\left(t_{i}, h\right)$ can be any belief in $\Delta\left(S_{-i}(h)\right)$ for every $h$, it follows that the arbitrary conditional belief vector $\left(b_{i}(h)\right)_{h \in \mathcal{H}_{i}^{*}}$ chosen above can be held as a first order conditional belief by some type $t_{i} \in T_{i}^{0}$ in some epistemic model, since $T_{i}^{0}=T_{i}$.

Part B: Induction Assumption: Any conditional belief vector $\left(b_{i}(h)\right)_{h \in \mathcal{H}_{i}^{*}}$ with $b_{i}(h) \in B_{i}^{k-1}(h)$ for all $h \in \mathcal{H}_{i}^{*}$ can be held as a first order conditional belief vector $\left(b_{i}^{1}\left(t_{i}, h\right)\right)_{h \in \mathcal{H}_{i}^{*}}$ by a type $t_{i}$ expressing up to $k-1$-fold strong belief in $F$-rationality, i.e. for $t_{i} \in T_{i}^{k-1}$.

\section{Part B: Induction Step:}

Take some $k \geq 1$ and a strategy profile $s_{-i} \in S_{-i}^{\varepsilon}\left(B_{-i}^{k-1}\right)$ for some $\varepsilon \geq 0$. By definition of $S_{-i}^{\varepsilon}\left(B_{-i}^{k-1}\right)$, it must be that $\varepsilon \geq \varepsilon^{s_{j}}(k-1)$ for every $j \neq i$. Moreover, consider a profile of types $t_{-i}^{s_{-i}, k-1}$, and note that $b_{j}^{1}\left(t_{j}^{s_{j}, k-1}, h\right) \in B_{j}^{k-1}(h)$ for every $h \in \mathcal{H}_{j}^{*}$ and for every $j \neq i$, by construction. Since every $s_{j}$ in the profile $s_{-i}$ is $\varepsilon^{s_{j}}(k-1)$-optimal for $t_{j}^{s_{j}, k-1}$ by definition, the profile of combinations $\left(s_{-i}, t_{-i}^{s_{-i}, k-1}\right) \in R_{-i}^{\varepsilon}$ for every $\varepsilon \geq \max _{j \neq i} \varepsilon^{s_{j}}(k-1)$, where $R_{-i}^{\varepsilon}$ is as defined in part A above. By construction of types, there exists for every profile of strategies $s_{-i} \in S_{-i}^{\varepsilon}\left(B_{-i}^{k-1}\right)$ some profile of types $t_{-i}^{s_{-i}, k-1} \in T_{-i}^{S}$, where $s_{j}$ is $\varepsilon$-optimal for $b_{j}^{1}\left(t_{j}^{s_{j}, k-1}\right)$ for every $j \neq i$. Therefore, any profile of combinations $\left(s_{-i}, t_{-i}^{s_{-i}, k-1}\right) \in R_{-i}^{\varepsilon}$, whenever $s_{-i} \in S_{-i}^{\varepsilon}\left(B_{-i}^{k-1}\right)$.

Now take some type $t_{i}^{k} \in T_{i}$. Since $t_{i}^{k}$ satisfies condition A.1 by definition, it follows that

$$
b_{i}^{1}\left(t_{i}^{k}, \emptyset\right)\left(S_{-i}^{\varepsilon}\left(B_{-i}^{k-1}\right)\right) \geq F_{i}(\varepsilon) \quad \text { for all } \varepsilon \geq 0
$$

Note that $S_{-i}^{\varepsilon}\left(B_{-i}^{k-1}\right)$ contains those strategy combinations $s_{-i} \in S_{-i}$ where for every $j \neq i$ strategy $s_{j}$ has a critical $\varepsilon$ of $\varepsilon^{s_{j}}(k-1) \leq \varepsilon$, i.e. for which there exist $b_{j} \in B_{j}^{k-1}$ making it $\varepsilon^{s_{j}}(k-1)$-rational. By definition of $t_{j}^{s_{j}, k}$, it follows that

$$
b_{i}\left(t_{i}^{k}, \emptyset\right)\left[\bigcup_{s_{-i} \in S_{-i}^{\varepsilon}\left(B_{-i}^{k-1}\right)}\left(s_{-i}, t_{-i}^{s_{-i}, k-1}\right)\right] \geq F_{i}(\varepsilon) \text { for all } \varepsilon \geq 0
$$


Since every such profile of strategy-type combinations $\left(s_{-i}, t_{-i}^{s_{-i}, k-1}\right) \in R_{-i}^{\varepsilon}$, it then follows that for all $\varepsilon \geq 0$

$$
b_{i}\left(t_{i}^{k}, \emptyset\right)\left(R_{-i}^{\varepsilon}\right) \geq b_{i}\left(t_{i}^{k}, \emptyset\right)\left[\bigcup_{s_{-i} \in S_{-i}^{\varepsilon}\left(B_{-i}^{k-1}\right)}\left(s_{-i}, t_{-i}^{s_{-i}, k-1}\right)\right]=b_{i}^{1}\left(t_{i}^{k}, \emptyset\right)\left(S_{-i}^{\varepsilon}\left(B_{-i}^{k-1}\right)\right) \geq F_{i}(\varepsilon)
$$

which implies

$$
b_{i}\left(t_{i}^{k}, \emptyset\right)\left(R_{-i}^{\varepsilon}\right) \geq F_{i}(\varepsilon) \quad \text { for all } \varepsilon \geq 0
$$

This proves that type $t_{i}^{k} \in T_{i}$ satisfies condition T.1.(a). By construction of types, $t_{i}^{k}$ assigns positive probability only to opponents' $\left(s_{-i}, t_{-i}\right)$ where $t_{-i}=t_{-i}^{s_{-i}, k-1}$. Also by definition, $b_{j}^{1}\left(t_{j}^{s_{j}, k-1}, h\right) \in B_{j}^{k-1}(h)$ for all $h \in \mathcal{H}_{j}^{*}$ for every $j \neq i$. It follows from the induction assumption that all such types $t_{j}^{s_{j}, k-1}$, where $j \neq i$, express up to $(k-1)$-fold strong belief in $F$-rationality. This proves condition T.1.(b), i.e.

$$
b_{i}\left(t_{i}^{k}, \emptyset\right)\left(S_{-i} \times T_{-i}^{k-1}\right)=1 .
$$

Thus any types $t_{i}^{k} \in T_{i}$ satisfies condition T.1.

Take some $t_{i}^{k} \in T_{i}$, then by construction $b_{i}^{1}\left(t_{i}^{k}, h\right) \in B_{i}^{k}(h)$ for all $h \neq \emptyset$. By definition, $B_{i}^{k}(h)$ is the Bayesian updating of $B_{i}^{k}(\emptyset)$, for every $h \neq \emptyset$ for which there exists at least some $b_{i}(\emptyset) \in B_{i}^{k}(\emptyset)$ with $b_{i}(\emptyset)\left(S_{-i}(h)\right)>0$. In what follows we show that any such $t_{i}^{k}$ satisfies condition T.2. For that purpose, two cases should be distinguished:

Case (i): There exists such $b_{i}(\emptyset) \in B_{i}^{k}(\emptyset)$ with $b_{i}(\emptyset)\left(S_{-i}(h)\right)>0$, so $B_{i}^{k}(h)$ is the Bayesian updating of $B_{i}^{k}(\emptyset)$. Consider the conditional belief $b_{i}\left(t_{i}^{k}, h\right)$, where type $t_{i}^{k}$ of player $i$ is as defined above and where $b_{i}^{1}\left(t_{i}^{k}, h\right) \in B_{i}^{k}(h)$ by construction. Construct the type $t_{i}^{\prime}$ with $b_{i}\left(t_{i}^{\prime}, \emptyset\right)=b_{i}\left(t_{i}^{k}, h\right)$. It is easy to see that $b_{i}\left(t_{i}^{k}, h\right)$ is the Bayesian updating of such $b_{i}\left(t_{i}^{\prime}, \emptyset\right)$. Furthermore, note that $b_{i}\left(t_{i}^{\prime}, \emptyset\right)$ can also be shown to satisfy the $F$-condition. By construction of $b_{i}\left(t_{i}^{\prime}, \emptyset\right)$, type $t_{i}^{\prime}$ satisfies condition A.1. Furthermore, $b_{i}\left(t_{i}^{\prime}, \emptyset\right)\left(s_{-i}, t_{-i}\right)>0$ only if $t_{-i}=t_{-i}^{s_{-i}, k-1}$ also by definition. Following the same steps as those used to prove $t_{i}^{k}$ satisfies condition T.1, type $t_{i}^{\prime}$ can also be shown to satisfy condition T.1.(a). Thus type $b_{i}\left(t_{i}^{k}, h\right)$ is the Bayesian updating of some type $t_{i}^{\prime}$ satisfying the $F$-condition and so satisfying condition T.2.(a).

By definition, type $t_{i}^{k}$ assigns positive probability only to opponents' profiles of types $t_{-i}^{s_{-i}, k-1}$. By construction, $b_{j}\left(t_{j}^{s_{-i}, k-1}, h\right) \in B_{j}^{k-1}(h)$ for any $j \neq i$. By the induction assumption, types 
$t_{j}^{s_{j}, k-1}$ express up to $(k-1)$-fold strong belief in $F$-rationality. Thus type $t_{i}^{k}$ assigns at every such $h$ positive probability only to opponents' type combinations expressing up to $(k-1)$-fold strong belief in $F$-rationality. Thus $t_{i}^{k}$ satisfies condition T.2 under Case (i).

Case (ii): There exists no such belief $b_{i}(\emptyset) \in B_{i}^{k}(\emptyset)$ with $b_{i}(\emptyset)\left(S_{-i}(h)\right)>0$. In this case

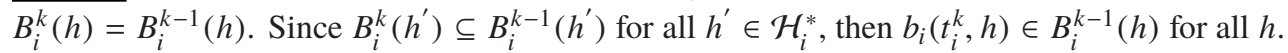
From the induction assumption $t_{i}^{k}$ expresses up to $(k-1)$-fold strong belief in $F$-rationality. For a type $t_{i}$ to expresses up to $k$-fold strong belief in $F$-rationality at an information set $h$ that can not be reached at the begining of the game, $b_{i}\left(t_{i}^{k}, h\right)$ must satisfy condition T.2 for the highest possible $l<k$. Since $b_{i}\left(t_{i}^{k}, h\right)$ satisfies T. 2 for $k-1$, type $t_{i}^{k}$ can be concluded to express up to $k$-fold strong belief in $F$-rationality.

\section{Proof of Lemma 3.1:}

We prove that $B_{i}^{k}(h)$ is the convex hull of finitely many points for every $h \in \mathcal{H}_{i}^{*}$ in three steps. First, we prove this is the case for $B_{i}^{0}(h)$ for all $h$. Subsequently, we proceed to prove this for $B_{i}^{k}(\emptyset)$ for any $k \geq 0$ by induction on $k$. Finally this is shown to be true for $B_{i}^{k}(h)$ for any $h \neq \emptyset$.

For any $h \in \mathcal{H}_{i}^{*}$, the set $B_{i}^{0}(h)$ is the convex hull of finitley many points:

Recall that $S_{i}$ is finite for all $i \in \mathcal{I}$ and that $B_{i}^{0}(h)=\Delta\left(S_{-i}(h)\right)$ for any $h \in \mathcal{H}_{i}^{*}$. It is therefore obvious that $B_{i}^{0}(h)$ is the convex hull of finitely many points.

The set $B_{i}^{k}(\emptyset)$ is the convex hull of finitely many points for any $k \geq 1$ :

This can be proven by induction on $k$. Assume $B_{i}^{k-1}(\emptyset)$ to be the convex hull of finitely many points. Consider the set $B_{i}^{k}(\emptyset)$. From the construction of the recursive procedure, $B_{i}^{k}(\emptyset)$ is characterized by the infinite set of linear inequalities

$$
b_{i}(\emptyset)\left(S_{-i}^{\varepsilon}\left(B_{-i}^{k-1}\right)\right) \geq F_{i}(\varepsilon) \text { for all } \varepsilon \geq 0
$$

It follows as a corollary from Lemma 3.2 in MPT (2013), that these restrictions can be equivalently characterized by a finite set of linear inequalities. Define the ascendingly ranked set $E=\left\{\varepsilon^{s_{j}}(k, \emptyset) \mid s_{j} \in S_{j}\right.$ for some $\left.j \neq i\right\}$ with cardinality $M$, where $\varepsilon^{s_{j}}(k, \emptyset)$ is the critical $\varepsilon$ of strategy $s_{j}$ in decision problem $\Gamma_{j}^{k}(\emptyset)$ and $M$ is finite. Let $\varepsilon_{-i}^{m}(k, \emptyset)$ be the $m^{\text {th }}$ element of set $E$.The finite set of linear inequalities characterizing $B_{i}^{k}(\emptyset)$ becomes

$$
b_{i}(\emptyset)\left(S_{-i}^{\varepsilon_{-i}^{m}}\left(B_{-i}^{k-1}\right)\right) \geq \underset{\varepsilon \uparrow \varepsilon_{-i}^{m+1}}{\operatorname{Lim}_{i}} F_{i}(\varepsilon) \quad \forall m \in\{1, \ldots, M-1\}
$$


Thus $B_{i}^{k}(\emptyset)$ is defined by finitely many linear restrictions on the convex set $B_{i}^{k-1}(\emptyset)$, making it the convex hull of finitely many points.

The set $B_{i}^{k}(h)$ is the convex hull of finitely many points for any $h \neq \emptyset$ :

Recall that $B_{i}^{k}(h):=\left\{b_{i}(h) \in B_{i}^{0}(h) \mid b_{i}(h)\left(s_{-i}\right)=\frac{b_{i}(\emptyset)\left(s_{-i}\right)}{b_{i}(\emptyset)\left(S_{-i}(h)\right)}\right.$ for every $s_{-i} \in S_{-i}(h)$ and for some $b_{i}(\emptyset) \in B_{i}^{k}(\emptyset)$ with $\left.b_{i}(\emptyset)\left(S_{j}(h)\right)>0\right\}$, as long as such $b_{i}(\emptyset)$ exists. Furthermore, $B_{i}^{k}(h)=$ $B_{i}^{k-1}(h)$ if there is no $b_{i}(\emptyset) \in B_{i}^{k}(\emptyset)$ with $b_{i}(\emptyset)\left(S_{j}(h)\right)>0$. Let $B_{i}^{k}(\emptyset)=\operatorname{conv}\left\{b_{i}^{1}, \ldots, b_{i}^{L}\right\}$ for $L<\infty$. Thus any $b_{i} \in B_{i}^{k}(\emptyset)$ can be written as

$$
b_{i}=\sum_{l=1}^{L} \alpha_{l} b_{i}^{l}
$$

Where $0 \leq \alpha_{l} \leq 1$ and $\sum_{l=1}^{L} \alpha_{l}=1$. Consequently,

$$
b_{i}\left(s_{-i}\right)=\sum_{l=1}^{L} \alpha_{l} b_{i}^{l}\left(s_{-i}\right)
$$

Take some $b_{i}^{l}$ and let $Y\left(b_{i}^{l}\right)$ be the Bayesian updating of $b_{i}^{l}$ on the set $S_{-i}(h)$, i.e. $Y\left(b_{i}^{l}\right) \in B_{i}^{k}(h)$. So for every $s_{-i} \in S_{-i}(h)$,

$$
Y\left(b_{i}^{l}\right)\left(s_{-i}\right)=\frac{b_{i}^{l}\left(s_{-i}\right)}{b_{i}^{l}\left(S_{-i}(h)\right)}
$$

Take some $b_{i}(h) \in B_{i}^{k}(h)$, then for every $s_{-i} \in S_{-i}(h)$ by definition of $B_{i}^{k}(h)$,

$$
b_{i}(h)\left(s_{-i}\right)=\frac{b_{i}(\emptyset)\left(s_{-i}\right)}{b_{i}(\emptyset)\left(S_{-i}(h)\right)} \quad \text { for some } b_{i}(\emptyset) \in B_{i}^{k}(\emptyset)
$$

Then $b_{i}(h)\left(s_{-i}\right)$ can be written as,

$$
\begin{aligned}
b_{i}(h)\left(s_{-i}\right) & =\frac{b_{i}(\emptyset)\left(s_{-i}\right)}{b_{i}(\emptyset)\left(S_{-i}(h)\right)}=\frac{\sum_{l=1}^{L} \alpha_{l} b_{i}^{l}\left(s_{-i}\right)}{\sum_{l=1}^{L} \alpha_{l} b_{i}^{l}\left(S_{-i}(h)\right)} \\
& =\sum_{l=1}^{L} \frac{\alpha_{l} b_{i}^{l}\left(S_{-i}(h)\right)}{\sum_{l=1}^{L} \alpha_{l} b_{i}^{l}\left(S_{-i}(h)\right)} \cdot Y\left(b_{i}^{l}\left(s_{-i}\right)\right)
\end{aligned}
$$


Let $\beta_{l}=\frac{\alpha_{l} b_{i}^{l}\left(S_{-i}(h)\right)}{\sum_{l=1}^{L} \alpha_{l} b_{i}^{l}\left(S_{-i}(h)\right)}$, then $b_{i}\left(s_{-i}\right)=\sum_{l=1}^{L} \beta_{l} \cdot Y\left(b_{i}^{l}\left(s_{-i}\right)\right.$, where $\sum_{l=1}^{L} \beta_{l}=1$.

Since the set $\left\{Y\left(b_{i}^{1}\right), \ldots, Y\left(b_{i}^{L}\right)\right\}$ is finite by the finiteness of $L$, it follows that any $b_{i} \in B_{i}^{k}(h)$ is a convex combination of finitely many points, i.e. $B_{i}^{k}(h)=\operatorname{conv}\left\{Y\left(b_{i}^{1}\right), \ldots, Y\left(b_{i}^{L}\right)\right\}$ for $L<\infty$. Note that the above proof assumes Bayesian updating at $h$ is possible. If that is not the case, then $B_{i}^{k}(h)=B_{i}^{k-1}(h)$, where $B_{i}^{k-1}(h)$ is the Bayesian updating of $B_{i}^{k-1}(\emptyset)$ if possible or is equal to some $B_{i}^{w}(h)$ where $w<k-1$ and where $B_{i}^{w}(h)$ is the Bayesian updating of $B_{i}^{w}(\emptyset)$. In both of these cases, the same steps of the proof guarantee that $B_{i}^{k}(h)$ is the convex hull of finitely many points. In case there exists no such $w \geq 1$ at which Bayesian updating of $B_{i}^{w}(\emptyset)$ is possible at $h$, then $B_{i}^{k}(h)=B_{i}^{0}(h)$, which is the convex hull of finitely many points as shown in the first part of this proof.

\section{Proof of Lemma 3.2:}

For a proof of Lemma 3.2, the reader is referred to the proof of Lemma 3.1 in the appendix of Mounir, Perea and Tsakas 2013. 


\section{Chapter 4}

\section{Reasoning Towards Common Belief in Reference Dependent Rationality}

\subsection{Introduction}

Reference dependent utility has been a topic of interest for the past decades, mainly within decision theoretic models but also to a growing extent in game theoretic contexts. Formalized by Khaneman and Tversky (1979) within their Prospect Theory, preferences were modeled over changes in levels of wealth relative to a certain reference point, mostly taken to be the status quo. In that context, the utility of each possible outcome of some decision or choice is compared to the reference point with losses weighting more heavily than gains of the same magnitude. Instead of the status-quo, the reference point was made endogenous and characterized recursively within a game context by Shalev (2000), thereby defining loss-aversion equilibrium. It is worth noting that he utility of a choice in Shalev's (2000) model consists of its classical expected utility and the expected loss associated with that choice relative to the reference point. In other words, gains received no positive weight in the gain-loss utility component. The reference point was allowed to be stochastic by Sugden (2003) where it was defined to be the player's potentially uncertain endowment in a decision theoretic context.

Motivated by experimental evidence indicating the role of expectations in forming players' reference point, Kőszegi and Rabin (2006) (henceforth KR06) defined their model of referencedependent utility where the reference point is defined by the player's expectations about his own choice as well as his belief about the environment. Under the KR06 model, a player evaluates a given choice by comparing each outcome to every outcome of the reference choice given the belief they hold about the uncertain environment they face. The reference choice in the KR06 model is the choice the player expects himself to play. Note that in this case players' expectations are assumed to be rational in the following sense; each player correctly anticipates the likelihood of changes in his environment and never expects himself to make a choice that he would not 
carry through with. Thus any choice is considered reference-dependent optimal if it is optimal for the player to stick to it once he anticipates playing it given the correct belief he holds about his environment. Note that the reference point in Sugden (2003) can also be interpreted, as he points out, as a reference choice within the player's choice set, thereby reinterpreting the model as one of expectation-based reference-dependent utility.

The models of Sugden (2003) and KR06 are both decision theoretic but could easily be extended to a multiplayer setting. In fact the KR06 model has been applied to many specific games, for example auctions (Lange and Ratan, 2010) and rank-order tournaments (Gill and Stone, 2010). Although a wide range of papers have applied reference-dependent utility models in specific games, contributions to formalizing expectation-based loss aversion in a general game theoretic context are still fairly limited. To our knowledge, only two papers in the literature addressed the matter in a general multiplayer setting, these are Shalev (2000) and Dato, Grunewald and Muller (2015), of which only the latter considered the reference point to be expectation-based. Moreover, all of these models are formulated within an equilibrium framework where each player is implicitly assumed to have correct beliefs about his opponents's choices and beliefs. Furthermore, the extent of players' sensitivity to losses and gains are assumed to be common knowledge.

This paper builds on the literature on expectation-based reference-dependent utility. While players in that literature are always assumed to hold correct beliefs about their opponents' choices and beliefs (in case of games) or more generally the distribution of the states of nature, this paper relaxes such assumption. Furthermore, the degree of sensitivity of each player to losses (and gains) in our model is, unlike the aforementioned literature, assumed to be each player's private information. The increased interactive uncertainty resulting from our departure from the equilibrium framework as well as the sensitivity coefficients of players being private information, makes it of great interest to formulate players beliefs within an epistemic framework. The concept of Common Belief in Reference Dependent Rationality (CBRDR) is therefore defined within an epistemic model, where reference dependent rationality (henceforth, RD-rationality) means players make those choices that maximize some reference-dependent utility (RD-utility) function given their belief about opponents' choices and their gain-loss sensitivity coefficients. Note that players' expectations are often assumed in the literature to be rational, i.e. players are assumed not to expect themselves making a choice not worth sticking to. This assumption is maintained throughout this paper, with the consequences of relaxing it addressed in the discussion section.

Throughout the paper, the game is viewed and analyzed from the perspective of one player $i$. The outcome of each choice of player $i$ is state dependent, where the combinations of opponents' choices serve as potential states of nature. Each player holds a belief or a probability distribution over the states of nature. The reference point is a combination of the player's belief and the 
choice he expects himself to play. Thus the reference point of any player $i$ is a probability distribution over the potential outcomes of player $i$ 's reference choice. Although the epistemic model we provide could allow for many forms of RD-utility functions, we focus in this paper on one such function. Based on his belief about the opponents' choices and an expectation of his own behavior, player $i$ in our model compares the outcome of every choice in each state with the outcome of the reference choice in the same state.The RD-utility function of interest is thus linear in beliefs and is closest to that of Sudden (2003). Note that our definition of the gain-loss component of the utility function is more restrictive than the one proposed by Sugden (2003), as we only consider cases where losses receive various positive weights while gains receive a weight of zero within the gain-loss component, thereby following the practice of Shalev (2000). However, the results of the paper are shown to generalize to the case where gains receive various positive weights as long as losses weight more heavily than gains of the same magnitude. A choice is made for such linear utility function because of its simplicity with the aim of using it as a benchmark to which other possibly nonlinear functions such as the one by Köszegi and Rabin (2006) can be compared, as shown in the discussion section.

One closely related stream of literature, especially to the specific RD-utility function considered in this paper, is that on regret-rejoice utility (Bell, 1982; Loomes and Sugden, 1982). Although different in their psychological intuition, these models have a functional form that is closely related to that of Sugden (2003), namely one in which each possible outcome of a given choice is compared to corresponding outcomes of other choices in the same state of nature. Just like our RD-utility function, outcomes are compared across choices for every state of nature and not across states of nature within each choice. This is unlike Kőszegi and Rabin (2006) where both comparisons are considered. The crucial difference between regret-rejoice models and reference-dependent ones remains in the psychological motivation of these models. In regretrejoice models the player compares each choice to every other choice in each state of nature he deems possible, i.e. there is no fixed reference choice relative to which all choice are evaluated. Furthermore, models on regret aversion do not necessarily assume a difference in the intensity with which regret is experienced as opposed to rejoice.

Models described so far, including the one introduced in this paper, implicitly assume sufficient time between the time the player is first confronted with the game and the moment of making a choice. Such elapsed time has to be sufficient for a certain choice to become a reference choice. In our model, the deliberation process is assumed to be elaborate enough to allow for players to form expectations about the choice they are about to make. Such expectations along with the players' beliefs about opponents' choices serve as the reference point each player evaluates all his choices relative to. An alternative approach to reference-dependent utility models implies players first commit to a certain choice which then becomes the reference choice relative to 
which gain and loss sensations are realized once the uncertainty of the game is resolved. Such ex post gain and loss sensations are then in turn accounted for ex ante by the player in the process of making his choice. One established example of that approach to reference-dependent utility, is the model of Köszegi and Rabin (2007) where the RD-utility of a choice involves comparing every possible outcome to each other outcome of the same choice, i.e. outcomes of each choice are compared amongst each other but not across choices. Hence, in that context each choice serves as its own reference point along with the belief the player holds about his opponents.

A similar intuition can be found in models of disappointment and elation such as Bell (1985); Loomes and Sugden (1986) and Gul (1991). The disappointment-elation component of utility in the models of Bell (1985) and Loomes and Sugden (1986) depends on how each possible outcome of the choice compares to the expected utility of that choice given the player's belief. Gul (1991) in turn differs from Bell (1985) and Loomes and Sugden (1986) in that each outcome of a given choice is evaluated relative to the expected modified utility itself. In other words, the modified utility of any choice is defined recursively, an approach adopted within referencedependent utility models by Shalev (2000). Note that the reference point is stochastic and endogenous in the Kószegi and Rabin (2007) model; endogenous but not stochastic in Bell (1985) and Loomes and Sugden (1986); and endogenous, recursive but not stochastic in the model of Gul (1991). Furthermore, despite being intuitively similar, these models remain behaviorally distinct (Masatlioglu and Raymond, 2014).

Once the epistemic model is defined, an algorithm characterizing Common Belief in Reference Dependent Rationality with the linear RD-utility function of choice is introduced. We prove that CBRDR where players preferences can be represented by our RD-utility function of choice and the gain-loss parameters are private information can be characterized by the algorithm of Iterated Elimination of RD-dominated Choices (IERDC) also known in the literature as Iterated Elimination of Börger- dominated Choices (Börgers, 1993). Hence, we also show that the choices that could be made under CBRDR where players preferences can be represented by our linear RD-utility function and with incomplete information about gain-loss parameters are identical to the choices that players can reasonably be expected to make under Common Belief in Ordinal Preferences over Pure Outcomes and rationality given some von-Neuman Morgenstern utility function consistent with player's ordinal preferences (Duggan and Le Breton, 2014). Although various RD-utility functions in the literature share a similar qualitative interpretation, it remains unclear how these different forms relate to one another. Therefore, implications of our characterization result for another RD-utility function, namely that of KR06, are considered. It is shown that the set of choices that can be made by players under CBRDR where players preferences can be represented by the KR06 RD-utility function are always included in those surviving Iterated Elimination of Börger- dominated Choices. 
In addition to being a handy computational tool characterizing CBRDR, the algorithm is also reinterpreted as a process of deliberation the players go through in an attempt to specify for themselves which choices they could expect their opponents as well as themselves to play under CBRDR. By considering it within the framework of Cubitt and Sugden (2011), the algorithm is interpreted as a process of deliberation players engage in as a step towards forming their expectations and beliefs about themselves and their opponents respectively. A similar contribution within epistemic game theory in that regard is that of Perea (2015) where the algorithm characterizing Common Belief in Future Rationality is interpreted as a reasoning procedure of finite steps that players go through to form their beliefs about their opponents' strategies.

The paper is structured as follows. Section 4.6 defines the notion of RD-rationality and constructs the concept of common belief in RD-rationality within an epistemic model. Section 4.3 shows how the notion of Bórgers dominance characterizes those choices that are RD-optimal for some belief and some loss-aversion coefficient under the linear RD-utility function of choice. While Section 4.4 reconstructs the algorithm of Iterated Elimination of Börger- dominated Choices in our context, Section 4.5.1 reinterprets the algorithm as a reasoning procedure of finite steps. Section 4.6 discusses, among others, the extension of the characterization result of this paper to the case where gains as well as losses receive positive weights and the implications of our results for the RD-utility of KR06.

\subsection{Common Belief in Reference Dependent-Rationality}

This section starts by defining the utility function of interest in subsection 4.2.1. Subsection 4.2.2 then uses a finite epistemic model to formalize Common Belief in Reference DependentRationality. Finally, subsection 4.2.3 illustrates the epistemic model by means of an example.

\subsubsection{Reference-Dependent Utility}

Consider an $n$-player finite normal form game $\Gamma=(\mathcal{I}, C, V)$, where $\mathcal{I}=\{1, \ldots, n\}$ is the finite set of players, $C=\left(C_{1}, \ldots, C_{n}\right)$ is an $n$-tuple of finite sets of choices and $V=\left(v_{1}, \ldots, v_{n}\right)$ where $v_{i}: C_{i} \times C_{-i} \rightarrow \mathfrak{R}$ is the utility function of player $i$. Let $b_{i} \in \Delta\left(C_{-i}\right)$ be a belief player $i$ holds about his opponents' choice combinations, where $\Delta\left(C_{-i}\right)$ is the set of probability distributions on $C_{-i}$. The subjective expected utility $v_{i}\left(c_{i}, b_{i}\right)$ of choice $c_{i} \in C_{i}$ is the utility of that choice 
given player $i$ 's belief $b_{i}$ about his opponents' choices, i.e.,

$$
v_{i}\left(c_{i}, b_{i}\right):=\sum_{c_{-i} \in C_{-i}} b_{i}\left(c_{-i}\right) v_{i}\left(c_{i}, c_{-i}\right)
$$

A reference point is characterized by a belief about the opponents' choice combinations and an expectation of one's own choice in response to such belief. For any player $i$, let $c_{i}^{*}$ be the reference choice relative to which player $i$ evaluates all his choices given his belief. The reference point is thus stochastic in the sense of Kószegi and Rabin (2006) with the probability distribution over outcomes being the result of the player's belief about his opponents' choices and his expectation of his own choice of actions. The main focus of this paper is the following reference-dependent (RD)-utility function based on Sugden (2003),

$$
u_{i}\left(c_{i}, b_{i}, c_{i}^{*}\right):=\sum_{c_{-i} \in C_{-i}} b_{i}\left(c_{-i}\right)\left[v_{i}\left(c_{i}, c_{-i}\right)-\lambda_{i}\left[v_{i}\left(c_{i}^{*}, c_{-i}\right)-v_{i}\left(c_{i}, c_{-i}\right)\right]_{+}\right]
$$

The second term in the right hand side of (1) is the gain-loss component of the utility function and satisfies the properties introduced by Kahneman and Tversky (1979) and formalized by Köszegi and Rabin (2006). It is defined for every $c_{-i} \in C_{-i}$ by

$$
\left[v_{i}\left(c_{i}^{*}, c_{-i}\right)-v_{i}\left(c_{i}, c_{-i}\right)\right]_{+}=\max \left\{\left(v_{i}\left(c_{i}^{*}, c_{-i}\right)-v_{i}\left(c_{i}, c_{-i}\right)\right), 0\right\}
$$

The parameter $\lambda_{i}$ is the loss aversion parameter of player $i$ and is strictly greater than 0 if player $i$ is loss averse. Note that $\lambda_{i}=0$ if the player is loss-neutral. In the latter case, $u_{i}\left(c_{i}, b_{i}, c_{i}^{*}\right)=v_{i}\left(c_{i}, b_{i}\right)$ for all $c_{i} \in C_{i}$. Moreover, for any reference choice $c_{i}^{*}$, it is always the case for choice $c_{i}^{*}$ that $u_{i}\left(c_{i}^{*}, b_{i}, c_{i}^{*}\right)=v_{i}\left(c_{i}^{*}, b_{i}\right)$ by definition of the reference-dependent utility function. From this point on, $\lambda_{i}$ will be included as an argument of the reference-dependent utility function $u_{i}\left(c_{i}, b_{i}, c_{i}^{*}, \lambda_{i}\right)$.

In this paper we assume that $\lambda_{i}$ is every player $i$ 's private information. The game of interest therefore becomes one of incomplete information. Let $\Gamma^{R D}=(\mathcal{I}, C, U, \Lambda)$ be a game of incomplete information, where $\mathcal{I}$ and $C$ are as defined before. Define $U=\left\{u_{1}, \ldots, u_{n}\right\}$ where $u_{i}: C_{i} \times C_{-i} \times C_{i} \times \Lambda_{i} \rightarrow \mathbb{R}$ is the utility function of player $i$. Moreover, let $\Lambda=\left\{\Lambda_{1}, \ldots, \Lambda_{n}\right\}$ where $\Lambda_{i}=[0, \infty)$ is the set of possible loss aversion coefficients for player $i$.

\subsubsection{The Epistemic Model}

In this section, we use a finite epistemic model to define common belief in reference dependent rationality. Note that this choice is without loss of generality as all the results obtained for the 
finite case are easily extendable to the infinite case. A finite epistemic model is formally defined in Definition 4.1.

Definition 4.1 A finite epistemic model for the game $\Gamma^{R D}$ is a tuple $M=\left(T_{i}, \lambda_{i}, b_{i}\right)_{i \in \mathcal{I}}$, where

- $T_{i}$ is a finite set of types of player $i$

- The function $\lambda_{i}: T_{i} \rightarrow[0, \infty)$ assigns a loss aversion coefficient $\lambda_{i}\left(t_{i}\right)$ to every $t_{i}$.

- The function $b_{i}: T_{i} \rightarrow \triangle\left(C_{-i} \times T_{-i}\right)$ assigns to each type $t_{i}$ a probability distribution over opponents' choice-type combinations.

Thus Definition 4.1 establishes that $\lambda_{i}\left(t_{i}\right)$ is a function of the type of player $i$. This is reflected in Definition 4.2 which defines the RD-utility of a choice-type combination $\left(c_{i}, t_{i}\right)$. Note that the player in our model is assumed to account for losses alone in comparing every choice to the reference point. In a more general context, positive weights could also assigned to gains. In such case a coefficient of sensitivity to gains can be constructed for every type in a similar manner as the loss aversion coefficients.

Definition 4.2 Utility of a choice-type combination $\left(c_{i}, t_{i}\right)$ for a reference choice $c_{i}^{*}$ is as follows

$$
u_{i}\left(c_{i}, t_{i}, c_{i}^{*}\right)=\sum_{\left(c_{-i}, t_{-i}\right) \in C_{-i} \times T_{-i}} b_{i}\left(t_{i}\right)\left(c_{-i}, t_{-i}\right)\left[v_{i}\left(c_{i}, c_{-i}\right)-\lambda_{i}\left(t_{i}\right)\left[v_{i}\left(c_{i}^{*}, c_{-i}\right)-v_{i}\left(c_{i}, c_{-i}\right)\right]_{+}\right]
$$

Now that the RD-utility of a choice-type combination is defined, Definition 4.3 specifies what it means for a a choice $c_{i}^{*}$ to be RD-optimal for some type $t_{i}$.

Definition 4.3 Choice $c_{i}^{*}$ is reference-dependent (RD)-optimal for type $t_{i}$, if $u_{i}\left(c_{i}^{*}, t_{i}, c_{i}^{*}\right) \geq$ $u_{i}\left(c_{i}, t_{i}, c_{i}^{*}\right)$ for all $c_{i} \in C_{i}$.

The above definition of RD-optimality is consistent with that adopted by Köszegi and Rabin (2006) in the sense that the player's expectation of his own choice, i.e. his reference choice, has to be rational. This assumption is maintained here, but relaxed in Section 4.6. It is worth noting that if a choice $c_{i}^{*}$ is RD-optimal for some type $t_{i}$ with a loss aversion coefficient $\lambda_{i}\left(t_{i}\right)$, it is also RD-optimal for a type $t_{i}^{\prime}$ with $b_{i}\left(t_{i}^{\prime}\right)=b_{i}\left(t_{i}\right)$ and for which $\lambda_{i}\left(t_{i}^{\prime}\right)>\lambda_{i}\left(t_{i}\right)$. For any given epistemic model, the set $R D_{i}$ of RD-optimal choice-type combinations of player $i$ is defined as follows.

Definition 4.4 The set of RD-optimal choice-type combinations $\left(c_{i}, t_{i}\right)$ can be defined as follows

$$
R D_{i}:=\left\{\left(c_{i}^{*}, t_{i}\right) \in C_{i} \times T_{i} \mid u_{i}\left(c_{i}^{*}, t_{i}, c_{i}^{*}\right) \geq u_{i}\left(c_{i}, t_{i}, c_{i}^{*}\right) \quad \forall c_{i} \in C_{i}\right\}
$$


Let $T_{i}^{1}$ be the set of types $t_{i}$ of player $i$ that believe in the opponents' RD-rationality. A type $t_{i}$ of player $i$ believes in his opponents' RD-rationality, i.e. $t_{i} \in T_{i}^{1}$, if it only assigns positive probability to opponents' choice-type combinations $\left(c_{-i}, t_{-i}\right)$ where for each $j \neq i$ choice $c_{j}$ is RD-optimal for type $t_{j}$ as in Definition 4.3. In other words, type $t_{i}$ assigns positive probability only to $\left(c_{-i}, t_{-i}\right) \in R D_{-i}$. Definition 4.5 formally introduces set $T_{i}^{1}$.

Definition 4.5 The set of types $t_{i}$ of player $i$ that believe in the opponents' RD-rationality is defined as

$$
T_{i}^{1}=\left\{t_{i} \in T_{i} \mid b_{i}\left(t_{i}\right)\left(R D_{-i}\right)=1\right\}
$$

A type $t_{i} \in T_{i}^{1}$ believing in the opponents' RD-rationality can be equivalently said to express one fold belief in RD-rationality. Type $t_{i}$ expresses 2-fold belief in RD-rationality, i.e. $t_{i} \in T_{i}^{2}$, if it assigns positive probability only to opponents' type combinations $t_{-i}$ where for every $j \neq i$, type $t_{j}$ believes in the opponents' RD-rationality, i.e where $t_{-i} \in T_{-i}^{1}$. More generally, type $t_{i}$ expresses $k$-fold belief in RD-rationality for $k \geq 2$, i.e. $t_{i} \in T_{i}^{k}$, if it assigns positive probability only to opponents' type combinations $t_{-i} \in T_{-i}^{k-1}$ expressing $k-1$-fold belief in RD-rationality. Furthermore, type $t_{i}$ expresses common belief in RD-rationality if it expresses $k$-fold belief in RD-rationality for every $k \geq 1$.

Definition 4.6 Type $t_{i}$ expresses $k$-fold belief in $R D$-rationality for $k \geq 2$, i.e. $t_{i} \in T_{i}^{k}$ if it assigns positive probability only to $t_{-i} \in T_{-i}^{k-1}$. Hence,

$$
T_{i}^{k}=\left\{t_{i} \in T_{i} \mid b_{i}\left(t_{i}\right)\left(C_{-i} \times T_{-i}^{k-1}\right)=1\right\} .
$$

The set of types $T_{i}^{\infty}$ expressing common belief in RD-rationality is defined by

$$
T_{i}^{\infty}=\bigcap_{k \geq 1} T_{i}^{k}
$$

Hence, a choice $c_{i}$ can be RD-rationally made under common belief in reference-dependent rationality given some loss-aversion coefficient $\lambda_{i} \geq 0$ whenever there exists an epistemic type $t_{i} \in T_{i}^{\infty}$ such that $\left(c_{i}, t_{i}\right)$ is RD-rational given $\lambda_{i}\left(t_{i}\right)=\lambda_{i}$.

\subsubsection{The Epistemic Model: An Example}

This subsection presents and illustrating example of the model introduced in subsection 4.2.2. Consider the two player game $\Gamma$ given in Table 4.1. For player 1, choice $M$ is strictly dominated in the game $\Gamma$ by a randomization $r_{1} \in \Delta\{U, D\}$ such that $\frac{1}{2}<r_{1}(U)<\frac{2}{3}$. All other choices of 
player 1 and those of player 2 are optimal for some belief in game $\Gamma$. Hence, Choice $M$ is not optimal for any belief if the player is a standard expected utility maximizer.

\begin{tabular}{c|cc}
\hline$\Gamma$ & $\mathrm{L}$ & $\mathrm{R}$ \\
\hline $\mathrm{U}$ & 7,7 & 4,6 \\
$\mathrm{M}$ & 5,6 & 5,7 \\
$\mathrm{D}$ & 3,7 & 7,8
\end{tabular}

TABLE 4.1: Original Game $\Gamma$

Consider the epistemic model $M$ assigning types sets $T_{1}=\left\{t_{1}^{a}, t_{1}^{b}, t_{1}^{c}, t_{1}^{d}\right\}$ and $T_{2}=\left\{t_{2}^{a}, t_{2}^{b}\right\}$ to players 1 and 2 respectively. Table 4.2 summarizes the type sets of the two players.

\begin{tabular}{c|c|c}
\hline \multicolumn{3}{c}{ Types of Player 1 } \\
\hline \hline$t_{1}^{a}$ & $b_{1}\left(t_{1}^{a}\right)=0.5\left(L, t_{2}^{a}\right)+0.5\left(R, t_{2}^{a}\right)$ & $\lambda_{1}\left(t_{1}^{a}\right)=2$ \\
$t_{1}^{b}$ & $b_{1}\left(t_{1}^{b}\right)=0.2\left(L, t_{2}^{a}\right)+0.8\left(R, t_{2}^{b}\right)$ & $\lambda_{1}\left(t_{1}^{b}\right)=2$ \\
$t_{1}^{c}$ & $b_{1}\left(t_{1}^{c}\right)=0.2\left(L, t_{2}^{a}\right)+0.8\left(R, t_{2}^{a}\right)$ & $\lambda_{1}\left(t_{1}^{c}\right)=3$ \\
$t_{1}^{d}$ & $b_{1}\left(t_{1}^{d}\right)=0.2\left(L, t_{2}^{b}\right)+0.8\left(R, t_{2}^{b}\right)$ & $\lambda_{1}\left(t_{1}^{d}\right)=2$ \\
\hline \multicolumn{3}{c}{ Types of Player 2 } \\
\hline \hline$t_{2}^{a}$ & $b_{2}\left(t_{2}^{a}\right)=0.7\left(U, t_{1}^{a}\right)+0.3\left(M, t_{1}^{c}\right)$ & $\lambda_{2}\left(t_{2}^{a}\right)=3$ \\
$t_{2}^{b}$ & $b_{2}\left(t_{2}^{b}\right)=0.7\left(D, t_{1}^{d}\right)+0.3\left(M, t_{1}^{b}\right)$ & $\lambda_{2}\left(t_{2}^{b}\right)=2$ \\
\hline
\end{tabular}

TABle 4.2: The Epistemic Model

Both choices $U$ and $D$ are RD-optimal for all four types of player 1, while choice $M$ is RDoptimal only for types $t_{1}^{a}$ and $t_{1}^{c}$. Note that under standard expected utility maximization, only choices $U$ and $D$ can be optimal for some type. As an example, we show that choice $M$ is RD-optimal for type $t_{1}^{c}$. Taking choice $M$ as the reference choice, then the reference dependent utilities of each choice of player 1 given type $t_{1}^{c}$ are as follows

$$
\begin{aligned}
u_{1}\left(U, t_{1}^{c}, M\right)= & 0.2\left[v_{1}(U, L)-\lambda_{1}\left(t_{1}^{c}\right)\left[v_{1}(M, L)-v_{1}(U, L)\right]_{+}\right]+ \\
& 0.8\left[v_{1}(U, R)-\lambda_{1}\left(t_{1}^{c}\right)\left[v_{1}(M, R)-v_{1}(U, R)\right]_{+}\right] \\
= & 2.2, \\
u_{1}\left(M, t_{1}^{c}, M\right)= & 0.2\left[v_{1}(M, L)\right]+0.8\left[v_{1}(M, R)\right] \\
= & 5, \\
u_{1}\left(D, t_{1}^{c}, M\right)= & 0.2\left[v_{1}(D, L)-\lambda_{1}\left(t_{1}^{c}\right)\left[v_{1}(M, L)-v_{1}(D, L)\right]_{+}\right]+ \\
& 0.8\left[v_{1}(D, R)-\lambda_{1}\left(t_{1}^{c}\right)\left[v_{1}(M, R)-v_{1}(D, R)\right]_{+}\right] \\
= & 5 .
\end{aligned}
$$


From the above utilities, it is clear that choice $M$ is RD-optimal for type $t_{1}^{c}$. In a similar manner, choice $L$ and $R$ of player 2 can be shown to be RD-optimal for $t_{2}^{a}$ of player 2, while given type $t_{2}^{b}$ only choice $R$ is RD-optimal. Recall that a type believes in the opponent's RD-rationality if it assigns positive probability only to $\left(c_{j}, t_{j}\right)$ where $c_{j}$ is RD-optimal for $t_{j}$. It is easy to check that except for type $t_{1}^{d}$, all types of player 1 believe in their opponent's RD-rationality. Thus we obtain the set $T_{1}^{1}=\left\{t_{1}^{a}, t_{1}^{b}, t_{1}^{c}\right\}$. For player 2, only type $t_{2}^{a}$ believes in the opponent's RD-rationality, i.e. $T_{2}^{1}=\left\{t_{2}^{a}\right\}$. Type $t_{2}^{b} \notin T_{2}^{1}$, because it assigns probability 0.3 to player 1 being of type $t_{1}^{b}$ and playing $M$ while the latter is not RD-optimal for type $t_{1}^{b}$.

Furthermore, a type $t_{i}$ expresses 2-fold belief in RD-rationality if it assigns positive probability only to opponents' types that believe in the opponents' RD-rationality. Thus types $t_{1}^{a}$ and $t_{1}^{c}$ of player 1 express 2-fold belief in RD-rationality, while type $t_{1}^{b}$ does not as it assigns a probability of 0.8 to type $t_{2}^{b} \notin T_{2}^{1}$. Type $t_{2}^{a}$ of player 2 also express 2-fold belief in RD-rationality, while $t_{2}^{b}$ does not. The set of types of each player expressing 2-fold belief in RD-rationality are thus $T_{1}^{2}=\left\{t_{1}^{a}, t_{1}^{c}\right\}$ and $T_{2}^{2}=\left\{t_{2}^{a}\right\}$ for players 1 and 2 respectively. These two sets of types can also be shown to express common belief in RD-rationality.

\subsection{Characterization of Reference Dependent Rational Choices}

The main result of this section is a characterization of the set of choices $C R D_{i}$ that are reference dependence optimal for some belief and some $\lambda_{i} \geq 0$ in any normal-form game $\Gamma$. Lemma 4.1 in subsection 4.3.1 shows that the set $C R D_{i}$ is equivalent to the set of choices that are not Börgersdominated (Börgers, 1993). Subsection 4.3.1 also presents some implications of Lemma 4.1 along with a characterization of choices that are RD-rational for some given $\lambda_{i}$. While the proof of Lemma 4.1 is detailed in the appendix, subsection 4.3.2 presents two examples to illustrate the main steps of the two directions of the proof.

\subsubsection{Characterization Result}

This section presents a characterization of the set $C R D_{i}$ of choices that are RD-optimal for some belief $b_{i} \in \Delta\left(C_{-i}\right)$ and some $\lambda_{i} \geq 0$. Define $C R D_{i}=\left\{c_{i}^{*} \in C_{i} \mid \exists \lambda_{i} \geq 0\right.$ and $b_{i} \in$ $\triangle\left(C_{-i}\right)$ s.t. $\left.u_{i}\left(c_{i}^{*}, b_{i}, c_{i}^{*}, \lambda_{i}\right) \geq u_{i}\left(c_{i}, b_{i}, c_{i}^{*}, \lambda_{i}\right) \forall c_{i} \in C_{i}\right\}$, where $u_{i}\left(c_{i}, b_{i}, c_{i}^{*}, \lambda_{i}\right)$ is as defined in equation (1) in section 4.2.1 above. Lemma 4.1 shows that a choice $c_{i}^{*}$ is RD-optimal for some belief $b_{i} \in \Delta\left(C_{-i}\right)$ and some $\lambda_{i} \geq 0$, i.e. $c_{i}^{*} \in C R D_{i}$, if and only if there is at least one subset $C_{-i}^{\prime} \subseteq C_{-i}$ of opponents' choice-combinations such that there is no choice $c_{i} \in C_{i}$ weakly dominating $c_{i}^{*}$ in the reduced game $\Gamma_{R}=\left(C_{i}, C_{-i}^{\prime} ; v_{i}, v_{-i}\right)$. 
Lemma 4.1 A choice $c_{i}^{*} \notin C R D_{i}$ if and only if for every subset $C_{-i}^{\prime} \subseteq C_{-i}$ there exists some $c_{i} \in C_{i}$ that weakly dominates $c_{i}^{*}$ in the reduced game $\Gamma_{R}=\left(C_{i}, C_{-i}^{\prime} ; v_{i}, v_{-i}\right)$.

Note that the notion of dominance utilized in Lemma 4.1 to characterize $C R D_{i}$ is identical to that of Definition 4 of Börgers (1993). Corollary 4.1 formalizes one implication of Lemma 4.1. It shows that a choice $c_{i}^{*}$ that is strictly dominated in game $\Gamma$ by some other choice $c_{i}$ is never RD-optimal for any belief and any $\lambda_{i} \geq 0$. This is an implication of Lemma 4.1 because choice $c_{i}$ in that case weakly dominates choice $c_{i}^{*}$ in all reduced games of the form $\Gamma_{R}=\left(C_{i}, C_{-i}^{\prime} ; v_{i}, v_{-i}\right)$.

Corollary 4.1 Let $c_{i}^{*}$ be a choice for which there exists some $c_{i} \in C_{i}$ such that $v_{i}\left(c_{i}^{*}, c_{-i}\right)<$ $v_{i}\left(c_{i}, c_{-i}\right)$ for every $c_{-i} \in C_{-i}$, then $c_{i}^{*} \notin C R D_{i}$.

While Lemma 4.1 characterizes the set $C R D_{i}$ of RD-rational choices of player $i$, it remains to characterize those choices $C_{i}^{1}\left(\lambda_{i}\right)$ of player $i$ that are RD-rational for some given $\lambda_{i}$. Let $\Gamma_{i}\left(c_{i}^{*}, \lambda_{i}\right)$ be a decision problem of player $i$ where choice $c_{i}^{*}$ serves as the reference choice and $\lambda_{i}$ is some given value of player $i$ 's loss-aversion coefficient. Moreover, define the payoffs of each choice $c_{i} \in C_{i}$ of player $i$ in the decision problem $\Gamma_{i}\left(c_{i}^{*}, \lambda_{i}\right)$ to be $u_{i}\left(c_{i}, c_{-i}\right)=v_{i}\left(c_{i}, c_{-i}\right)-$ $\lambda_{i}\left[v_{i}\left(c_{i}^{*}, c_{-i}\right)-v_{i}\left(c_{i}, c_{-i}\right)\right]_{+}$for each $c_{-i} \in C_{-i}$. Based on lemma 3 of Pearce (1984), lemma 4.2 below shows that a choice $c_{i}^{*}$ is RD-optimal for some belief $b_{i}$ and some given $\lambda_{i}$ if and only if it is not strictly dominated in the decision problem $\Gamma_{i}\left(c_{i}^{*}, \lambda_{i}\right)$.

Lemma 4.2 Choice $c_{i}^{*} \in C_{i}^{1}\left(\lambda_{i}\right)$ if and only if it is not strictly dominated by any randomization $r_{i} \in \Delta\left(C_{i}\right)$ in the decision problem $\Gamma_{i}\left(c_{i}^{*}, \lambda_{i}\right)$.

\subsubsection{Illustrating Examples of Lemma 4.1}

The proof of Lemma 4.1 consists of proving two directions. First, we show that for any choice $c_{i}^{*} \in C_{i}$ for which there exists some $c_{i} \in C_{i}$ for every subset $C_{-i}^{\prime} \subseteq C_{-i}$ such that $c_{i}^{*}$ is weakly dominated by $c_{i}$ in the reduced game $\Gamma_{R}=\left(C_{i}, C_{-i}^{\prime} ; v_{i}, v_{-i}\right)$, it must be that $c_{i}^{*} \notin C R D_{i}$. (part A). Second, If choice $c_{i}^{*} \notin C R D_{i}$ then it must be that for every subset $C_{-i}^{\prime} \subseteq C_{-i}$ there is some choice $c_{i} \in C_{i}$ that weakly dominates $c_{i}^{*}$ in the reduced game $\Gamma_{R}=\left(C_{i}, C_{-i}^{\prime} ; v_{i}, v_{-i}\right)$ (part B). This section uses two examples to illustrate the proof of those two parts.

The example illustrating part A of Lemma 4.1 uses game $\Gamma$ in Table 4.3. Note that the table shows only the payoffs of player 1 . The payoffs of the column player 2 have been omitted from the table as they are not needed for the purpose of this illustration. Note that choice $a$ in $\Gamma$ is not optimal for any belief $b_{1} \in \Delta\left(C_{2}\right)$ under standard expected utility maximization. Furthermore, for every $C_{2}^{\prime} \subseteq C_{2}$, there is a choice of player 1 weakly dominating $a$ in the reduced game 
$\Gamma_{R}=\left(C_{1}, C_{2}^{\prime} ; v_{1}, v_{2}\right)$. Note that $a$ is weakly dominated by $b$ on every $C_{2}^{\prime}$ with $C_{2}^{\prime} \cap\{e\} \neq \emptyset$, by $c$ on $C_{2}^{\prime}=\{f, g\}$ and by $d$ on $C_{2}^{\prime}=\{g\}$. To evaluate whether $a$ is RD-optimal for some $\lambda>0$, the optimality of $a$ is checked in the decision problem of Table 4.4.

\begin{tabular}{c|ccc}
\hline$\Gamma$ & $e$ & $f$ & $g$ \\
\hline$a$ & 1 & 1 & 1 \\
$b$ & 2 & 1 & 1 \\
$c$ & 0 & 4 & 1 \\
$d$ & 0 & 0 & 4 \\
\hline
\end{tabular}

TABle 4.3: Game $\Gamma$

\begin{tabular}{c|ccc}
\hline$\Gamma(a, \lambda)$ & $e$ & $f$ & $g$ \\
\hline$a$ & 1 & 1 & 1 \\
$b$ & 2 & 1 & 1 \\
$c$ & $-\lambda$ & 4 & 1 \\
$d$ & $-\lambda$ & $-\lambda$ & 4 \\
\hline
\end{tabular}

Table 4.4: Game $\Gamma(a, \lambda)$

It is easy to verify from decision problem $\Gamma(a, \lambda)$ that for every $b_{1} \in \Delta\left(C_{2}\right)$ there is some choice of player 1 other than $a$ that is optimal for that belief regardless of the value of $\lambda$. Consider for example a belief $b_{1} \in \Delta(\{f, g\})$ where both $b_{1}(f)>0$ and $b_{1}(g)>0$. Then given $b_{1}$ and any $\lambda>0, u\left(c, b_{1}, a, \lambda\right)>u\left(a, b_{1}, a, \lambda\right)$. Therefore, $a$ can not be RD-optimal for such $b_{1}$ for any $\lambda>0$. Similarly for a belief $b_{1}^{\prime} \in \Delta(\{e, g\})$ where both $b_{1}^{\prime}(e)>0$ and $b_{1}^{\prime}(g)>0$. Given $b_{1}^{\prime}$, $u\left(b, b_{1}, a, \lambda\right)>u\left(a, b_{1}, a, \lambda\right)$ for any $\lambda>0$. A similar argument can be made for every belief $b_{1}^{*} \in \Delta\left(C_{2}^{\prime}\right)$ where $C_{2}^{\prime} \subseteq C_{2}$. Thus choice $a$ cannot be RD-optimal for any belief given any $\lambda>0$.

The second example is of game $\Gamma^{\prime}$ presented in Table 4.5 and is used to illustrate part B of the proof of Lemma 4.1. The proof shows that a choice such as $a$ in this example is RD-optimal for some belief and some $\lambda^{a} \geq 0$. Note that in game $\Gamma^{\prime}$, choice $a$ is weakly dominated in the reduced games defined by $\Gamma_{R}^{\prime}=\left(C_{1}, C_{2}^{\prime} ; v_{1}, v_{2}\right)$, for $C_{2}^{\prime} \in\{\{e, f, g\},\{e\},\{f\},\{g\},\{e, g\},\{e, f\}\}$ by choices $b, b, c, d, b$ and $b$ respectively. However, choice $a$ is not weakly dominated by any choice in the reduced game $\Gamma_{R}^{\prime}=\left(C_{1},\{f, g\} ; v_{1}, v_{2}\right)$.

\begin{tabular}{c|ccc}
\hline$\Gamma^{\prime}$ & $e$ & $f$ & $g$ \\
\hline$a$ & 1 & 1 & 1 \\
$b$ & 2 & 1 & 1 \\
$c$ & 0 & 4 & 0 \\
$d$ & 0 & 0 & 4 \\
\hline
\end{tabular}

TABle 4.5: Game $\Gamma^{\prime}$

\begin{tabular}{c|ccc}
\hline$\Gamma^{\prime}(a, \lambda)$ & $e$ & $f$ & $g$ \\
\hline$a$ & 1 & 1 & 1 \\
$b$ & 2 & 1 & 1 \\
$c$ & $-\lambda$ & 4 & $-\lambda$ \\
$d$ & $-\lambda$ & $-\lambda$ & 4 \\
\hline
\end{tabular}

TABLE 4.6: Game $\Gamma^{\prime}(a, \lambda)$

Note that choice $a$ is not optimal for any belief in $\Gamma^{\prime}$ for $\lambda=0$ as it is strictly dominated by a randomization $r \in \Delta(\{b, c, d\})$, where $r(b)=0.54$ and $r(c)=r(d)=0.23$. In what follows, we show that the fact that there is no choice weakly dominating $a$ in the reduced game $\Gamma_{R}^{\prime}=\left(C_{1},\{f, g\} ; v_{1}, v_{2}\right)$ will allow choice $a$ to be RD-optimal for some belief $b_{1} \in \Delta(\{f, g\})$ for some $\lambda>0$. 
Choice $a$ is RD-rational for some $\lambda>0$ if it is optimal for some belief in the decision problem $\Gamma^{\prime}(a, \lambda)$ of Table 4.6. Consider some $b_{1} \in \Delta(\{f, g\})$ where both $b_{1}(f)>0$ and $b_{1}(g)>0$, $b_{1}(f)=b_{1}(g)=0.5$ for instance. The RD-utility of each choice taking $a$ as reference choice, given $b_{1}$ and as a function of $\lambda>0$ are as follows: $u\left(a, b_{1}, a, \lambda\right)=u\left(b, b_{1}, a, \lambda\right)=1$ and $u\left(c, b_{1}, a, \lambda\right)=u\left(d, b_{1}, a, \lambda\right)=2-0.5 \lambda$. It suffices to show that there is some $\lambda>0$ such that $u\left(a, b_{1}, a, \lambda\right) \geq u\left(c, b_{1}, a, \lambda\right)$. Note that the latter is satisfied for any $\lambda \geq 2$.

\subsection{Iterated Elimination of RD-dominated Choices (IERDC)}

This section establishes that the iteration of the dominance notion due to Börgers (1993) characterizes those choices that players can be expected to choose under CBRDR. The algorithm is reconstructed here under the title of Iterated Elimination of RD-dominated Choices (IERDC). The basic model of this paper assumes players to be RD-rational for some loss-aversion coefficient the value of which is each player's private information. Each player $i$ can thus only be expected to play choices $c_{i}$ that are RD-optimal for some belief $b_{i}$ and for some $\lambda_{i} \geq 0$, i.e. $c_{i} \in C R D_{i}$. Characterizing the set $C R D_{i}$ of choices that are not RD-dominated in $\Gamma$ is the first round of Algorithm 4.1. Set $C R D_{i}$, or as denoted henceforth $C R D_{i}^{1}$, is defined for every player $i$ by $C R D_{i}^{1}=\left\{c_{i}^{*} \in C_{i} \mid \exists \lambda_{i} \geq 0\right.$ and $b_{i} \in \triangle\left(C_{-i}\right)$ s.t. $\left.u_{i}\left(c_{i}^{*}, b_{i}, c_{i}^{*}, \lambda_{i}\right) \geq u_{i}\left(c_{i}, b_{i}, c_{i}^{*}, \lambda_{i}\right) \forall c_{i} \in C_{i}\right\}$ and is characterized by Börgers- dominance as shown in Lemma 4.1.

Round 2 of the algorithm defines set $C R D_{i}^{2}$ for every player $i$, which is the set of choices $c_{i}$ that are RD-optimal for some belief $b_{i}$ and for some $\lambda_{i} \geq 0$ in the modified game $\Gamma^{1}=\left(\mathcal{I}, C R D^{1}, V\right)$. From Definitions 4.4 and 4.5, an epistemic type $t_{i}$ of player $i$ expresses 1-fold belief in RDrationality if it assigns positive probability only to opponents' choice-type combinations $\left(c_{-i}, t_{-i}\right)$ where the choice of every opponent is RD-optimal for the belief of the associated type and given the loss aversion coefficient of that type. Thus believing in the opponents' RD-rationality, player $i$ assigns positive probability only to opponents' choice-type $\left(c_{-i}, t_{-i}\right)$ combinations where $c_{-i} \in C R D_{-i}^{1}$.

The set $C R D_{i}^{k}=\left\{c_{i}^{*} \in C R D_{i}^{k-1} \mid \exists \lambda_{i} \geq 0\right.$ and $b_{i} \in \Delta\left(C R D_{-i}^{k-1}\right)$ s.t. $u_{i}\left(c_{i}^{*}, b_{i}, c_{i}^{*}, \lambda_{i}\right) \geq$ $\left.u_{i}\left(c_{i}, b_{i}, c_{i}^{*}, \lambda_{i}\right) \forall c_{i} \in C_{i}\right\}$ of choices is characterized for player $i$ by round $k$ of the algorithm, based on Lemma 4.1 and through the following steps. Recall from Lemma 4.1 that $c_{i}^{*}$ is RDoptimal for some $b_{i}$ and some $\lambda_{i}$ if and only if there exists some $C_{-i}^{\prime} \subseteq C_{-i}$ such that there is no $c_{i}^{\prime} \in C_{i}$ weakly dominating $c_{i}^{*}$ in $\Gamma_{R}\left(C_{-i}^{\prime}\right)=\left(C_{i}, C_{-i}^{\prime} ; v_{i}, v_{-i}\right)$. Based on $\Gamma^{k-1}=\left(I, C R D^{k-1}, V\right)$ being defined, step A.1 of Algorithm 4.1 defines the set $C R D_{i}^{k}$ for every player $i$ as follows

$$
C R D_{i}^{k}=\left\{c_{i}^{*} \in C R D_{i}^{k-1} \mid \exists C_{-i}^{\prime} \subseteq C R D_{-i}^{k-1} \text { s.t. } \exists c_{i}^{\prime} \text { weakly dominating } c_{i}^{*} \text { in } \Gamma_{R}^{k-1}\left(C_{-i}^{\prime}\right)\right\} .
$$


The sets $C R D_{i}^{k}$ for each player $i$ are then used in step A.2 to define the modified game $\Gamma^{k}=$ $\left(I, C R D^{k}, V\right)$ upon which the next round of the algorithm will be based. The procedure terminates after round $K$ when $C R D_{i}^{K}=C R D_{i}^{K+1}$ for all $i$ and the resulting set for each player $i$ is denoted by $C R D_{i}^{K}$. The steps of Algorithm 4.1 are formalized below.

Algorithm 4.1 Initial Step: Define $\Gamma^{0}=(I, C, V)$ where $C=C R D^{0}$.

Inductive Step: Assume $\Gamma^{k-1}$ has been defined. Then

A.1 Define for every player $i$ the set

$$
C R D_{i}^{k}=\left\{c_{i}^{*} \in C R D_{i}^{k-1} \mid \exists C_{-i}^{\prime} \subseteq C R D_{-i}^{k-1} \text { s.t. } \nexists c_{i}^{\prime} \text { weakly dominating } c_{i}^{*} \text { in } \Gamma_{R}^{k-1}\left(C_{-i}^{\prime}\right)\right\} .
$$

\section{A.2 Define game $\Gamma^{k}=\left(\mathcal{I}, C R D^{k}, V\right)$}

Theorem 4.1 shows that Algorithm 4.1 characterizes for every $k \geq 0$ and every player $i$ exactly those choices $C R D_{i}^{k+1}$ that could be made by an epistemic type $t_{i}$ expressing up to $k$-fold belief in RD-rationality. Since Theorem 4.1 shows that this holds for all $k \geq 0$, it also proves that the final set produced by Algorithm 4.1, i.e. $C R D_{i}^{K}$, is exactly the set of choices that are RD-optimal for some type $t_{i}$ of player $i$ expressing common belief in RD-rationality.

Theorem 4.1 A choice $c_{i}$ of player $i$ can be RD-optimal for some $t_{i}$ expressing up to $k$-fold belief in RD-rationality if and only if choice $c_{i}$ survives $k+1$ rounds of Algorithm 4.1, i.e. $c_{i} \in C R D_{i}^{k+1}$.

Notably, iterated elimination of Börgers- dominated choices has also been proven to characterize Common Belief in Ordinal Preferences over Pure Outcomes and rationality given some vonNeuman Morgenstern utility function consistent with those preferences (Duggan and Le Breton, 2014). Under the latter, players' utility functions are not exogenously given, instead only players' ordinal preferences over pure outcomes of choices are commonly believed in. A player can then be rationally expected to make any choice that maximizes a utility function consistent with his ordinal preferences over pure outcomes. Thus theorem 4.1 also implicates an equivalence between Common Belief in Ordinal Preferences over Pure Outcomes and rationality and CBRDR where players preferences can be represented by our linear RD-utility function and with incomplete information about players' loss-aversion coefficients.

Now consider the case of $\lambda_{i}$ being common knowledge for every player $i$. Using Lemma 4.2, Algorithm 4.2 is defined to characterize for every $i$ the set of choices $C_{i}^{k}\left(\lambda_{i}\right)$ that could be made under up to $k$-fold belief in RD-rationality for every $k \geq 1$. Define the game $\Gamma^{0}=\left(\mathcal{I}, C^{0}, \lambda, V\right)$ where $\mathcal{I}$, and $V$ are as defined before, $C^{0}=\left(C_{1}, \ldots, C_{n}\right)$ and $\lambda=\left(\lambda_{i}, \ldots, \lambda_{n}\right)$. Vector $\lambda$ is common 
knowledge. Round 1 of the algorithm characterizes for every player $i$ the set $C_{i}^{1}\left(\lambda_{i}\right)$ of choices $c_{i} \in C_{i}$ that are RD-optimal for some belief $b_{i}$ and given $\lambda_{i}$, i.e. $C_{i}^{1}\left(\lambda_{i}\right):=\left\{c_{i} \in C_{i}^{0} \mid \nexists r_{i} \in\right.$ $\triangle\left(C_{i}^{0}\right)$ s.t. $\left.u_{i}\left(r_{i}, c_{-i}, c_{i}, \lambda_{i}\right)>u_{i}\left(c_{i}, c_{-i}, c_{i}, \lambda_{i}\right) \forall c_{-i} \in C_{-i}^{0}\right\}$. Similarly, round $k$ of Algorithm 4.2 starts from game $\Gamma^{k-1}=\left(\mathcal{I}, C^{k-1}(\lambda), \lambda, V\right)$ and characterizes for every player $i$ the set $C_{i}^{k}\left(\lambda_{i}\right):=\left\{c_{i} \in C_{i}^{k-1}\left(\lambda_{i}\right) \mid \nexists r_{i} \in \Delta\left(C_{i}^{k-1}\right)\right.$ s.t. $\left.u_{i}\left(r_{i}, c_{-i}, c_{i}, \lambda_{i}\right)>u_{i}\left(c_{i}, c_{-i}, c_{i}, \lambda_{i}\right) \forall c_{-i} \in C_{-i}^{k-1}\right\}$.

Algorithm 4.2 Initial Step: Define $\Gamma^{0}=\left(\mathcal{I}, C^{0}, \lambda, V\right)$

Inductive Step: Assume $\Gamma^{k-1}$ has been defined. Then

B.1 For every player $i$, define the set

$$
\begin{aligned}
& C_{i}^{k}\left(\lambda_{i}\right):=\left\{c_{i} \in C_{i}^{k-1}\left(\lambda_{i}\right) \mid \nexists r_{i} \in \Delta\left(C_{i}^{k-1}\left(\lambda_{i}\right)\right) \text { s.t. } u_{i}\left(r_{i}, c_{-i}, c_{i}, \lambda_{i}\right)>u_{i}\left(c_{i}, c_{-i}, c_{i}, \lambda_{i}\right)\right. \\
& \left.\forall c_{-i} \in C_{-i}^{k-1}\right\} .
\end{aligned}
$$

B.2 Define game $\Gamma^{k}=\left(\mathcal{I}, C^{k}(\lambda), \lambda, V\right)$

Given the purpose of the two procedures defined above, it seems natural to expect that the choices surviving Algorithm 4.2 are always a subset of those surviving Algorithm 4.1. Lemma 4.3 shows that this is in fact true for every $k \geq 0$.

Lemma 4.3 For every player $i$ and for any loss-aversion coefficient $\lambda_{i}$,

$$
C_{i}^{k}\left(\lambda_{i}\right) \subseteq C R D_{i}^{k}
$$

As shown by Apt (2011), Algorithm 4.1, also referred to as Iterated Börgers Dominance or Iterated Inherent Dominance, is order independent. Thus it makes no difference to the end result, i.e. sets $C R D_{i}^{K}$ for every player $i$, if in some round $k$ of Algorithm 4.1 only some but not all choices $c_{i} \in C R D_{i}^{k-1} \backslash C R D_{i}^{k}$ are eliminated. Theorem 4.2 restates this result and shows it also holds for Algorithm 4.2. ${ }^{1}$

Theorem 4.2 The characterization of sets $C R D_{i}^{K}$ and $C_{i}^{K}\left(\lambda_{i}\right)$ in Algorithms 4.1 and 4.2 respectively, is independent of the order of elimination of choices.

${ }^{1}$ Note that the proof of Theorem 4.2 is not included in the appendix. Order independence for Algorithm 4.1 has been proven by Apt (2011). Moreover, proving order independence for Algorithm 4.2 is straightforward as the latter reduces to applying iterated elimination of strictly dominated choices to a set of modified games $\Gamma\left(c_{i}, \lambda_{i}\right)$ for each $c_{i} \in C_{i}$ and every player $i$. The order and speed independence of Algorithm 4.2 thus follows from that of iterated elimination of strictly dominated choices. 


\begin{tabular}{c|cccc}
\hline$\Gamma^{0}$ & $e$ & $f$ & $g$ & $h$ \\
\hline$a$ & 2,1 & 1,1 & 2,5 & 1,1 \\
$b$ & 5,4 & 1,0 & 2,1 & 3,0 \\
$c$ & 1,4 & 6,2 & 6,2 & 0,0 \\
$d$ & 2,3 & 2,5 & 0,2 & 0,3 \\
\hline
\end{tabular}

TABLE 4.7: Original Game $\Gamma^{0}$

\subsubsection{The Algorithm: An Example}

To illustrate the steps of Algorithm 4.1, consider the game $\Gamma^{0}$ in Table 4.7. Round 1 of the algorithm starts by eliminating choice $a$ of player 1 . Note that $a$ is weakly dominated by choice $b$ in game $\Gamma^{0}$ with $v_{1}\left(b, c_{2}\right)>v_{1}\left(a, c_{2}\right)$ for $c_{2} \in\{e, h\}$. Then $a$ must be weakly dominated by $b$ in every reduced game $\Gamma_{R}^{0}=\left(C_{1}, C_{2}^{\prime} ; v_{1}, v_{2}\right)$ with $C_{2}^{\prime} \cap\{e, h\} \neq \emptyset$. Similarly, $a$ is weakly dominated by choice $c$ in $\Gamma_{R}^{0}=\left(C_{1}, C_{2}^{*} ; v_{1}, v_{2}\right)$ for $C_{2}^{*} \in\{\{f, g\},\{f\},\{g\}\}$. Choice $a$ is thus weakly dominated in every possible reduced game $\Gamma_{R}^{0}=\left(C_{1}, C_{2}^{\prime} ; v_{1}, v_{2}\right)$.

In a similar manner, choice $h$ of player 2 is eliminated. Note that Choice $g$ weakly dominates $h$ in reduced games $\Gamma_{R}^{0}$ with $C_{1}^{\prime} \in\{\{a, b\},\{a, c\},\{b, c\}$ and $\{a\}$. Choice $f$ weakly dominates $h$ in reduced games $\Gamma_{R}^{0}$ with $C_{1}^{*} \in\{\{a, d\},\{c, d\}$ and $\{d\}\}$. Choice $e$ weakly dominates $h$ in all remaining reduced games.

Round 1 of the algorithm ends with the elimination of one choice for each player. The new modified game $\Gamma^{1}$ at the beginning of round 2 of the algorithm is shown in Table 4.8. In round 2, choice $g$ of player 2 is eliminated as it is weakly dominated in every reduced game $\Gamma_{R}^{1}$ by choice $e$. In other words, $g$ is strictly dominated by $e$, and so by Corollary 4.1 can never be RD-rational for any $\lambda_{2}$. For player 1, nothing is eliminated. The modified game $\Gamma^{2}$ at the beginning of round 3 of the algorithm is in Table 4.9.

\begin{tabular}{c|cccc}
\hline$\Gamma^{1}$ & $e$ & $f$ & $g$ & $h$ \\
\hline$a$ & & & & \\
$b$ & 5,4 & 1,0 & 2,1 & \\
$c$ & 1,4 & 6,2 & 6,2 & \\
$d$ & 2,3 & 2,5 & 0,2 & \\
\hline
\end{tabular}

TABle 4.8: Modified Game $\Gamma^{1}$

\begin{tabular}{c|cccc}
\hline$\Gamma^{2}$ & $e$ & $f$ & $g$ & $h$ \\
\hline$a$ & & & \\
$b$ & 5,4 & 1,0 & & \\
$c$ & 1,4 & 6,2 & & \\
$d$ & 2,3 & 2,5 & & \\
\hline
\end{tabular}

TABle 4.9: Modified Game $\Gamma^{2}$

Nothing is eliminated for either player in round 3, so the algorithm stops. Note that choice $d$ of player 1 is strictly dominated by a randomization between $b$ and $c$. However, it is not weakly dominated by any of the two and is therefore not eliminated. Sets $C R D_{1}^{K}=\{b, c, d\}$ and $C R D_{2}^{K}=\{e, f\}$. 
Now assume the value of each player's $\lambda_{i}$ has been common knowledge. Let $\lambda_{1}=\lambda_{2}=2$. By Theorem 4.2 and Lemma 4.3, applying Algorithm 4.2 to game $\Gamma^{0}$ (Table 4.7) yields the same final result as applying it to $\Gamma^{2}$ (Table 4.9). Starting from $\Gamma^{2}$, the first round of Algorithm 4.2 corresponds to the fourth round of elimination. For player 1 , choices $b$ and $c$ are optimal under classical expected utility maximization in $\Gamma^{2}$, i.e. for $\lambda_{1}=0$ for a belief assigning probability 1 to player 2's choices $e$ and $f$ respectively. Choices $b$ and $c$ are therefore also RD-optimal for the same beliefs and for $\lambda_{1}=2$, and are consequently not eliminated in round 4 .

Choice $d$ of player 1 is eliminated as it is strictly dominated in the decision problem $\Gamma_{1}(d, 2)$ (Table 4.10$)$ by a randomization $r_{1}(b)=0.55$ and $r_{1}(c)=0.45$. Round 5 of the algorithm starts with choices $b$ and $c$ for player 1 and choices $e$ and $f$ for player 2. It is clear that in the new reduced game, choice $f$ is strictly dominated by $e$, therefore never RD-optimal by Lemma 4.1 and so not RD-optimal for any belief for $\lambda_{1}=2$. Finally,

\begin{tabular}{c|cccc}
\hline$\Gamma^{3}(d, 2)$ & $e$ & $f$ & $g$ & $h$ \\
\hline$a$ & & & & \\
$b$ & 5 & -1 & & \\
$c$ & -1 & 6 & & \\
$d$ & 2 & 2 & & \\
\hline
\end{tabular}

TABle 4.10: Decision Problem $\Gamma^{3}(d, 2)$ choice $c$ of player 1 is eliminated in round 6, after which the algorithm stops. Choices surviving Algorithm 4.2 for $\lambda_{1}=\lambda_{2}=2$ are $C_{1}^{K}=\{b\}$ and $C_{2}^{K}=\{e\}$.

\subsection{The Algorithm as a Process of Deliberation}

While Theorem 4.1 proves that the algorithm exactly characterizes those choices that players can be expected to make under the epistemic concept of CBRDR, this section presents an alternative interpretation of the algorithm. Instead of it just being a computational tool, this section reinterprets Algorithm 4.1 as a model of reasoning. The algorithm is taken to represent the process of deliberation players go through to form their beliefs about their opponents' choices and beliefs and to eventually decide on their own choices. In this section we reconsider Algorithm 4.1 in the framework of Cubitt and Sugden (2011). A similar contribution within epistemic game theory in that regard is that of Perea (2015) where the algorithms characterizing the epistemic concepts of Common Belief in Future Rationality and Common Belief in Rationality are interpreted as reasoning procedures.

Players in our model are envisioned to go through three different phases from the moment they are first confronted with the game to the moment they make their choice. First, once each player $i$ is confronted with the game, he enters the deliberation phase where he form an expectation of his prospective choice and starts reasoning about his opponents in an attempt to distinguish those choices of the opponents that $i$ could deem possible and those that are impossible under 
a certain form of rationality and common belief therein. Once beliefs about the opponent are formed, player $i$ enters the implementation phase in which he reevaluates his choices taking his expected choice as well as his beliefs about his opponents as a reference point. Once choices have been reevaluated, player $i$ comes to phase 3 which entails making a choice.

Cubitt and Sugden (2011) introduce Categorization Procedures as a general framework in which the different iterations of procedures such as the one introduced in this paper can be interpreted as reasoning steps of players about their own choices as well as their opponents'. The specific form of rationality being modeled, in our case RD-rationality, is then a defining characteristic of the different reasoning steps players make. In what follows the definition of a categorization procedure as in Cubitt and Sugden (2011) is summarized. A categorization procedure corresponding to the algorithm of Iterated Elimination of RD-dominated Choices (IERDC) is then constructed. Using that procedure the reasoning of player $i$ in the deliberation phase is described first formally and then by means of an example. Finally, we address the question of whether our conclusions would still hold if the assumption of rational expectations is dropped.

\subsubsection{IERDC as a Categorization Procedure}

Let $C_{i}$ be the set of choices of player $i$ as defined throughout the paper. A categorization $<C_{i}^{+}, C_{i}^{-}>$of $C_{i}$ assigns player $i$ 's choices, whenever possible, into choices $C_{i}^{+}$that are always plausible for player $i$ and those $C_{i}^{-}$that are never plausible for $i$ under some specific form of rationality. A categorization $\left\langle C_{i}^{+}, C_{i}^{-}>\right.$of $C_{i}$ satisfies the properties: (i) $C_{i}^{+} \cap C_{i}^{-}=\emptyset$, (ii) $C_{i}^{-} \subset C_{i}$ and (iii) if $C_{i} \backslash C_{i}^{-}=\left\{c_{i}\right\}$, then $C_{i}^{+}=\left\{c_{i}\right\}$. A categorization procedure defines for every player $i$ a function $f_{i}: \Phi\left(C_{-i}\right) \rightarrow \Phi\left(C_{i}\right)$, where $\Phi\left(C_{i}\right)$ is the set of all categorizations of player $i$. Function $f_{i}$ has two main components; "An Allowability rule" and an "Assignment rule". The former specifies for player $i$ the set of allowable probability distributions over the opponents' choices; i.e. which choices of the opponents player $i$ can believe to occur with positive probability. The latter assigns each choice $c_{i} \in C_{i}$ to the appropriate category whenever possible.

The general definition of a categorization $<C_{i}^{+}, C_{i}^{-}>$includes the term $C_{i}^{+}$to allow for the possibility of a procedure that not only eliminates certain choices but also accumulates some other choices. In such case, each round classifies every player $i$ 's choices into one of three categories always optimal $C_{i}^{+}$, never optimal $C_{i}^{-}$and sometimes optimal $C_{i} \backslash\left(C_{i}^{+} \cup C_{i}^{-}\right)$. For the prupose of defining our procedure, $C_{i}^{+}=C R D_{i}^{1}$ and $C_{i}^{-}=D_{i}^{1}$ where $D_{i}^{1}=C_{i} \backslash C R D_{i}^{1}$. Note that in the case of IERDC, the crucial component of a categorization is the set $D_{i}^{1}$ as the procedure only eliminates choices in every round and does not further classify the remaining choices. 
The categorization procedure corresponding to IERDC starts for every player $i$ from the full set of his opponents' choice-combinations and defines the allowable set of beliefs $\Delta\left(C_{-i}\right)$ for player $i$ about his opponents' choice combinations, i.e. player $i$ starts off with deeming all subsets of choice-combinations of his opponents possible. The assignment rule then categorizes the set $C_{i}$ of every player $i$ into $\left\langle C R D_{i}^{1}, D_{i}^{1}>\right.$. In every round $k$, the allowability rule maps the set $C R D_{-i}^{k-1}$ into the set $\mathcal{P}^{*}\left(C R D_{-i}^{k-1}\right)=\mathcal{P}\left(C R D_{-i}^{k-1}\right) \backslash\{\emptyset\}$, where $\mathcal{P}\left(C_{-i}\right)$ is the power set of $C_{-i}$. The allowability rule thus requires in every round $k$, zero probability to be assigned to $c_{-i} \in D_{-i}^{k-1}$. The assignment rule in round $k$ then defines the set $D_{i}^{k}$ as the set of choices $c_{i} \in C R D_{i}^{k-1}$ of $i$ that are weakly dominated by some other choice $c_{i}^{\prime}$ on every $C_{-i}^{\prime} \in \mathcal{P}^{*}\left(C R D_{-i}^{k-1}\right)$, thereby also characterizing $C R D_{i}^{k}$. The procedure stops at $K$ where $D_{i}^{K}=D_{i}^{K+1}$ for all $i$.

\subsubsection{Reasoning Towards CBRDR}

The steps of Algorithm 4.1 formulated above into a categorization procedure will be used here to describe the reasoning process of each player $i$ in the deliberation phase. When first confronted with the game, player $i$ seeks to answer the following question: which choices can each opponent $j \in \mathcal{I} \backslash\{i\}$ be expected to play if he is RD-rational, believes his opponents are RD-rational, believes his opponents believe the same about him and so on?. Note that $\lambda_{j}$ is private information of each player $j$. For any player $j$, the set of subsets of opponents' choice-combinations $c_{-j}$ that $j$ could deem possible at the beginning of his deliberation process, is the set $\mathcal{P}^{*}\left(C_{-j}\right)$. Since $j$ is assumed to be RD-rational with some $\lambda_{j} \geq 0$, then by Lemma 4.1 player $j$ cannot RD-rationally choose any $c_{j}$ that is weakly dominated on ever $C_{-j}^{\prime} \in \mathcal{P}^{*}\left(C_{-j}\right)$ by some other $c_{j}^{\prime}$. Player $j$ can never be expected to play $c_{j} \in D_{j}^{1}$ regardless of his $\lambda_{j}$. Thus after the first round of deliberation, player $i$ will never be expected (or himself expect) to play $c_{i} \in D_{i}^{1}$ and will never assign any positive probability to $c_{-i} \in D_{-i}^{1}$.

In round $k$, player $i$ only deems choice-combinations $c_{-i} \in C R D_{-i}^{k-1}$ of his opponents possible. Since any RD-rational player $j$ never choose for $c_{j}$ that are weakly dominated by another choice $c_{j}^{\prime}$ on every subset of opponents' choice-combinations that $j$ deems possible, set $D_{j}^{k}$ includes those choices $c_{j}$ that are weakly dominated by some $c_{j}^{\prime}$ on every $C_{j}^{\prime} \in \mathcal{P}^{*}\left(C R D_{-j}^{k-1}\right)$. Thus after round $k$ of the deliberation process, player $i$ will never be expected (or himself expect) to play $c_{i} \in D_{i}^{k}$ and will never assign any positive probability to $c_{-i} \in D_{-i}^{k}$. Note that describing reasoning in this way is particularly appropriate because each player $i$ 's $\lambda_{i}$ is private information. Since the games we consider are finite, the deliberation procedure stops after a finite number of steps resulting in sets $\left(C R D_{1}^{K}, \ldots, C R D_{i}^{K}, \ldots, C R D_{n}^{K}\right)$ being formed. Every player $i$ only expects to play some $c_{i} \in C R D_{i}^{K}$ and believes each opponent $j$ of his will only choose from $C R D_{j}^{K}$. Once this conclusion is reached, players form their beliefs about their opponents' choices and their expectations about their own. 
To illustrate one deliberation step of the procedure, consider the game presented in section 4.4.1 and reproduced in Table 4.11 . Note that Table 4.11 only shows the payoffs of player 1 . For player 2 to determine which choices she can expect player 1 to rationally choose, player 2 considers all possible sets of choices $C_{2}^{\prime} \subseteq C_{2}$ of her own that player 1 may deem possible. No matter which

\begin{tabular}{c|cccc}
\hline$\Gamma^{0}$ & $e$ & $f$ & $g$ & $h$ \\
\hline$a$ & 2 & 1 & 2 & 1 \\
$b$ & 5 & 1 & 2 & 3 \\
$c$ & 1 & 6 & 6 & 0 \\
$d$ & 2 & 2 & 0 & 0 \\
\hline
\end{tabular}

TABLE 4.11: Game $\Gamma^{0}$ $C_{2}^{\prime}$ is considered, there is always a choice that weakly dominates $a$. For example, if player 1 were to assign positive probability only to player 2's choices $f$ and $g$, then choice $c$ is better than $a$ regardless of 1's actual loss aversion coefficient and regardless of the exact distribution of probability between $f$ and $g$. Choice $a$ is therefore deemed impossible for player 1 by player 2, i.e. player 2 will not assign any positive probability to the event of 1 playing $a$. Player 2 believes player 1 will reason about his opponent's choices in a similar manner. Furthermore, player 1 will never expect himself to choose $a$ regardless of the value of his loss aversion coefficient or his belief about his opponent as he knows that this would be an expectation he will not fulfill. Player 1 believes player 2 will reason about his own choices in the same way.

\subsubsection{How Do Players Form Expectations of their own Choices?}

Up till now, this section has assumed players form rational expectations about their own choices and carry through with their planned actions. During the deliberation process, each player $i$ was assumed to be trying to figure out which choices he "could expect" himself to carry out.This subsection explores whether our model could accommodate other forms of expectation formation about own actions. Many considerations could make some choice a candidate to be a player's reference choice, for example offering a guaranteed payoff regardless of what the opponents choose to do or having played that choice or a similar one in a context potentially similar but yet unrelated to the game under consideration. These are considerations that may cause player $i$ 's expectation of his own choice to divert away from the set of choices $C R D_{i}^{K}$ specified by our procedure.

Would such $c_{i}^{\prime \prime} \notin C R D_{i}^{K}$ when taken as a reference choice pass the implementation phase in which player $i$ reevaluates all his choices given his belief $b_{i}$, his expectation about his own choice and his $\lambda_{i}$ ? The answer to this question is no. Since such $b_{i} \in \triangle\left(C R D_{-i}^{K}\right)$, it is easy to see that if $c_{i}^{\prime \prime}$ was RD-optimal for that $b_{i}$ and for some $\lambda_{i}$, it would not have been eliminated in the first place. Note that the procedure is shown in Section 4.4 to be order insensitive, so any choice eliminated at some round $l$ can never become RD-rational in some round $k \geq l$. One could also envision altering the definition of RD-optimality of choices. For example, let $c_{i}^{*}$ be RD-optimal 
for some $b_{i}$ and for some $\lambda_{i}$ if $u_{i}\left(c_{i}^{*}, b_{i}, c_{i}^{\prime}, \lambda_{i}\right) \geq u_{i}\left(c_{i}, b_{i}, c_{i}^{\prime},, \lambda_{i}\right)$ for all $c_{i} \in C_{i}$ and where $c_{i}^{\prime} \neq c_{i}^{*}$ is a possibility. Proposition 4.1 implies that such alteration of the definition of RD-optimality will not affect the characterization of the final set of choices $C R D_{i}^{K}$.

Proposition 4.1 If $u_{i}\left(c_{i}^{*}, b_{i}, c_{i}^{\prime}, \lambda_{i}\right) \geq u_{i}\left(c_{i}, b_{i}, c_{i}^{\prime}, \lambda_{i}\right)$ for all $c_{i} \in C_{i}$, then it must be that $u_{i}\left(c_{i}^{*}, b_{i}, c_{i}^{*}, \lambda_{i}\right) \geq u_{i}\left(c_{i}, b_{i}, c_{i}^{*}, \lambda_{i}\right)$ for all $c_{i} \in C_{i}$.

Thus even if player $i$ would take as a reference choice some $c_{i}^{\prime \prime} \notin C R D_{i}^{K}$, he will eventually find it optimal to shift to some $c_{i} \in C R D_{i}^{K}$ at the implementation phase. Furthermore, should player $i$ form an expectation to play some $c_{i}^{\prime} \in C R D_{i}^{K}$ and switch to some other $c_{i}^{*}$ in the implementation stage, then it must be the case that $c_{i}^{*} \in C R D_{i}^{K}$.

\subsection{Discussion and Conclusion}

This section touches upon three extensions of the results of this paper. First, the case where gains as well as losses receive positive weights in the RD-utility function is considered. Second, the implications of the findings of this paper for the RD-utility model of KR06 are discussed. Third, it is shown that all these results can be easily extended to the case of multidimensional utility, i.e when the material utility of any given choice is composed of two or more distinct components.

\subsubsection{The Case of Gains Receiving Positive Weight}

Throughout the paper, the reference-dependent component of the utility function has assigned positive weight, represented by $\lambda_{i}$, only to losses. A more general form of our utility function is the following

$$
u_{i}^{\prime}\left(c_{i}, b_{i}, c_{i}^{*}\right):=\sum_{c_{-i} \in C_{-i}} b_{i}\left(c_{-i}\right)\left[v_{i}\left(c_{i}, c_{-i}\right)+\mu_{i}\left[v_{i}\left(c_{i}, c_{-i}\right)-v_{i}\left(c_{i}^{*}, c_{-i}\right)\right]\right]
$$

The term $\mu_{i}$ on the right hand side is then the gain-loss component of the utility function and satisfies the properties introduced by Kahneman and Tversky (1979) and formalized by Kőszegi and Rabin (2006). For every $c_{-i} \in C_{-i}$ function $\mu_{i}$ is defined as follows

$$
\mu_{i}\left(v_{i}\left(c_{i}, c_{-i}\right)-v_{i}\left(c_{i}^{*}, c_{-i}\right)\right)= \begin{cases}\eta_{i}\left(v_{i}\left(c_{i}, c_{-i}\right)-v_{i}\left(c_{i}^{*}, c_{-i}\right)\right) & \text { if } v_{i}\left(c_{i}, c_{-i}\right)-v_{i}\left(c_{i}^{*}, c_{-i}\right) \geq 0 \\ \eta_{i} \lambda_{i}\left(v_{i}\left(c_{i}, c_{-i}\right)-v_{i}\left(c_{i}^{*}, c_{-i}\right)\right) & \text { if } v_{i}\left(c_{i}, c_{-i}\right)-v_{i}\left(c_{i}^{*}, c_{-i}\right)<0\end{cases}
$$


Note that in this case $\lambda_{i} \geq 1$ is still the loss-aversion coefficient of player $i$ but now has a minimum value of 1 as losses are assumed to weigh more heavily than gains of the same magnitude. Furthermore, parameter $\eta_{i} \geq 0$ is the weight assigned to the gain-loss component of the RD-utility function. Proposition 4.1 shows that the characterization result of Lemma 4.3.1 extends to the above more general form of the utility function, at least as long as losses receive higher weights than gains of the same magnitude ${ }^{2}$.

Proposition 4.1 For any $c_{i}^{*}$ there exists some belief $b_{i} \in \Delta\left(C_{-i}\right)$, some $\eta_{i} \geq 0$ and some $\lambda_{i} \geq 1$ such that $u_{i}^{\prime}\left(c_{i}^{*}, b_{i}, c_{i}^{*}, \eta_{i}, \lambda_{i}\right) \geq u_{i}^{\prime}\left(c_{i}, b_{i}, c_{i}^{*}, \eta_{i}, \lambda_{i}\right)$ for all $c_{i} \in C_{i}$ if and only if there is at least one subset $C_{-i}^{\prime} \subseteq C_{-i}$ for which there exists no $c_{i} \in C_{i}$ that weakly dominates $c_{i}^{*}$ in the reduced game $\Gamma_{R}=\left(C_{i}, C_{-i}^{\prime} ; v_{i}, v_{-i}\right)$.

Consequently, the algorithm of Iterated Elimination of RD-dominated Choices also characterizes CBRDR where players preferences are represented by the modified linear RD-utility function defined above and where players' loss-aversion coefficients $\lambda_{i}$ and weight to the gain-loss component $\eta_{i}$ are private information. Thus the result of Theorem 4.1 would also hold for this modified RD-utility function.

\subsubsection{Links to the Kôszegi and Rabin (2006) Utility Function}

It is beyond this paper to completely characterize the set of choices that could be made under CBRDR and the utility function introduced by Köszegi and Rabin (2006). It is however easy to establish that the set of choices $C R D_{i}$ is always a superset of its counterpart under the Konszegi and Rabin (2006) utility function. Let the utility function $u_{i}^{K R}\left(c_{i}, b_{i}, c_{i}^{*}, \lambda_{i}\right)$ defined by the latter as follows

$$
u_{i}^{K R}\left(c_{i}, b_{i}, c_{i}^{*}, \lambda_{i}\right)=\sum_{c_{-i} \in C_{-i}} b_{i}\left(c_{-i}\right)\left[v_{i}\left(c_{i}, c_{-i}\right)-\sum_{c_{-i}^{\prime} \in C_{-i}} b_{i}\left(c_{-i}^{\prime}\right) \lambda_{i}\left[v_{i}\left(c_{i}^{*}, c_{-i}^{\prime}\right)-v_{i}\left(c_{i}, c_{-i}\right)\right]_{+}\right] .
$$

Choice $c_{i}^{*} \in C_{i}$ is RD-optimal for some $\lambda_{i} \geq 0$ and some $b_{i} \in \Delta\left(C_{-i}\right)$ if $u_{i}^{K R}\left(c_{i}^{*}, b_{i}, c_{i}^{*}, \lambda_{i}\right) \geq$ $u_{i}^{K R}\left(c_{i}, b_{i}, c_{i}^{*}, \lambda_{i}\right)$ for all $c_{i} \in C_{i}$. Lemma 4.2 shows that any choice $c_{i}^{*} \notin C R D_{i}$ of player $i$ cannot be RD-optimal for any $\lambda_{i}$ and any such $b_{i}$.

Proposition 4.2 For any choice $c_{i}^{*} \notin C R D_{i}$, there exists some $c_{i} \in C_{i}$ such that the following holds for any $\lambda_{i} \geq 0$ and any $b_{i} \in \triangle\left(C_{-i}\right)$

$$
u_{i}^{K R}\left(c_{i}, b_{i}, c_{i}^{*}, \lambda_{i}\right)>u_{i}^{K R}\left(c_{i}^{*}, b_{i}, c_{i}^{*}, \lambda_{i}\right)
$$

${ }^{2}$ Note that the proof of this proposition is not included in the Appendix as it involves only slight modifications to the proof of Lemma 4.1. The proof can be provided upon request though. 
While the above result doesn't characterize the set of choices that could be RD-optimal under the KR-utility function, it could help narrow down the set of choices to be examined in any given game.

\subsubsection{Multidimensional Utility}

Material utility $v_{i}\left(c_{i}, c_{-i}\right)$ is often considered in the literature to be the sum of the utility of an outcome $\left(c_{i}, c_{-i}\right)$ across multiple dimensions. This section allows for utility to be $m$-dimensional and separable across those dimensions and considers how such change would affect our results. Let

$$
v_{i}\left(c_{i}, c_{-i}\right)=\sum_{l=1}^{m} v_{i}^{l}\left(c_{i}, c_{-i}\right)
$$

The reference dependent utility function will then have the form

$$
u_{i}\left(c_{i}, b_{i}, c_{i}^{*}, \lambda_{i}\right):=\sum_{c_{-i} \in C_{-i}} b_{i}\left(c_{-i}\right) \sum_{l=1}^{m}\left[v_{i}^{l}\left(c_{i}, c_{-i}\right)-\lambda_{i}\left[v_{i}^{l}\left(c_{i}^{*}, c_{-i}\right)-v_{i}^{l}\left(c_{i}, c_{-i}\right)\right]_{+}\right]
$$

The the gain and loss component of the above function is again defined by

$$
\left[v_{i}^{l}\left(c_{i}^{*}, c_{-i}\right)-v_{i}^{l}\left(c_{i}, c_{-i}\right)\right]_{+}=\max \left\{\left(v_{i}^{l}\left(c_{i}^{*}, c_{-i}\right)-v_{i}^{l}\left(c_{i}, c_{-i}\right)\right), 0\right\}
$$

For multidimensional utilities, all our results would hold with an extra requirement. Lemma 4.1 would imply that a choice $c_{i}^{*} \notin C R D_{i}$ if and only if for every subset $C_{-i}^{\prime} \subseteq C_{-i}$ there exists some $c_{i}$ such that $v_{i}^{l}\left(c_{i}, c_{-i}\right) \geq v_{i}^{l}\left(c_{i}^{*}, c_{-i}\right)$ for all $c_{-i}$ and all $l$ and with $v_{i}^{l}\left(c_{i}, c_{-i}\right)>v_{i}^{l}\left(c_{i}^{*}, c_{-i}\right)$ for at least some $c_{-i}$ and some $l$. Corollary4.1 must also be modified accordingly. Furthermore, the algorithm could also be easily modified to accommodate the change ${ }^{3}$.

${ }^{3}$ Proofs of the results extended to the case of multidimensional utility are not included in the Appendix but can be provided upon request 


\subsection{Appendix}

\section{Proof of Lemma 4.1}

The proof of this lemma has two parts:

(A) For any choice $c_{i}^{*} \in C_{i}$ for which there exists some $c_{i} \in C_{i}$ for every subset $C_{-i}^{\prime} \subseteq C_{-i}$ such that $c_{i}^{*}$ is weakly dominated by $c_{i}$ in the reduced game $\Gamma_{R}=\left(C_{i}, C_{-i}^{\prime} ; v_{i}, v_{-i}\right)$, it must be that $c_{i}^{*} \notin C R D_{i}$.

(B) If choice $c_{i}^{*} \notin C R D_{i}$ then it must be that for every subset $C_{-i}^{\prime} \subseteq C_{-i}$ there is some choice $c_{i} \in C_{i}$ that weakly dominates $c_{i}^{*}$ in the reduced game $\Gamma_{R}=\left(C_{i}, C_{-i}^{\prime} ; v_{i}, v_{-i}\right)$.

Recall that the set $C R D_{i}$ of choices of player $i$ that are RD-optimal for some belief $b_{i} \in \triangle\left(C_{-i}\right)$ and some $\lambda_{i} \geq 0$ is defined as follows

$$
C R D_{i}=\left\{c_{i}^{*} \in C_{i} \mid \exists \lambda_{i} \geq 0 \text { and } b_{i} \in \Delta\left(C_{-i}\right) \text { s.t. } u_{i}\left(c_{i}^{*}, b_{i}, c_{i}^{*}, \lambda_{i}\right) \geq u_{i}\left(c_{i}, b_{i}, c_{i}^{*}, \lambda_{i}\right) \forall c_{i} \in C_{i}\right\}
$$

Also recall that

$$
u_{i}\left(c_{i}, b_{i}, c_{i}^{*}, \lambda_{i}\right)=\sum_{c_{-i} \in C_{-i}} b_{i}\left(c_{-i}\right)\left[v_{i}\left(c_{i}, c_{-i}\right)-\lambda_{i}\left[v_{i}\left(c_{i}^{*}, c_{-i}\right)-v_{i}\left(c_{i}, c_{-i}\right)\right]_{+}\right] .
$$

\section{Proof of Part A:}

Consider some $c_{i}^{*} \in C_{i}$ such that for every subset $C_{-i}^{\prime} \subseteq C_{-i}$, there exists some $c_{i} \in C_{i}$ such that $v_{i}\left(c_{i}, c_{-i}\right) \geq v_{i}\left(c_{i}^{*}, c_{-i}\right)$ for all $c_{-i} \in C_{-i}^{\prime}$ and $v_{i}\left(c_{i}, c_{-i}\right)>v_{i}\left(c_{i}^{*}, c_{-i}\right)$ for some $c_{-i} \in C_{-i}^{\prime}$. In what follows we show that for every belief $b_{i} \in \Delta\left(C_{-i}\right)$ and regardless of $\lambda_{i}$, there exists some $c_{i}$ with $u_{i}\left(c_{i}, b_{i}, c_{i}^{*}, \lambda_{i}\right)>u_{i}\left(c_{i}^{*}, b_{i}, c_{i}^{*}, \lambda_{i}\right)$.

Fix some $C_{-i}^{\prime} \subseteq C_{-i}$ and let $c_{i}^{\prime} \in C_{i}$ be the choice weakly dominating $c_{i}^{*}$ in $\Gamma_{R}=\left(C_{i}, C_{-i}^{\prime} ; v_{i}, v_{-i}\right)$. Consider some $b_{i} \in \Delta\left(C_{-i}^{\prime}\right)$ such that $b_{i}\left(c_{-i}\right)>0$ for all $c_{-i} \in C_{-i}^{\prime}$. Then by definition,

$$
u_{i}\left(c_{i}^{*}, b_{i}, c_{i}^{*}, \lambda_{i}\right)=\sum_{c_{-i} \in C_{-i}^{\prime}} b_{i}\left(c_{-i}\right) v_{i}\left(c_{i}^{*}, c_{-i}\right) .
$$

Since $c_{i}^{\prime}$ weakly dominates $c_{i}^{*}$ in $\Gamma_{R}=\left(C_{i}, C_{-i}^{\prime} ; v_{i}, v_{-i}\right)$, it follows that $\lambda_{i}\left[v_{i}\left(c_{i}^{*}, c_{-i}\right)-v_{i}\left(c_{i}^{\prime}, c_{-i}\right)\right]_{+}=$ 0 for all $c_{-i} \in C_{-i}^{\prime}$. Therefore,

$$
u_{i}\left(c_{i}^{\prime}, b_{i}, c_{i}^{*}, \lambda_{i}\right)=\sum_{c_{-i} \in C_{-i}^{\prime}} b_{i}\left(c_{-i}\right) v_{i}\left(c_{i}^{\prime}, c_{-i}\right)
$$


Note that in this case both $u_{i}\left(c_{i}^{*}, b_{i}, c_{i}^{*}, \lambda_{i}\right)$ and $u_{i}\left(c_{i}^{\prime}, b_{i}, c_{i}^{*}, \lambda_{i}\right)$ are independent of $\lambda_{i}$. Furthermore, by definition of $c_{i}^{\prime}$ and by construction of $b_{i} \in \Delta\left(C_{-i}^{\prime}\right)$, it follows that

$$
u_{i}\left(c_{i}^{\prime}, b_{i}, c_{i}^{*}, \lambda_{i}\right)>u_{i}\left(c_{i}^{*}, b_{i}, c_{i}^{*}, \lambda_{i}\right)
$$

Since the above holds for every $C_{-i}^{\prime} \subseteq C_{-i}$ by assumption, it follows that $c_{i}^{*}$ is never RD-optimal for a belief $b_{i} \in \Delta\left(C_{-i}^{\prime}\right)$ as constructed above, i.e. for any $b_{i} \in \Delta\left(C_{-i}\right)$. Moreover, because $u_{i}\left(c_{i}^{\prime}, b_{i}, c_{i}^{*}, \lambda_{i}\right)$ is always independent of $\lambda_{i}$, it follows that $c_{i}^{*}$ is never RD-optimal for any belief $b_{i}$ and for any $\lambda_{i} \geq 0$. Thus $c_{i}^{*} \notin C R D_{i}$.

\section{Proof of Part B:}

Consider some choice $c_{i}^{*} \notin C R D_{i}$. We prove by contradiction that there must be a choice $c_{i} \in C_{i}$ for every $C_{-i}^{\prime} \subseteq C_{-i}$ such that in game $\Gamma, v_{i}\left(c_{i}, c_{-i}\right) \geq v_{i}\left(c_{i}^{*}, c_{-i}\right)$ for all $c_{-i} \in C_{-i}^{\prime}$ and $v_{i}\left(c_{i}, c_{-i}\right)>v_{i}\left(c_{i}^{*}, c_{-i}\right)$ for at least some $c_{-i} \in C_{-i}^{\prime}$.

Fix some $c_{i}^{*} \notin C R D_{i}$. Assume there is some $C_{-i}^{*} \subseteq C_{-i}$ for which there exist no $c_{i} \in C_{i}$ weakly dominating $c_{i}^{*}$ in the reduced game $\Gamma_{R}=\left(C_{i}, C_{-i}^{*} ; v_{i}, v_{-i}\right)$. Consider an arbitrary belief $b_{i} \in \triangle\left(C_{-i}^{*}\right)$ where $b_{i}\left(c_{-i}\right)>0$ for all $c_{-i} \in C_{-i}^{*}$.

By assumption, there is no $c_{i} \in C_{i}$ weakly dominating $c_{i}^{*}$ in $\Gamma_{R}=\left(C_{i}, C_{-i}^{*} ; v_{i}, v_{-i}\right)$. In other words, each $c_{i} \in C_{i}$ is such that $v_{i}\left(c_{i}, c_{-i}\right) \geq v_{i}\left(c_{i}^{*}, c_{-i}\right)$ for some $c_{-i} \in C_{-i}^{*}$ and $v_{i}\left(c_{i}, c_{-i}\right) \leq v_{i}\left(c_{i}^{*}, c_{-i}\right)$ for some other $c_{-i} \in C_{-i}^{*}$. Without loss of generality, this step excludes the trivial case of a choice $c_{i}$ strictly dominated by $c_{i}^{*}$.

Define the sets $C_{-i}^{*+}=\left\{c_{-i} \in C_{-i}^{*} \mid v_{i}\left(c_{i}, c_{-i}\right) \geq v_{i}\left(c_{i}^{*}, c_{-i}\right)\right\}$ and $C_{-i}^{*-}=\left\{c_{-i} \in C_{-i}^{*} \mid v_{i}\left(c_{i}, c_{-i}\right)<\right.$ $\left.v_{i}\left(c_{i}^{*}, c_{-i}\right)\right\}$. Then for $c_{i}^{*}$,

$$
u_{i}\left(c_{i}^{*}, b_{i}, c_{i}^{*}, \lambda_{i}\right)=\sum_{c_{-i} \in C_{-i}^{*}} b_{i}\left(c_{-i}\right) v_{i}\left(c_{i}^{*}, c_{-i}\right),
$$

while for every $c_{i} \in C_{i} \backslash\left\{c_{i}^{*}\right\}$,

$$
u_{i}\left(c_{i}, b_{i}, c_{i}^{*}, \lambda_{i}\right)=\sum_{c_{-i} \in C_{-i}^{*}} b_{i}\left(c_{-i}\right) v_{i}\left(c_{i}, c_{-i}\right)-\lambda_{i} \sum_{c_{-i} \in C_{-i}^{*-}} b_{i}\left(c_{-i}\right)\left[v_{i}\left(c_{i}^{*}, c_{-i}\right)-v_{i}\left(c_{i}, c_{-i}\right)\right] .
$$

Note that $u_{i}\left(c_{i}^{*}, b_{i}, c_{i}^{*}, \lambda_{i}\right)$ is constant for all $\lambda_{i} \geq 0$ and so is the first term of $u_{i}\left(c_{i}, b_{i}, c_{i}^{*}, \lambda_{i}\right)$. The second term of $u_{i}\left(c_{i}, b_{i}, c_{i}^{*}, \lambda_{i}\right)$, however, is strictly negative by assumption and is a decreasing function of $\lambda_{i}$. Thus it is obvious that for any given $b_{i} \in \Delta\left(C_{-i}^{*}\right)$, there exists some $\lambda_{i}^{*}<\infty$ such that $u_{i}\left(c_{i}^{*}, b_{i}, c_{i}^{*}, \lambda_{i}^{*}\right) \geq u_{i}\left(c_{i}, b_{i}, c_{i}^{*}, \lambda_{i}^{*}\right)$ for all $c_{i} \in C_{i}$. Hence, $c_{i}^{*} \in C R D_{i}$. This creates a contradiction as $c_{i}^{*} \notin C R D_{i}$ by assumption. 


\section{Proof of Lemma 4.2}

Let $c_{i}^{*}$ be RD-optimal for some belief $b_{i} \in \Delta\left(C_{-i}\right)$ and some given $\lambda_{i}$. Thus for all $c_{i} \in C_{i}$,

$$
u_{i}\left(c_{i}^{*}, b_{i}, c_{i}^{*}, \lambda_{i}\right) \geq u_{i}\left(c_{i}, b_{i}, c_{i}^{*}, \lambda_{i}\right)
$$

Then, by Lemma 3 in Pearce (1984), this is equivalent to $c_{i}^{*}$ not being strictly dominated in the decision problem $\Gamma_{i}\left(c_{i}^{*}, \lambda_{i}\right)$ with the payoffs $u_{i}\left(c_{i}, c_{-i}\right)=v_{i}\left(c_{i}, c_{-i}\right)-\lambda_{i}\left[v_{i}\left(c_{i}^{*}, c_{-i}\right)-v_{i}\left(c_{i}, c_{-i}\right)\right]_{+}$. Thus $c_{i}^{*}$ is RD-optimal for some belief $b_{i}$ and given some $\lambda_{i} \geq 0$ if and only if there is no randomization $r_{i} \in \Delta\left(C_{i}\right)$ such that for every $c_{-i} \in C_{-i}$

$$
\sum_{c_{i} \in C_{i}} r_{i}\left(c_{i}\right)\left[v_{i}\left(c_{i}, c_{-i}\right)-\lambda_{i}\left[v_{i}\left(c_{i}^{*}, c_{-i}\right)-v_{i}\left(c_{i}, c_{-i}\right)\right]_{+}\right]>v_{i}\left(c_{i}^{*}, c_{-i}\right)
$$

\section{Proof of Theorem 4.1}

Let $M=\left(\hat{T}_{i}, \lambda_{i}, b_{i}\right)_{i \in \mathcal{I}}$ be some finite epistemic model. Let $\hat{T}_{i}^{1}$ be the set of types $t_{i}$ of player $i$ that express 1-fold belief in RD-rationality. Thus as in Definition 4.5 of section 4.2.2, $\hat{T}_{i}^{1}=\left\{t_{i} \in \hat{T}_{i}^{0} \mid b_{i}\left(t_{i}\right)\left(R D_{-i}^{0}\right)=1\right\}$, where for every $j \in \mathcal{I}$,

$$
R D_{j}^{0}:=\left\{\left(c_{j}^{*}, t_{j}\right) \in C_{j} \times \hat{T}_{j}^{0} \mid u_{j}\left(c_{j}^{*}, t_{j}, c_{j}^{*}\right) \geq u_{j}\left(c_{j}, t_{j}, c_{j}^{*}\right) \forall c_{j} \in C_{j}\right\} .
$$

Similarly, define for every player $j \in \mathcal{I}$ the set

$$
R D_{j}^{k-1}:=\left\{\left(c_{j}^{*}, t_{j}\right) \in C_{j} \times \hat{T}_{j}^{k-1} \mid u_{j}\left(c_{j}^{*}, t_{j}, c_{j}^{*}\right) \geq u_{j}\left(c_{j}, t_{j}, c_{j}^{*}\right) \quad \forall c_{j} \in C_{j}\right\},
$$

Define the set $\hat{T}_{i}^{k}$ of types $t_{i}$ of player $i$ expressing up-to $k$-fold belief in RD-rationality for $k \geq 2$ as follows

$$
\hat{T}_{i}^{k}=\left\{t_{i} \in \hat{T}_{i}^{k-1} \mid b_{i}\left(t_{i}\right)\left(R D_{-i}^{k-1}\right)=1\right\}
$$

Recall that $C R D_{i}^{k}$ is the set of choices of player $i$ surviving $k$ rounds of the Algorithm 4.1. Theorem 4.1 will be proven by induction on $k$ and involves proving the following two parts:

A) Any choice $c_{i}^{*}$ that is RD-optimal for some type $t_{i} \in \hat{T}_{i}^{k}$ must survive $k+1$ rounds of Algorithm 4.1.

B) Any choice $c_{i}^{*}$ that survives $k$ rounds of Algorithm 4.1 can be RD-optimal for some type $t_{i} \in \hat{T}_{i}^{k-1}$. 


\section{Proof of Part A:}

Induction Start: Let $k=1$ and let $c_{i}^{*}$ be RD-optimal for type $t_{i} \in \hat{T}_{i}^{1}$. Then $c_{i}^{*}$ is RD-optimal for the first order belief $\operatorname{marg}_{C_{-i}} b_{i}\left(t_{i}\right)$ of type $t_{i}$ and loss aversion coefficient $\lambda_{i}\left(t_{i}\right)$. Choice $c_{i}^{*}$ is thus RD-optimal for some belief $b_{i} \in \Delta\left(C_{-i}\right)$ and some $\lambda_{i} \geq 0$. Hence, $c_{i}^{*} \in C R D_{i}^{1}$. Furthermore, note that $t_{i} \in \hat{T}_{i}^{1}$, thus $b_{i}\left(t_{i}\right)\left(R D_{-i}^{0}\right)=1$. Note that for every $j \neq i$, any choice $c_{j}^{*}$ with $\left(c_{j}^{*}, t_{j}\right) \in R D_{j}^{0}$ is RD-optimal for $b_{j}\left(t_{j}\right)$ and $\lambda_{j}\left(t_{j}\right)$. It follows that such $c_{j}^{*} \in C R D_{j}^{1}$. Choice $c_{i}^{*}$ being RD-optimal for $t_{i} \in \hat{T}_{i}^{1}$ is thus RD-optimal for some belief $b_{i} \in \triangle\left(C R D_{-i}^{1}\right)$. Thus $c_{i}^{*} \in C R D_{i}^{2}$.

Induction Assumption: Assume that any choice $c_{i}$ that is RD-optimal for some type $t_{i} \in \hat{T}_{i}^{k-1}$ must survive $k$ rounds of Algorithm 4.1.

Induction Step: Let $k \geq 2$ and show that whenever $c_{i}^{*}$ is RD-optimal for some $t_{i} \in \hat{T}_{i}^{k}$, it must be that $c_{i}^{*} \in C R D_{i}^{k+1}$. Let $c_{i}^{*}$ be RD-optimal for type $t_{i} \in \hat{T}_{i}^{k}$. Since any $\hat{T}_{i}^{k} \subseteq \hat{T}_{i}^{k-1}$, it follows by the induction assumption that $c_{i}^{*} \in C R D_{i}^{k}$.

By definition, $b_{i}\left(t_{i}\right)\left(R D_{-i}^{k-1}\right)=1$ for any $t_{i} \in \hat{T}_{i}^{k}$. Moreover, by the induction assumption, $c_{-i} \in C R D_{-i}^{k}$ whenever there is some $t_{-i}$ such that $\left(c_{-i}, t_{-i}\right) \in R D_{-i}^{k-1}$. Thus any choice $c_{i}^{*}$ that is RD-optimal for some $t_{i} \in \hat{T}_{i}^{k}$, is RD-optimal for some belief $\operatorname{marg}_{C_{-i}} b_{i}\left(t_{i}\right) \in \triangle\left(C R D_{-i}^{k}\right)$ and some $\lambda_{i}\left(t_{i}\right)$. Thus $c_{i}^{*} \in C R D_{i}^{k+1}$.

\section{Proof of Part B:}

The proof of this part is structured as follows: In step 0 we show that any choice $c_{i} \in C R D_{i}^{k}$ that is by definition RD-optimal in the modified game $\Gamma^{k-1}$ for some belief $b_{i} \in \triangle\left(C R D_{-i}^{k-1}\right)$ and some $\lambda_{i} \geq 0$, must also be RD-optimal for the same belief and the same $\lambda_{i} \geq 0$ in the original game $\Gamma$. Step 0 is a result that will be used in steps 1 and 2. Step 1 defines for every choice $c_{i} \in C R D_{i}^{k}$ for some $k \geq 0$ a belief $b_{i}^{c_{i}}$ and a loss aversion coefficient $\lambda^{c_{i}}$ for which $c_{i}$ is RD-optimal. Step 2 constructs for every such choice a corresponding type $t_{i}^{c_{i}}$ and defines the finite epistemic model $M$. Step 3 proves that every type $t_{i}^{c_{i}}$ for $c_{i} \in C R D_{i}^{k}$ with $k<K$ expresses up to $(k-1)$-fold belief in RD-rationality. Step 4 shows that every type $t_{i}^{c_{i}}$ for $c_{i} \in C R D_{i}^{K}$ expresses common belief in RD-rationality.

\section{Step 0: RD-Optimality of a Choice in $\Gamma^{\mathrm{k}-1}$ implies its RD-optimality in $\Gamma$ :}

Take some $c_{i} \in C R D_{i}^{k}$. Then $c_{i}$ is by definition RD-optimal in the modified game $\Gamma^{k-1}$ for some belief $b_{i} \in \triangle\left(C R D_{-i}^{k-1}\right)$ and some $\lambda_{i} \geq 0$. We prove by contradiction that this implies that $c_{i}$ must also be RD-optimal in $\Gamma$. Assume that there is a choice $c_{i}^{\prime}$ in $\Gamma$ and not in $\Gamma^{k-1}$ such that for all $c_{i} \in C_{i}$

$$
u_{i}\left(c_{i}^{\prime}, b_{i}, c_{i}, \lambda_{i}\right)>u_{i}\left(c_{i}, b_{i}, c_{i}, \lambda_{i}\right)
$$


By definition of $u_{i}\left(c_{i}^{\prime}, b_{i}, c_{i}, \lambda_{i}\right)$, the following holds $u_{i}\left(c_{i}^{\prime}, b_{i}, c_{i}^{\prime}, \lambda_{i}\right) \geq u_{i}\left(c_{i}^{\prime}, b_{i}, c_{i}, \lambda_{i}\right)$ and $u_{i}\left(c_{i}, b_{i}, c_{i}, \lambda_{i}\right) \geq u_{i}\left(c_{i}, b_{i}, c_{i}^{\prime}, \lambda_{i}\right)$. It follows that in $\Gamma$,

$$
u_{i}\left(c_{i}^{\prime}, b_{i}, c_{i}^{\prime}, \lambda_{i}\right)>u_{i}\left(c_{i}, b_{i}, c_{i}^{\prime}, \lambda_{i}\right)
$$

Thus $c_{i}^{\prime}$ is RD-optimal for belief $b_{i} \in \triangle\left(C R D_{-i}^{k-1}\right)$ and $\lambda_{i}$ in $\Gamma$ and so should have also been in $\Gamma^{k-1}$. Thus it must be that $c_{i}^{\prime} \in C R D_{i}^{k}$ which creates a contradiction. Hence, any choice $c_{i} \in C R D_{i}^{k}$ is also RD-optimal for some $b_{i} \in \triangle\left(C R D_{-i}^{k-1}\right)$ and $\lambda_{i}$ in $\Gamma$.

\section{Step 1: Belief Construction:}

Define for each choice $c_{i} \in C R D_{i}^{K}$ a belief $b_{i}^{c_{i}} \in \Delta\left(C R D_{-i}^{K}\right)$ and a loss aversion coefficient $\lambda^{c_{i}}$ such that $u_{i}\left(c_{i}, b_{i}, c_{i}, \lambda^{c_{i}}\right) \geq u_{i}\left(c_{i}^{\prime}, b_{i}, c_{i}, \lambda^{c_{i}}\right)$ for all $c_{i}^{\prime} \in C_{i}$. Thus $b_{i}^{c_{i}}$ and $\lambda^{c_{i}}$ are such that $c_{i}$ is RD-optimal in the original game $\Gamma$, which is possible by step 0 above. For every $0 \leq k<K$, define for each $c_{i} \in C R D_{i}^{k} \backslash C R D_{i}^{k+1}$ a belief $b_{i}^{c_{i}} \in \triangle\left(C R D_{-i}^{k-1}\right)$ and some $\lambda^{c_{i}}$ such that $c_{i}$ is RD-optimal in $\Gamma$.

\section{Step 2: Type Construction:}

Using the beliefs and loss aversion coefficients from step 1, this step constructs for every choice $c_{i}$ of player $i$ an epistemic type $t_{i}^{c_{i}}$ for which $c_{i}$ is RD-optimal. Let $T_{i}$ be the finite set of types of player $i$ defined as $T_{i}=\left\{t_{i}^{c_{i}} \mid c_{i} \in C R D_{i}^{1}\right\}$. For every $1<k \leq K$, let $T_{i}^{k}=\left\{t_{i}^{c_{i}} \mid c_{i} \in C R D_{i}^{k}\right\}$. By definition of $C R D_{i}^{k}$, it follows that $T_{i}^{K} \subseteq T_{i}^{K-1} \subseteq \ldots . \subseteq T_{i}^{k} \ldots . \subseteq T_{i}$. In what follows the beliefs and loss aversion coefficients of the constructed types are defined.

For every $c_{i} \in C_{i}^{K}$ and for every opponents' choice combination $\left(c_{1}, \ldots, c_{i-1}, c_{i+1}, \ldots c_{n}\right)$ of player $i$, let

$$
b_{i}\left(t_{i}^{c_{i}}\right)\left(\left(c_{1}, t_{1}^{c_{1}}\right), \ldots .\left(c_{i-1}, t_{i-1}^{c_{i-1}}\right),\left(c_{i+1}, t_{i+1}^{c_{i+1}}\right), \ldots . .\left(c_{n}, t_{n}^{c_{n}}\right)\right)=b_{i}^{c_{i}}\left(c_{1}, \ldots, c_{i-1}, c_{i+1}, \ldots c_{n}\right) .
$$

Furthermore, let $\lambda_{i}\left(t_{i}^{c_{i}}\right)=\lambda^{c_{i}}$. Since $b_{i}^{c_{i}} \in \Delta\left(C R D_{-i}^{K}\right)$ and since $t_{i}^{c_{i}}$ assigns positive probability only to opponents' choice-type combinations $\left(c_{j}, t_{j}\right)$ where $t_{j}=t_{j}^{c_{j}}$, then $t_{i}^{c_{i}}$ assigns positive probability only to opponents' type combinations $t_{-i} \in T_{i}^{K}$.

In a similar manner, let $b_{i}\left(t_{i}^{c_{i}}\right)$ for every $c_{i} \in C R D_{i}^{k} \backslash C R D_{i}^{k+1}$ and $1<k<K$ be such that,

$$
b_{i}\left(t_{i}^{c_{i}}\right)\left(\left(c_{1}, t_{1}^{c_{1}}\right), \ldots .\left(c_{i-1}, t_{i-1}^{c_{i-1}}\right),\left(c_{i+1}, t_{i+1}^{c_{i+1}}\right), \ldots . .\left(c_{n}, t_{n}^{c_{n}}\right)\right)=b_{i}^{c_{i}}\left(c_{1}, \ldots, c_{i-1}, c_{i+1}, \ldots c_{n}\right),
$$


for every choice combination $c_{-i}$ of the opponents. Define the loss aversion coefficient of every such type by $\lambda_{i}\left(t_{i}^{c_{i}}\right)=\lambda^{c_{i}}$. Note that such type $t_{i}^{c_{i}} \in T_{i}^{k} \backslash T_{i}^{k+1}$. Since $b_{i}^{c_{i}} \in \triangle\left(C R D_{-i}^{k-1}\right)$ and by construction of $b_{i}\left(t_{i}^{c_{i}}\right)$, it follows that $t_{i}^{c_{i}}$ assigns positive probability only to $t_{-i} \in T_{-i}^{k-1}$.

Finally, for any $t_{i}^{c_{i}}$ with $c_{i} \in C R D_{i}^{1} \backslash C R D_{i}^{2}$ let $b_{i}\left(t_{i}^{c_{i}}\right)$ be such that for each combination $c_{-i}$, $b_{i}\left(t_{i}^{c_{i}}\right)\left(\left(c_{1}, t_{1}\right), \ldots .\left(c_{i-1}, t_{i-1}\right),\left(c_{i+1}, t_{i+1}\right), \ldots . .\left(c_{n}, t_{n}\right)\right)=b_{i}^{c_{i}}\left(c_{1}, \ldots, c_{i-1}, c_{i+1}, \ldots c_{n}\right)$, where $t_{j} \in T_{j}$ is an arbitrary type of player $j \neq i$. Moreover, let $\lambda_{i}\left(t_{i}^{c_{i}}\right)=\lambda^{c_{i}}$.

\section{Step 3: Type in $\mathrm{T}_{\mathrm{i}}^{\mathrm{k}}$ express up to ( $\left.\mathrm{k}-1\right)$-fold Belief in RD-Rationality:}

Induction Start: Let $k=2$. Take some $c_{i} \in C R D_{i}^{2}$. By the construction of types in step 2, it is clear that type $t_{i}^{c_{i}}$ assigns positive probability only to opponents' choice-type combinations $\left(c_{j}, t_{j}^{c_{j}}\right)$ where for every $j \neq i$ choice $c_{j}$ is RD-optimal for $t_{j}^{c_{j}}$. Thus player $i$ 's types in $T_{i}^{2}$ express 1 -fold belief in RD-rationality.

Induction Assumption: Assume that for every player $i$ any epistemic type $t_{i} \in T_{i}^{k-1}$ expresses up to $(k-2)$-fold belief in RD-rationality.

Induction Step: Let $k \geq 3$ and consider some $t_{i}^{c_{i}} \in T_{i}^{k}$ for some $c_{i} \in C R D_{i}^{k}$. By construction of types in step $2, t_{i}^{c_{i}}$ assigns positive probability only to opponents' types $t_{j} \in T_{j}^{k-1}$ for every $j \neq i$, which by induction assumption express up to $(k-2)$-fold belief in RD-rationality. Every type $t_{i}^{c_{i}} \in T_{i}^{k}$, thus expresses up to $(k-1)$-fold belief in RD-rationality.

By construction of beliefs and types in steps 1 and 2, every choice $c_{i} \in C R D_{i}^{k}$ is RD-optimal for some type $t_{i}^{c_{i}} \in T_{i}^{k}$. Thus every choice $c_{i} \in C R D_{i}^{k}$ is RD-optimal for some type expressing up to $k-1$ belief in RD-rationality.

\section{Step 4: Type in $\mathrm{T}_{\mathrm{i}}^{\mathrm{K}}$ express Common Belief in RD-Rationality:}

From step 3, it has been shown that every $t_{i} \in T_{i}^{K}$ expresses up to $(K-1)$-fold belief in RDrationality. Now consider $k \geq K$. Note that every $t_{i} \in T_{i}^{K}$ assigns positive probability only to opponents' types $t_{j} \in T_{j}^{K}$. By step 3 above, every $t_{i} \in T_{i}^{K}$ expresses up to $K$-fold belief in RD-rationality. By induction on $k$, it is easy to see that any $t_{i} \in T_{i}^{K}$ expresses common belief in RD-rationality. By construction of beliefs and types in steps 1 and 2, every choice $c_{i} \in C R D_{i}^{K}$ is RD-optimal for some type $t_{i}^{c_{i}} \in T_{i}^{K}$. Thus every choice $c_{i} \in C R D_{i}^{K}$ is RD-optimal for some type expressing common belief in RD-rationality.

\section{Proof of Lemma 4.3}

Fix some $\lambda_{i}^{*}$ and consider a choice $c_{i} \in C_{i}^{1}\left(\lambda_{i}^{*}\right)$. Since $c_{i}$ is RD-optimal for some belief $b_{i} \in$ $\triangle\left(C_{-i}\right)$ and for the given $\lambda_{i}^{*}$, it must be that $c_{i} \in C R D_{i}^{1}$. Assume that whenever $c_{i} \in C_{i}^{k-1}\left(\lambda_{i}^{*}\right)$ it 
is also the case that $c_{i} \in C R D_{i}^{k-1}$. Then we prove by induction on $k$ that any $c_{i} \in C_{i}^{k}\left(\lambda_{i}^{*}\right)$ must also be $c_{i} \in C R D_{i}^{k}$.

Let $k \geq 2$ and take some $c_{i} \in C_{i}^{k}\left(\lambda_{i}^{*}\right)$. Then $c_{i}$ must be RD-optimal for some $b_{i} \in \Delta\left(C_{-i}^{k-1}\left(\lambda_{-i}^{*}\right)\right)$ and for the given $\lambda_{i}^{*}$. From the induction assumption, $C_{j}^{k-1}\left(\lambda_{j}^{*}\right) \subseteq C R D_{j}^{k-1}$ for all $j$. Hence, by Step 0 in the proof of part B of theorem 4.1, it follows that $u_{i}\left(c_{i}, b_{i}, c_{i}, \lambda_{i}^{*}\right) \geq u_{i}\left(c_{i}^{\prime}, b_{i}, c_{i}, \lambda_{i}^{*}\right)$ for all $c_{i}^{\prime} \in C R D_{i}^{k}$, for some $b_{i} \in \triangle\left(C R D_{-i}^{k-1}\right)$ and given $\lambda_{i}^{*}$.

*Proof of Proposition 4.1 Take some choice $c_{i}^{*}$ such that $u_{i}\left(c_{i}^{*}, b_{i}, c_{i}^{\prime}, \lambda_{i}\right) \geq u_{i}\left(c_{i}, b_{i}, c_{i}^{\prime}, \lambda_{i}\right)$ for all $c_{i} \in C_{i}$ for some reference choice $c_{i}^{\prime} \neq c_{i}^{*}$. Then for all $c_{i} \in C_{i}$,

$$
\begin{gathered}
\sum_{c_{-i} \in C_{-i}} b_{i}\left(c_{-i}\right)\left[v_{i}\left(c_{i}^{*}, c_{-i}\right)-\lambda_{i}\left[v_{i}\left(c_{i}^{\prime}, c_{-i}\right)-v_{i}\left(c_{i}^{*}, c_{-i}\right)\right]_{+}\right] \geq \\
\sum_{c_{-i} \in C_{-i}} b_{i}\left(c_{-i}\right)\left[v_{i}\left(c_{i}, c_{-i}\right)-\lambda_{i}\left[v_{i}\left(c_{i}^{\prime}, c_{-i}\right)-v_{i}\left(c_{i}, c_{-i}\right)\right]_{+}\right] .
\end{gathered}
$$

To simplify notation, let $E U\left(c_{i}, b_{i}\right)=\sum_{c_{-i}} b_{i}\left(c_{-i}\right) v_{i}\left(c_{i}, c_{-i}\right)$.

The above implies:

(1) $E U\left(c_{i}^{*}, b_{i}\right)-E U\left(c_{i}^{\prime}, b_{i}\right) \geq \sum_{c_{-i} \in C_{-i}} b_{i}\left(c_{-i}\right) \lambda_{i}\left[v_{i}\left(c_{i}^{\prime}, c_{-i}\right)-v_{i}\left(c_{i}^{*}, c_{-i}\right)\right]_{+}$

(2) $E U\left(c_{i}^{*}, b_{i}\right)-E U\left(c_{i}, b_{i}\right) \geq \sum_{c_{-i} \in C_{-i}} b_{i}\left(c_{-i}\right) \lambda_{i}\left[\left[v_{i}\left(c_{i}^{\prime}, c_{-i}\right)-v_{i}\left(c_{i}^{*}, c_{-i}\right)\right]_{+}-\left[v_{i}\left(c_{i}^{\prime}, c_{-i}\right)-v_{i}\left(c_{i}, c_{-i}\right)\right]_{+}\right]$

The proof of the lemma reduces to proving that satisfying (1) and (2) implies the following holds for every $c_{i} \in C_{i}$

$$
E U\left(c_{i}^{*}, b_{i}\right)-E U\left(c_{i}, b_{i}\right) \geq-\sum_{c_{-i} \in C_{-i}} b_{i}\left(c_{-i}\right) \lambda_{i}\left[v_{i}\left(c_{i}^{*}, c_{-i}\right)-v_{i}\left(c_{i}, c_{-i}\right)\right]_{+}
$$

Fix some $c_{-i} \in C_{-i}$, then

$$
\begin{aligned}
\lambda_{i}\left[v_{i}\left(c_{i}^{*}, c_{-i}\right)-v_{i}\left(c_{i}, c_{-i}\right)\right]_{+} & =\lambda_{i}\left[v_{i}\left(c_{i}^{*}, c_{-i}\right)-v_{i}\left(c_{i}^{\prime}, c_{-i}\right)+v_{i}\left(c_{i}^{\prime}, c_{-i}\right)-v_{i}\left(c_{i}, c_{-i}\right)\right]_{+} \\
& =\lambda_{i}\left[\left[v_{i}\left(c_{i}^{\prime}, c_{-i}\right)-v_{i}\left(c_{i}, c_{-i}\right)\right]-\left[v_{i}\left(c_{i}^{\prime}, c_{-i}\right)-v_{i}\left(c_{i}^{*}, c_{-i}\right)\right]\right]_{+} \\
& \geq \lambda_{i}\left[v_{i}\left(c_{i}^{\prime}, c_{-i}\right)-v_{i}\left(c_{i}, c_{-i}\right)\right]_{+}-\lambda_{i}\left[v_{i}\left(c_{i}^{\prime}, c_{-i}\right)-v_{i}\left(c_{i}^{*}, c_{-i}\right)\right]_{+}
\end{aligned}
$$

Summing over all $c_{-i} \in C_{-i}$, 


$$
\begin{aligned}
& \sum_{c_{-i} \in C_{-i}} b_{i}\left(c_{-i}\right) \lambda_{i}\left[v_{i}\left(c_{i}^{*}, c_{-i}\right)-v_{i}\left(c_{i}, c_{-i}\right)\right]_{+} \geq \\
& \sum_{c_{-i} \in C_{-i}} b_{i}\left(c_{-i}\right)\left[\lambda_{i}\left[v_{i}\left(c_{i}^{\prime}, c_{-i}\right)-v_{i}\left(c_{i}, c_{-i}\right)\right]_{+}-\lambda_{i}\left[v_{i}\left(c_{i}^{\prime}, c_{-i}\right)-v_{i}\left(c_{i}^{*}, c_{-i}\right)\right]_{+}\right]
\end{aligned}
$$

Or,

$$
\begin{aligned}
& -\sum_{c_{-i} \in C_{-i}} b_{i}\left(c_{-i}\right) \lambda_{i}\left[v_{i}\left(c_{i}^{*}, c_{-i}\right)-v_{i}\left(c_{i}, c_{-i}\right)\right]_{+} \leq \\
& \sum_{c_{-i} \in C_{-i}} b_{i}\left(c_{-i}\right)\left[\lambda_{i}\left[v_{i}\left(c_{i}^{\prime}, c_{-i}\right)-v_{i}\left(c_{i}^{*}, c_{-i}\right)\right]_{+}-\lambda_{i}\left[v_{i}\left(c_{i}^{\prime}, c_{-i}\right)-v_{i}\left(c_{i}, c_{-i}\right)\right]_{+}\right]
\end{aligned}
$$

From (2), $E U\left(c_{i}^{*}, b_{i}\right)-E U\left(c_{i}, b_{i}\right)$ is greater than or equal the RHS of (4) and so is also greater than or equal the LHS of (4). This proves that if (2) holds for every $c_{i}$ then so does (3).

\section{Proof of Proposition 4.2}

Consider some $c_{i}^{*} \notin C R D_{i}$ and fix any $C_{-i}^{\prime} \subseteq C_{-i}$. Since $c_{i}^{*} \notin C R D_{i}$ there must be a choice $c_{i} \in C_{i}$ weakly dominating $c_{i}^{*}$ in the reduced game $\Gamma=\left(C_{i}, C_{-i}^{\prime} ; v_{i}, v_{-i}\right)$ for every $C_{-i}^{\prime}$. In what follows we show that for such choice $c_{i}$,

$$
u_{i}^{K}\left(c_{i}, b_{i}, c_{i}^{*}, \lambda_{i}\right)>u_{i}^{K}\left(c_{i}^{*}, b_{i}, c_{i}^{*}, \lambda_{i}\right)
$$

for any $\lambda_{i} \geq 0$ and any $b_{i} \in \Delta\left(C_{-i}^{\prime}\right)$ with $b_{i}\left(c_{-i}\right)>0$ for all $c_{-i} \in C_{-i}^{\prime}$. Recall that

$$
u_{i}^{K}\left(c_{i}, b_{i}, c_{i}^{*}, \lambda_{i}\right)=\sum_{c_{-i} \in C_{-i}} b_{i}\left(c_{-i}\right)\left[v_{i}\left(c_{i}, c_{-i}\right)-\sum_{c_{-i}^{\prime} \in C_{-i}} b_{i}\left(c_{-i}^{\prime}\right) \lambda_{i}\left[v_{i}\left(c_{i}^{*}, c_{-i}^{\prime}\right)-v_{i}\left(c_{i}, c_{-i}\right)\right]_{+}\right]
$$

and

$$
u_{i}^{K}\left(c_{i}^{*}, b_{i}, c_{i}^{*}, \lambda_{i}\right)=\sum_{c_{-i} \in C_{-i}} b_{i}\left(c_{-i}\right)\left[v_{i}\left(c_{i}^{*}, c_{-i}\right)-\sum_{c_{-i}^{\prime} \in C_{-i}} b_{i}\left(c_{-i}^{\prime}\right) \lambda_{i}\left[v_{i}\left(c_{i}^{*}, c_{-i}^{\prime}\right)-v_{i}\left(c_{i}^{*}, c_{-i}\right)\right]_{+}\right] .
$$

Since $c_{i}$ weakly dominates $c_{i}^{*}$ in $\Gamma=\left(C_{i}, C_{-i}^{\prime} ; v_{i}, v_{-i}\right)$, then by definition of $b_{i} \in \Delta\left(C_{-i}^{\prime}\right)$,

$$
\sum_{c_{-i} \in C_{-i}^{\prime}} b_{i}\left(c_{-i}\right) v_{i}\left(c_{i}, c_{-i}\right)>\sum_{c_{-i} \in C_{-i}^{\prime}} b_{i}\left(c_{-i}\right) v_{i}\left(c_{i}^{*}, c_{-i}\right) \text {. }
$$

It suffices to show that for every $c_{-i} \in C_{-i}^{\prime}$,

$$
\sum_{c_{-i}^{\prime} \in C_{-i}^{\prime}} b_{i}\left(c_{-i}^{\prime}\right)\left[\lambda_{i}\left[v_{i}\left(c_{i}^{*}, c_{-i}^{\prime}\right)-v_{i}\left(c_{i}, c_{-i}\right)\right]_{+}-\lambda_{i}\left[v_{i}\left(c_{i}^{*}, c_{-i}^{\prime}\right)-v_{i}\left(c_{i}^{*}, c_{-i}\right)\right]_{+}\right] \leq 0
$$


Note that for any $c_{-i}^{\prime} \in C_{-i}$,

$$
\lambda_{i}\left[v_{i}\left(c_{i}^{*}, c_{-i}^{\prime}\right)-v_{i}\left(c_{i}, c_{-i}\right)\right]_{+}-\lambda_{i}\left[v_{i}\left(c_{i}^{*}, c_{-i}^{\prime}\right)-v_{i}\left(c_{i}^{*}, c_{-i}\right)\right]_{+} \leq \lambda_{i}\left[v_{i}\left(c_{i}^{*}, c_{-i}\right)-v_{i}\left(c_{i}, c_{-i}\right)\right]_{+} \cdot
$$

Since $v_{i}\left(c_{i}, c_{-i}\right) \geq v_{i}\left(c_{i}^{*}, c_{-i}\right)$ for any $c_{-i} \in C_{-i}^{\prime}$ and since $b_{i}\left(c_{-i}\right)>0$ if and only if $c_{-i} \in C_{-i}^{\prime}$,

$$
\sum_{c_{-i}^{\prime} \in C_{-i}^{\prime}} b_{i}\left(c_{-i}^{\prime}\right) \lambda_{i}\left[v_{i}\left(c_{i}^{*}, c_{-i}\right)-v_{i}\left(c_{i}, c_{-i}\right)\right]_{+}=0
$$

It follows that for every $c_{-i} \in C_{-i}^{\prime}$,

$$
\sum_{c_{-i}^{\prime} \in C_{-i}^{\prime}} b_{i}\left(c_{-i}^{\prime}\right)\left[\lambda_{i}\left[v_{i}\left(c_{i}^{*}, c_{-i}^{\prime}\right)-v_{i}\left(c_{i}, c_{-i}\right)\right]_{+}-\lambda_{i}\left[v_{i}\left(c_{i}^{*}, c_{-i}^{\prime}\right)-v_{i}\left(c_{i}^{*}, c_{-i}\right)\right]_{+}\right] \leq 0 .
$$




\section{Bibliography}

Aliprantis C and Border K (1999). Infinite dimensional analysis. Springer.

Apt K.R (2011). Direct Proofs of Order Independence.Economics Bulletin 31(1): 106-115.

Aumann R (1976). Agreeing to disagree. Ann. Stat. 4: 1236-1239.

Bach C, Perea A (2014). Utility Proportional Beliefs. International Journal of Game Theory 43: 881-902.

Basu K (1994). The Traveler's Dilemma: Paradoxes of Rationality in Game Theory. The American Economic Review 84(2): 391-394.

Battigalli P (1997). On Rationalizability in Extensive Games. Journal of Economic Theory 74: $40-61$.

Battigalli, P, Siniscalchi M (2002). Strong belief and forward induction reasoning. Journal of Economic Theory 106: 356-391.

Battigalli P, Siniscalchi M (2003). Rationalization and Incomplete Information. Advances in Theoretical Economics 3(1): 1-46.

Baye MR, Morgan J (2004) Price dispersion in the lab and on the internet: theory and evidence. RAND Journal of Economics 35: 449-466.

Becker T, Carter M, Naeve J (2005). Experts playing the traveler's dilemma. Department of Economics working paper Nr. 252/2005, University of Hohenheim, Germany.

Bell D (1982). Regret in decision making under uncertainty. Operations Research, 30(5): 961-981.

Bell D (1985). Disappointment in decision making under uncertainty. Operations Research, 33(1): 1-27.

Bernheim D (1984). Rationalizable strategic behaviour. Econometrica 52(4): 1007-1028.

Binmore K (1987). Modelling Rational Players. Economics and Philosophy 3: 179-214.

Börgers T (1993). Pure Strategy Dominance. Econometrica, 61(2): 423-430. 
Brandenburger A (2003). On the existence of a "complete" possibility structure. In: Basili M, Dimitri N, Gilboa I (Eds.), Cognitive Processes and Economic Behavior. Routledge, pp. 30-34. Brandenburger A, Dekel E (1987). Rationalizability and Correlated Equilibria. Econometrica 55(6): 1391-1402.

Cubitt R. P, Sugden R (2011). The reasoning-based expected utility procedure. Games and Economic Behavior, 71(2): 328-338.

Dato S,Grunewald A, Müller D (2015). Expectation-Based Loss Aversion and Strategic Interaction (Discussion Paper 02/2015). Bonn Econ Discussion Papers.

Dekel E, Fudenberg D, Morris S (2006). Topologies on Types. Theoretical Economics 1: 275-309.

Duggan J, Le Breton M (2014). Choice-theoretic Solutions for Strategic Form Games (Working Paper No. 580). Rochester Center for Economic Research.

Fey M, McKelvey R, Palfrey T (1996). An Experimental Study of Constant-Sum Centipede Game. International Journal of Game Theory. 25: 269-287.

Friedenberg A (2010). When do type structures contain all hierarchies of beliefs? Games and Economic Behavior 68: 108-129.

Friedenberg A, Keisler HJ (2011). Iterated dominance revisited. In: Proceedings of the behavioral and quantitative game theory: Conference on future directions. ACM, New York, NY.

Gill D, Stone R (2010). Fairness and Desert in Tournaments. Games and Economic Behavior, 69: 346-364.

Guy F (1991). A Theory of Disappointment Aversion. Econometrica, 59(3): 667-686

Harsanyi J.C. (1967-68). Games with Incomplete Information Played by Bayesian Players, parts I-III. Management Science, 14: 159-182, 320-334 and 486-502.

Heifetz A, Samet D (1998). Topology-Free Typology of Beliefs. Journal of Economic Theory 82: $324-341$.

Heukelom F (2014). Behavioral Economics: A History, 1st edn. Cambridge University Press, Cambridge.

Hu T (2007). On p-Rationalizability and Approximate Common Certainty of Rationality. Journal of economic Theory 136: 379-391.

Kahneman D (2011). Thinking Fast and Slow, 1st edn. Allen Lane. 
Kahneman D, Tversky A (1979). Prospect Theory: An Analysis of Decision under Risk. Econometrica, 47(2): 263-292.

Kőszegi B, Rabin M (2006). A Model of Reference-Dependent Preferences. Quarterly Journal of Economics, 121(4): 1133-1165.

Kőszegi B, Rabin M (2007). Reference-dependent risk attitudes. American Economic Review, 97(4): 1047-1073.

Lewis D. K. (1969). Convention. Harvard University Press, Cambridge.

Llerasy JS, Piermontz E, Svobodax R (2015). Reference Dependence and Attitudes towards Uncertainty.

Loomes G, Sugden R (1982). Regret theory: An alternative theory of rational choice under uncertainty. The Economic Journal, 92(368): 805-824.

Loomes G, Sugden R (1986). Disappointment and dynamic consistency in choice under uncertainty. The Review of Economic Studies, 53(2): 271-282.

McKelvey R, Palfrey T (1992). An Experimental Study of the Centipede Game. Econometrica. 60: $803-836$.

McKelvey R, Palfrey T (1995). Quantal Response Equilibria for Normal Form Games. Games and Economic Behaviour 10: 6-38.

March JH, Simon H (1958). Organizations. John Wiley and Sons.

Masatlioglu Y, Raymond C (forthcoming). A Behavioral Analysis of Stochastic Reference Dependence. American Economic Review.

Moderer D, Samet D (1989). Approximating Common Knowledge with Common Beliefs. Games and Economic Behavior 1: 170-190.

Mounir A, Perea A, Tsakas E (2013). Common Belief in Approximate Rationality. Mimeo. Maastricht University.

Nagel R (1995). Unravelling in guessing games: an experimental study. American Economic Review 85: 1313-1326.

Pacuit E, Roy O (2016) Epistemic Foundations of Game Theory, The Stanford Encyclopedia of Philosophy (Spring 2016 Edition), Edward N. Zalta (ed.).

Pearce D (1984). Rationalizable strategic behaviour and the problem of perfection.Econometrica 52(4):1029-1050. 
Perea A (2012). Epistemic Game Theory: Reasoning and Choice, 1st edn. Cambridge University Press, Cambridge.

Perea A (2014). From Classical to Epistemic Game Theory. International Game Theory Review, 16(1): 1-22.

Perea A (2015). Finite Reasoning Procedures for Dynamic Games. In: Van Benthem, J., Ghosh, S. and Verbrugge, R. (eds.), Models of Strategic Reasoning: Logics, Games and Communities. Heidelberg: Springer.

Perea A, Roy S (2014). A New Epistemic Characterization of Proper Rationalizability. Mimeo.

Radner R (1980). Collusive Behaviour in Non-Cooperative Epsilon Equilibria of Oligopolies with Long but Finite Lives. Journal of Economic Theory 22:121-157.

Radner R (1981). Monitoring Cooperative Agreements in a Repeated Principal-Agent Relationship. Econometrica 49(5), 1127-1148.

Rosenthal R (1981). Games of Perfect Information, Predatory Pricing and the Chain-Store Paradox. Journal of Economic Theory. 25: 92-100.

Rosenthal R (1989). A Bounded-Rationality Approach to the Study of Noncooperative Games. International Journal of Game Theory 18: 273-292.

Rubinstein A (1991). Comments on the interpretation of game theory. Econometrica. 59: 909-924.

Shalev J (2000). Loss Aversion Equilibrium. International Journal of Game Theory 29: 269-287.

Shimoji M, Watson J (1988). Conditional Dominance, Rationalizability and Game Forms. Journal of Economic Theory, 83: 161-195.

Sugden R (2003). Reference-Dependent Subjective Expected Utility. Journal of Economic Theory 111: 172-191.

Tan T, Werlang S (1988) The Bayesian foundations of solution concepts of games. Journal of Economic Theory 45:370-391.

Tversky A, Kahneman D (1992). Advances in Prospect Theory: Cumulative Representation of Uncertainty. Journal of Risk and Uncertainty 5: 297-323. 


\section{Valorisation}

"Knowledge valorization refers to the process of creating value from knowledge, by making knowledge suitable and/or available for social (and/or economic) use and by making knowledge suitable for translation into competitive products, services, processes and new commercial activities."

— adapted definition based on the National Valorization Committee 2011:8

Valorisation as defined by the National Valorization Committee is the process by which knowledge created by research is made available to society and by which it is transformed into economic and social impact. Such impact can take many forms. Although most commonly thought of in terms of new products, services and processes, research impact can also take the form of scientific publications serving as basis for further research or a tool to spark public debate about certain phenomenon of societal or economic relevance. The form the impact of any research depends on the nature of such research, with a broad distinction made between fundamental and applied research.

Fundamental research seeks to develop and accumulate knowledge for the sake of the knowledge itself. Focus is thus often on exploring an abstract, general or philosophical question. Findings often also take the form of generalizations abstracting away from any specific real life practical applications that might have motivated the question to begin with. Applied research, on the other hand, focusses on answering a specific practical question, usually by developing new products and processes. Given the nature of applied research, it is mostly straightforward to think of its mediums of impact. Valorisation opportunities in fundamental research, on the other hand, are often achieved indirectly and over an extended period of time. It is fundamental research that uncovers links between phenomena previously thought of as unlinked and forms the basis upon which future applied research is based.

The field of epistemic game theory to which this thesis belongs is concerned with the reasoning process of individuals in situations of strategic interaction and the choice behavior resulting therefrom. This thesis investigates abstract epistemic models of reasoning of individuals attempting to make the "best possible" choices given the strategic uncertainties they face. This 
thesis therefore belongs to the category of fundamental research. In what follows, potential future steps towards the realization of the economic and societal impact of the models at hand are discussed.

A characterizing aspect of epistemic game theory is the focus on players beliefs about their opponents' choices, about any unknown parameters in the game and about their opponents' beliefs. Hence, epistemic game theory makes explicit the fact that choice behavior of a player in a game does not only depend on that player's preferences but also on his beliefs. This is inline with a growing stream in the experimental literature attempting to investigate the role players' beliefs fulfill in a given strategic situation through belief elicitation. Belief elicitation experiments try to make both the choices and the beliefs of players in a given game observable. Epistemic models such as the ones developed in this thesis can thus serve as a source for experimentally testable hypotheses and as a tool to formalize observed results in experiments.

Models investigated in this thesis examine deviations of individuals' choices from patterns prescribed by perfect rationality models, i.e. models where players are assumed to maximize subjective expected utility given their beliefs about their opponents' choices and beliefs and about any other unknown parameters of the game. These deviations might find their origins in the cognitive limitations of individuals or in the interference of emotions such as regret, rejoice or spite. In any case, various forms of bounded rationality have been used to explain deviations of players' choice behavior from choices prescribed by perfect rationality models both inside and outside the laboratory. Plentiful evidence supporting a significant role of fairness and reciprocity considerations has been found in laboratory experiments.

However, the role of bounded rationality models in explaining behavior has not been confined to experiments. One form of bounded rationality assumes players to be reference dependent rational, where players evaluate every choice relative to some reference point. The latter could be anything from the status quo level of wealth to a choice the player has made in similar or identical game or even a belief the player holds about his future choice and that of his opponent. Facing reference-dependent rational buyers, a seller on an online second-price sealed-bid auction is shown to be better off augmenting his auction with a Buy It Now option along with his reservation price, a phenomenon often witnessed nowadays in eBay auctions for example. Moreover, models of bounded rationality play an important role in the field of marketing where firms account for possible behavioral anomalies on the consumers part in setting their prices and designing their advertising strategies.

Like many reasoning concepts in the field of epistemic game theory, along with each of the models introduced in this thesis a characterizing computational procedure is defined. Thus in 
addition to possible experimental applications of our models to uncover players' beliefs, the mere characterization of choices players could reasonably make under a give epistemic concept may help gain more insights in the actual prominence of the different reasoning models players follow in reality. It is worth noting that testable hypotheses provided by epistemic game theory models in such case are different in nature from those obtained from classical game theory. The latter is focused on identifying the equilibrium outcome of a game which dictates a profile of strategies for players and implicitly assumes all players hold correct beliefs about their opponents's profiles of choices and beliefs. Since it is highly unlikely that all players would hold such correct beliefs, such assumption can be fairly judged as unrealistic except may be for situations in which players interact repeatedly with the same opponents.

Epistemic concepts such as the ones developed in this thesis drop that correct belief assumption. If accompanied by an algorithm characterizing those choices that players can be expected to make under the respective reasoning concept (like the model of chapter 4), the characterized set of choices for any player in any given game is going to be a superset of the set of choices consistent with the corresponding equilibrium concept, if one exists. Those sets of choices can provide more reasonable testable hypotheses in games where players often deviate from the perfect rationality outcome in practice. One example for which this has been shown is experimental evidence on first-price sealed-bid auctions.

Theoretical predictions often put to the test in experiments of first-price auctions are based on the risk neutral Nash equilibrium where players are assumed to be perfectly rational and are assumed to have correct beliefs about their opponents' choices and beliefs. Experimental data however indicates systematic deviations from theoretical equilibrium predictions in first-price sealed-bid auctions often in the form of overbidding. Relaxing the correct beliefs assumption it can be shown that a certain range of bids above the equilibrium choice can be reasonably expected to be made by a rational player believing in his opponents' rationality, believing in his opponents' belief in their opponents' rationality and so on.

Hence, potential extensions and applications of the type of models introduced in this thesis mainly take the form of future research, often within the experimental lab. The findings of the latter could in turn be translated into research applied to real life strategic situations. Nevertheless, general intuitions and conclusions derived from theoretical models can in some cases be used to formulate testable hypotheses for real life games directly. Therefore, the most important medium of knowledge valorisation in the case of this thesis would be scientific publications. Publishing theoretical models such as ours in high impact journals widely read in the scientific and academic world would facilitate follow-up theoretical as well as empirical research. 
It is worth noting that models in epistemic game theory often draw their motivation from empirically observed behavior inside and outside the experimental lab. Doing so, these models seek to generalize such observed behavior in theoretical models abstracting away from the specifics of the motivating cases to allow the intuition to extend to distinct yet similar situations. Hence, theoretical models themselves serve as an indirect valorisation channel of empirical research by generalizing the findings of the latter and paving the way for further steps of empirical exploration of human behavior in strategic situations. Although perfectly evident in the academic community, the fact that such theoretical models are so closely tied to their empirical counterparts is something that is often not that clear in the public eye.

Therefore, other forms of publications such as newspaper articles or blogs could play a vital role in bringing theoretical insights and its links to empirical findings into the public eye. More accessible publications could also spark public debate about potential applications of theoretical models. In addition to bringing theoretical models closer to the needs and questions of society, such debate could in turn feed the applied research community with an interesting source of further research questions. However most importantly, this might help raise awareness of the complexity of decision making in numerous strategic situations encountered in daily social and economic interactions between individuals. 


\section{Nederlandse Samenvatting}

Speltheorie bestudeert strategische interactie tussen spelers waar de nut van een speler niet alleen afhankelijk is van zijn eigen keuze maar ook van die van zijn tegenstanders. In dergelijke situaties is het aannemelijk te veronderstellen dat spelers een redeneringsproces volgen, met als doel om de best mogelijke keuzes te bepalen gegeven de onzekerheid. Binnen de epistemische speltheorie staat zo'n rederneringsproces centraal. Onzekerheid in een spel kan betrekking hebben op ieder aspect van de omgeving waarin de spelers zich bevinden. Het basis domein van onzekerheid van een speler bevat naast de keuzes van zijn tegenstanders ieder parameter van het model dat voor de speler onbekend is. Naast het basis domein van onzekerheid wordt de speler ook geconfronteerd met onzekerheden rondom de overtuigingen van zijn tegenstander(s) over hun respectievelijke basis domeinen van onzekerheid, de overtuigingen van de tegenstanders over de overtuigingen van hun respectievelijke tegenstanders, enzovoort. Het redeneringsproces omvat dus de vorming van de speler's overtuigingen over zijn basis onzekerheid, overtuigingen over de overtuigingen van tegenstanders over hun basis onzekerheden, enzovoort.

Het proces van redeneren over het betreffende spel en de overtuigingen van de tegenstanders resulteert in de vorming van hiërarchieën van overtuigingen of "belief hierarchies". Bestaande uit een oneindig aantal lagen, is een belief hierarchy een cruciaal maar ook zeer complex object. Een manier om deze belief hierarchies te codificeren is door gebruik te maken van een "type space model" (Harsanyi 1967-68). Hoewel het model eerst ontwikkeld werd door Harsanyi om de overtuigingen van spelers in spellen van onvolledige informatie te coderen en het spel vervolgens te transformeren in een spel van onvolmaakte informatie, werd het later gebruikt om de overtuigingen van spelers over elke vorm van interactieve onzekerheid in een spel te modelleren. In een type space model, krijgt iedere speler een verzameling van typen toegewezen, elk type induceert een mogelijke belief hierarchy voor de betreffende speler. Bij ieder epistemisch type van een speler behoort een overtuigen over de verschillende combinaties van basis onzekerheid en typen van tegenstanders.

Beperkingen opgelegd aan belief hierarchies in epistemische concepten vertrekken meestal van de aanname dat spelers op een bepaalde manier redeneren of een bepaalde keuzeregel hanteren, dat spelers geloven dat hun tegenstanders op dezelfde manier redeneren en keuzes maken, enzovoorts. Volgens Common Belief in Rationality (CBR) gelooft iedere speler dat zijn tegenstanders rationeel zijn, dat zijn tegenstanders geloven dat hun tegenstanders rationeel zijn, 
enzovoorts. CBR vertrekt vanuit de aanname dat spelers onbegrensd rationeel zijn en is het concept waarmee andere epsitemische concepten in de literatuur worden vergeleken. Aangezien dit proefschrift over begrensde rationaliteit gaat, zal CBR hier ook als "benchmark" worden gebruikt.

Dit proefschrift neemt twee vormen van begrensde rationaliteit onder beschouwing in een epistemisch kader. In hoofdstukken 2 en 3 streven spelers om het verwachte nut te maximaliseren maar kennen ze tegelijk een positieve kans toe aan de mogelijkheid dat de tegenstanders fouten maken en/ of dat de tegenstanders vergissingen van hun respectievelijke tegenstanders mogelijk achten. Gezien spelers zo dicht mogelijk bij de rationele nut-maximaliserende keuze willen komen zeggen we dat spelers "bijna rationeel" (approximately rational) handelen. Hoofdstuk 2 definieert Common Belief in Approximate Rationality voor statische spellen en hoofdstuk 3 breid het model uit naar dynamische spellen. Hoofdstuk 2 laat zien dat het toekennen van een relatief kleine kans aan mogelijke vergissingen van tegenstanders theoretische voorspellingen in de beroemde Traveler's Dilemma veel dichter bij experimenteel waargenomen resultaten brengt.

In hoofdstuk 4 is het redeneringsproces en dus de keuzes van de spelers context afhankelijk. Het verwachte nut van een keuze hangt in zo'n geval af van de mate waarin die keuze afwijkt van een referentiepunt. Verder draagt het voorkomen van een potentieel verlies meer bij aan het verwachte nut dan een potentiële winst van dezelfde omvang. Referentiepunten komen in verschillende vormen voor in de literatuur. Een referentiepunt kan bijvoorbeeld een huidig of voormalig bezit zijn. Ook kan het een eerder gemaakte keuze in een identiek of vergelijkbaar spel zijn en wordt het in zo'n geval een referentiekeuze genoemd. Het referentiepunt van een speler in hoofdstuk 4 is een combinatie van een referentiekeuze en een overtuiging over zijn basis domein van onzekerheid. Hoewel het model niet verder ingaat op de wijze waarop een referentiepunt tot stand komt, is het model wel van voldoende flexibiliteit om verschillende definities van het referentiepunt toe te staan. Het hoofdstuk introduceert het epistemisch concept van Common Belief in Reference-Dependent Rationality waar het referentiepunt van een speler alleen voor hem bekend is. In ieder hoofdstuk wordt een rekenkundige procedure geïntroduceerd om het epistemisch model van het hoofdstuk te karakteriseren. Iedere procedure bepaalt voor een gegeven spel welke keuzes spelers kunnen maken indien ze volgens het desbetreffende model redeneren. 


\section{Curriculum Vitae}

Angie Mounir was born on September 11th, 1984 in Alkmaar, the Netherlands. In 2005, she obtained a bachelor's degree in economics and statistics at Cairo University. She started a master's degree in economics at Cairo University with a focus on industrial organization and experimental economics. Between 2006 and 2010 she was a teaching assistant at Cairo University and worked part-time as a researcher on diverse consultancy projects. In 2010 she obtained a master's degree in mathematical economics from Maastricht University. In 2011 she became a PhD student in the department of quantitative economics of Maastricht University in the filed of epistemic game theory under the supervision of Andrés Perea and Elias Tsakas. 